\title{
Assessment of Slat Noise Predictions for 30P30N High- Lift Configuration from BANC-III Workshop
}

\author{
Meelan Choudhari ${ }^{1}$ and David P. Lockard ${ }^{2}$ \\ NASA Langley Research Center, Hampton, VA 23681 \\ and the BANC-III Category-7 Team ${ }^{3}$
}

This paper presents a summary of the computational predictions and measurement data contributed to Category 7 of the $3^{\text {rd }}$ AIAA Workshop on Benchmark Problems for Airframe Noise Computations (BANC-III), which was held in Atlanta, GA, on June 14-15, 2014. Category 7 represents the first slat-noise configuration to be investigated under the BANC series of workshops, namely, the 30P30N two-dimensional high-lift model (with a slat contour that was slightly modified to enable unsteady pressure measurements) at an angle of attack that is relevant to approach conditions. Originally developed for a CFD challenge workshop to assess computational fluid dynamics techniques for steady high-lift predictions, the 30P30N configurations has provided a valuable opportunity for the airframe noise community to collectively assess and advance the computational and experimental techniques for slat noise. The contributed solutions are compared with each other as well as with the initial measurements that became available just prior to the BANC-III Workshop. Specific features of a number of computational solutions on the finer grids compare reasonably well with the initial measurements from FSU and JAXA facilities and/or with each other. However, no single solution (or a subset of solutions) could be identified as clearly superior to the remaining solutions. Grid sensitivity studies presented by multiple BANC-III participants demonstrated a relatively consistent trend of reduced surface pressure fluctuations, higher levels of turbulent kinetic energy in the flow, and lower levels of both narrow band peaks and the broadband component of unsteady pressure spectra in the nearfield and farfield. The lessons learned from the BANC-III contributions have been used to identify improvements to the problem statement for future Category-7 investigations.

\section{Nomenclature}

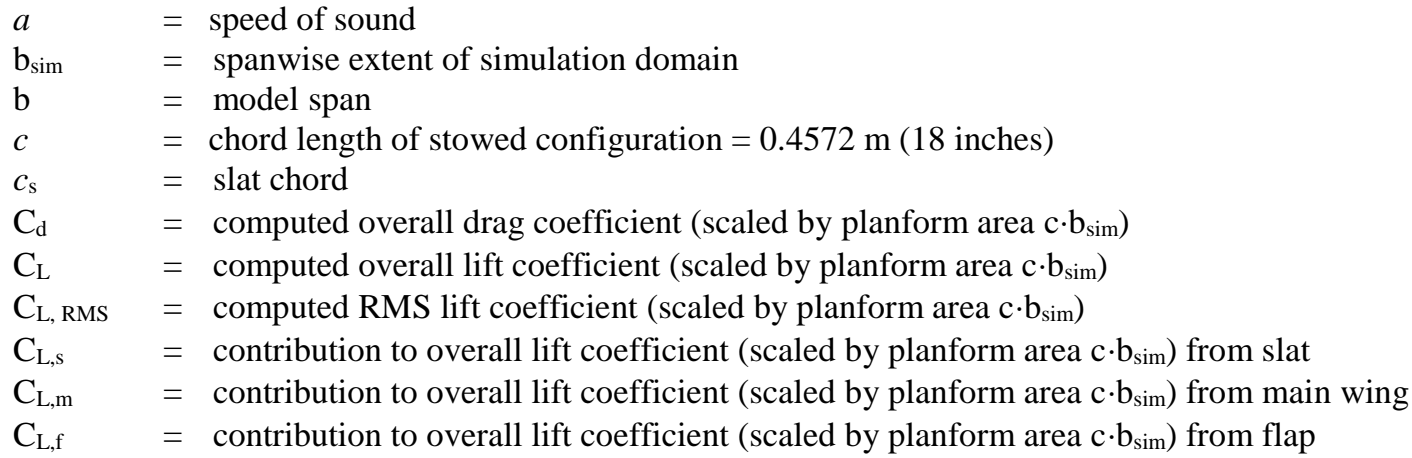

\footnotetext{
${ }^{1}$ Aerospace Technologist, Computational AeroSciences Branch, M.S. 128. Associate Fellow, AIAA. ${ }^{2}$ Aerospace Technologist, Computational AeroSciences Branch, M.S. 128. Senior Member, AIAA.

${ }^{3}$ Team members are listed in Table 1.
} 


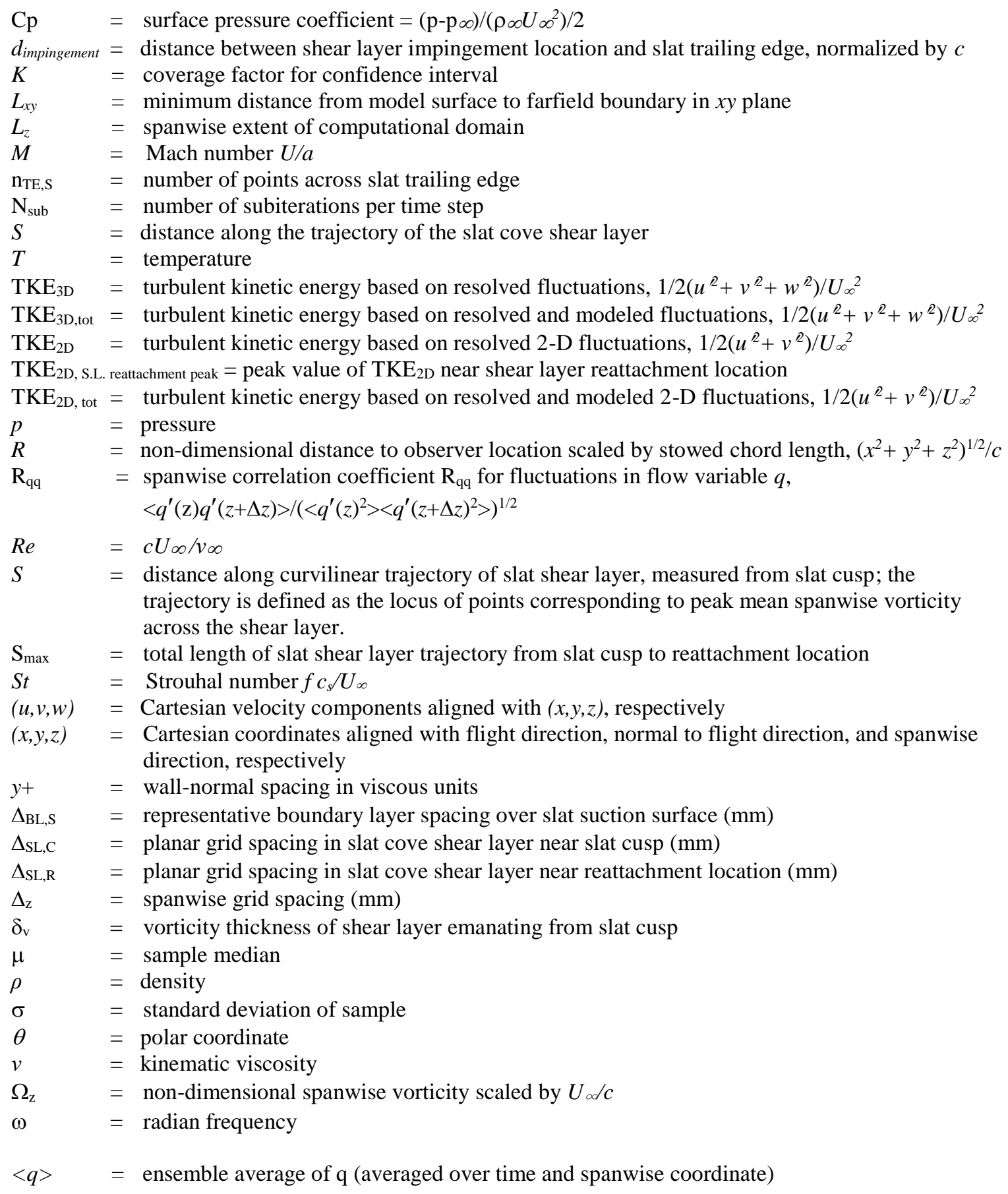

\section{Subscripts}

$w \quad=$ wall

$\infty \quad=$ freestream

Superscripts

$=$ instantaneous fluctuation about the mean

Prefixes

$\Delta \quad=$ grid spacing or separation along specified coordinate direction 


\begin{tabular}{|c|c|c|}
\hline & & $\begin{array}{c}\text { Abbreviations } \\
\end{array}$ \\
\hline AIAA & $=$ & American Institute of Aeronautics and Astronautics \\
\hline AMR & $=$ & Adaptive Mesh Refinement \\
\hline AoA & $=$ & angle of attack \\
\hline $\mathrm{ARC}$ & $=$ & Ames Research Center \\
\hline BANC & $=$ & Benchmark Problems for Airframe Noise Computations \\
\hline $\mathrm{BC}$ & $=$ & boundary condition \\
\hline CAA & $=$ & Computational Aeroacoustics \\
\hline CFD & $=$ & Computational Fluid Dynamics \\
\hline CTR & $=$ & Center for Turbulence Research \\
\hline DDES & $=$ & Delayed Detached Eddy Simulation \\
\hline DES & $=$ & Detached Eddy Simulation \\
\hline F.E. & $=$ & fine equivalent (voxels) \\
\hline FRPM & $=$ & Fast Random Particle Method \\
\hline FSU & $=$ & Florida State University \\
\hline FWH & $=$ & Ffowcs-Williams and Hawkings \\
\hline LaRC & $=$ & Langley Research Center \\
\hline LBM & $=$ & Lattice Boltzmann Method \\
\hline IDDES & $=$ & Improved Delayed Detached Eddy Simulation \\
\hline ILES & $=$ & Implicit Large Eddy Simulation \\
\hline JAXA & $=$ & Japan Aerospace Exploration Agency \\
\hline $\mathrm{KTH}$ & $=$ & Royal Institute of Technology, Stockholm, Sweden \\
\hline LES & $=$ & Large Eddy Simulation \\
\hline NASA & $=$ & National Aeronautics and Space Administration \\
\hline N/A & $=$ & not applicable \\
\hline NBP & $=$ & Narrow Band Peak \\
\hline NLDE & $=$ & Nonlinear Disturbance Equations \\
\hline PDF & $=$ & probability density function \\
\hline PIV & $=$ & Particle Image Velocimetry \\
\hline Ppw & $=$ & points per wavelength \\
\hline PSD & $=$ & Power Spectral Density $(\mathrm{dB} / \mathrm{Hz})$ \\
\hline PSP & $=$ & Pressure Sensitive Paint \\
\hline QFF & $=$ & Quiet Flow Facility \\
\hline RANS & $=$ & Reynolds Averaged Navier Stokes \\
\hline RMS & $=$ & root mean square \\
\hline SA & $=$ & Spalart Allmaras \\
\hline SGS & $=$ & subgrid scale (stress) \\
\hline SST & $=$ & Shear Stress Transport \\
\hline SPIV & $=$ & Stereoscopic Particle Image Velocimetry \\
\hline VLES & $=$ & very large eddy simulation \\
\hline WMLES & $=$ & wall modeled large eddy simulation \\
\hline
\end{tabular}

\section{Introduction}

IRFRAME noise corresponds to the acoustic radiation due to turbulent flow in the vicinity of airframe components such as high-lift devices and landing gear. The combination of geometric complexity, high Reynolds number turbulence, multiple regions of separation, and a strong coupling with adjacent physical components makes the prediction and control of airframe noise highly challenging. Since 2010, the American Institute of Aeronautics and Astronautics (AIAA) has organized an ongoing series of workshops devoted to Benchmark Problems for Airframe Noise Computations (BANC). The BANC workshops target a selected set of canonical yet realistic configurations for enabling systematic progress in the understanding and high-fidelity predictions of airframe noise via collaborative investigations that integrate state of the art computational fluid dynamics, computational aeroacoustics, and in-depth, holistic, and multi-facility measurements. A total of eight problem categories have been addressed thus far and the BANC-III 
Workshop $^{1}$ in Atlanta, GA, on June 14-15, 2014, focused on categories 1 (airfoil trailing edge), 4 (partiallydressed, cavity-closed nose landing gear), 5 (ONERA/Airbus LAGOON Simplified Landing Gear configuration), 6 (slat noise - DLR F15 and F16 configuration), 7 (slat noise - modified 30P30N configuration), and 8 (propagation phase of airframe noise prediction).

The BANC series represents the second set of workshops organized by AIAA following the success of the Drag Prediction Workshops ${ }^{2-9}$ and is the first workshop to be co-sponsored by the Aeroacoustics and Fluid Dynamics Technical Committees of AIAA. A brief overview of the BANC workshops is given in Ref. 10. The highlights of the BANC workshops include: a focus on unsteady flows, a strong emphasis on advancing both computational and measurement techniques, and a long-term, collective focus on selected canonical problems across multiple workshops. The broad technical objectives of these workshops are as follows:

1. Provide a forum for a thorough assessment of simulation-based noise-prediction tools in the context of airframe configurations including both nearfield unsteady flow and the acoustic radiation generated by the interaction of this flow with solid surfaces.

2. Identify current gaps in physical understanding, experimental databases, and prediction capability for the major sources of airframe noise.

3. Help determine best practices, and accelerate the development of benchmark quality datasets.

4. Promote future coordinated studies of common configurations for maximum impact on the current state of the art in the understanding and prediction of airframe noise.

For an overview of the accomplishments of the BANC-I and BANC-II workshops, the reader is referred to the proceedings of those workshops ${ }^{11,12}$ as well as the summary papers for problem categories related to trailing edge noise, ${ }^{13}$ unsteady wake interference between a pair of in-line tandem cylinders, ${ }^{14}$ the Rudimentary Landing Gear ${ }^{15,16}$ and the PDCC-NLG Landing Gear. ${ }^{17}$ Category 7 of the BANC series of workshops targets the slat cove noise associated with the generic, zero sweep, 30P30N high-lift configuration. ${ }^{18}$ For the BANC-III Workshop, a total of twelve teams performed computational predictions of various aerodynamic and aeroacoustic metrics ranging from mean and unsteady force coefficients, surface pressure distributions including power spectral density (PSD) and spanwise coherence, off-body velocity field in regions of attached and separated flow, and acoustic spectra representative of flyover measurements. Three other teams contributed complementary information derived from previously conducted simulations for the same geometry and either the same or related flow conditions. Measurements were performed in wind tunnel facilities at Florida State University ${ }^{19}$ in the USA and at JAXA ${ }^{20}$ in Japan. The nominal flow conditions of interest involved a Mach number of 0.17 , Reynolds number based on stowed chord of 1.71 million, and an equivalent free-air angle of attack (AoA) equal to 5.5 degrees.

This paper provides a summary of the Category 7 contributions to the BANC-III workshop with the objective of documenting the current state-of-the-art in the prediction and measurement of slat-cove aeroacoustics, so as to provide a reference for tracking future progress and to identify specific areas where improvements are needed. Summaries of Category 5 (LAGOON landing gear) and Category 8 (propagation phase of airframe noise prediction) contributions at the BANC-III Workshop are given in the accompanying papers by Manoha et al. ${ }^{21}$ and Lopes et al., ${ }^{22}$ respectively. An outline of the remaining paper is as follows. Section II provides a brief description of the Category 7 problem statement. An overview of the various contributions is provided in Section III, followed by a comparison of the computational solutions with each other and, also with the measurements, in Section IV. A summary is provided in Section V.

\section{Case Description}

This section describes the significance of problem Category 7 from the BANC-III Workshop, outlines the specific technical objectives behind this category, and provides a brief description of the canonical flow configuration selected for this category.

\section{A. Problem Significance}

Noise radiation from the leading-edge slat of a high-lift system is known to be an important component of aircraft noise during approach. ${ }^{23}$ Slat noise is primarily broadband, but may be accompanied by multiple narrowband, tonal peaks (NBPs) that also occur within the frequency range of highest broadband noise. The occurrence and the relative strength of the NBPs depends on several factors including the geometry of the configuration and the flow conditions. Problem Category 7 from the BANC series of workshops targets slat noise in the most rudimentary approach setting of a generic, unswept, 3-element, high-lift configuration. A 
validated prediction capability and the understanding of noise source mechanisms for this simplified configuration will provide a strong basis for addressing the complexities of slat noise in the context of a realistic high-lift configuration, e.g., sweep, taper, twist, brackets, and geometric details of an operational slat. Numerical simulations of slat noise involve a number of challenges; these include a high computational cost associated with large spanwise domains (which may be needed in spite of the quasi-2-D behavior of a high-lift configuration of large aspect ratio) and the physical complexity of the flow field (which makes it difficult to precisely identify the noise generation mechanisms). Therefore, a holistic and team oriented approach focused on a simple configuration is the most effective way to advance the computational state-ofthe-art for this class of problems.

\section{B. Objectives}

Slat noise is a complex aeroacoustic problem with several possible noise generation mechanisms (Fig. 1) that have not been fully identified, understood, or ranked in their relative importance. The specific technical objectives of the problem Category 7 are:

1. To enable a multi-faceted assessment of high-fidelity slat noise simulations via comparisons with nearfield (i.e., aerodynamic) and acoustic measurements,

2. To use the simulation database for collective advancement of the understanding of slat noise sources,

3. To improve the experimental methodology for aeroacoustic testing of high-lift devices and identify future measurements that would facilitate the accomplishment of the previous two goals; and

4. To determine the best practices for aeroacoustic simulations of slat noise, analysis of computational data, and comparison with wind tunnel experiments.

In summary, the goal is to develop a substantially revised version of Fig. 1 that is based on a thorough analysis of both simulations and measurements and reflects the consensus view of the airframe noise community. These objectives differ in scope from those of the High-Lift Prediction Workshops, ${ }^{24,25}$ which are focused primarily on mean loading characteristics of high-lift configurations over a broad range of angles of attack. Yet, because the mean aerodynamics is coupled with the development of unsteady flow structures contributing to noise generation, the lessons learned from the High-Lift Prediction Workshops should also be of benefit to the Category 7 participants.

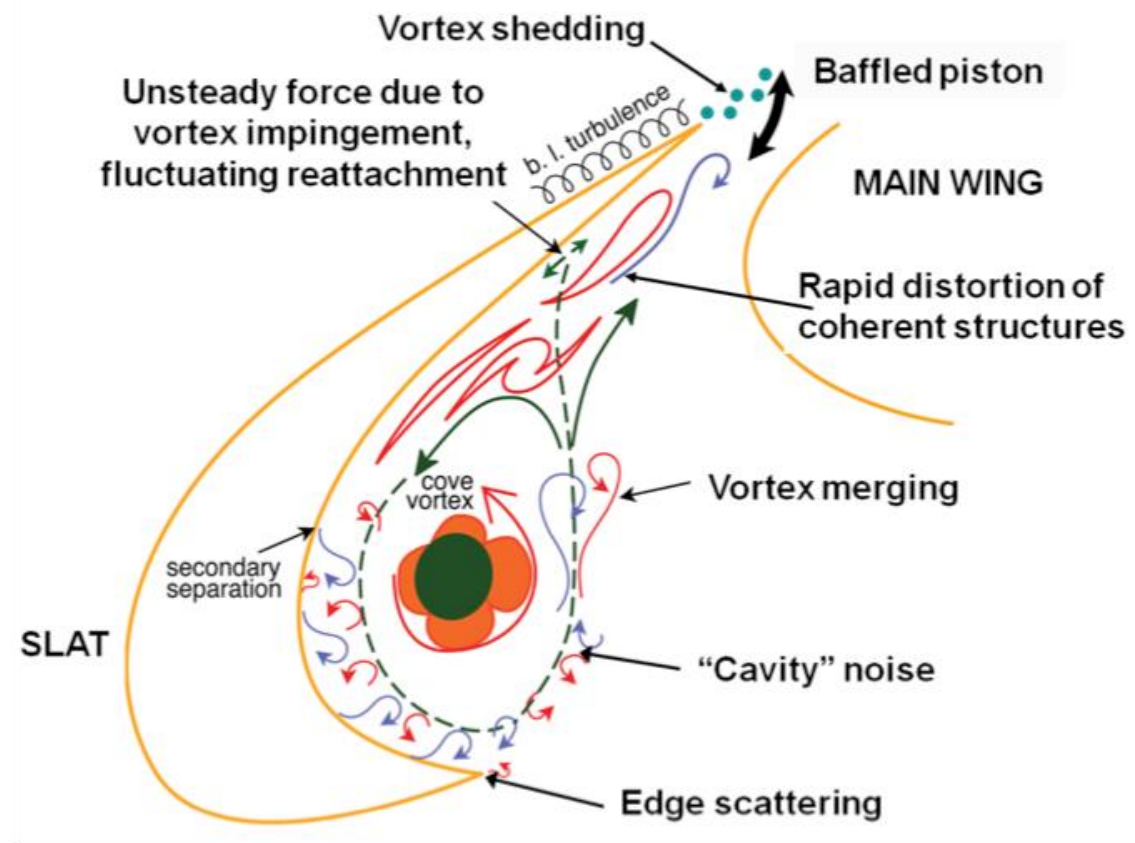

Figure 1. Suspected slat noise sources 


\section{Physical Configuration}

The generic, unswept, 3-element, high-lift configuration has been the focus of multiple wind tunnel and computational investigations at NASA Langley Research Center (LaRC) and elsewhere. The well-known 30P30N configuration (Fig. 2), designed by McDonnell-Douglas (now Boeing), corresponds to slat and flap deflections of $30^{\circ}$ each. ${ }^{26}$ The slat chord and flap chord of the model are approximately equal to 15 and 30 percent, respectively, of the stowed chord of $0.457 \mathrm{~m}$. At the baseline Mach number of $\mathrm{M}=0.17$ for the measurements using this configuration for airframe noise investigations, the Reynolds number, $R e$, based on the stowed chord length, $c$, equals $1.7 \times 10^{6}$.

The 30P30N high-lift configuration was first used for a High-Lift CFD Challenge Workshop aimed at 2-D, steady (or time-averaged) flow computations for high-lift airfoils. ${ }^{26}$ Subsequent measurements ${ }^{27,28}$ and computations $^{29-36}$ at NASA Langley Research Center targeted the unsteady aspects of the flow field associated with the 30P30N configuration. Two different wind tunnel entries in NASA's Basic Aerodynamic Research Tunnel (BART) provided particle image velocimetry (PIV) measurements of the unsteady flow field within the slat cove region, along with steady pressure measurements on the entire airfoil. Both planar and stereo PIV measurements were obtained to characterize the planar and cross-plane structures within this flow.

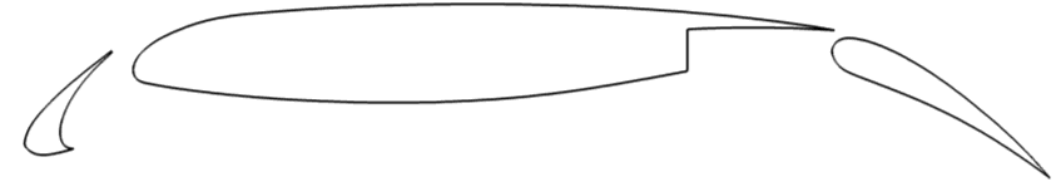

Figure 2. Cross-sectional view of the 3-element 30P30N high-lift configuration.

The slat geometry for the BANC series of workshops involves minor modifications to the slat contour from the original 30P30N configuration used for the CFD Challenge Workshop ${ }^{26}$ (as well as a more recent AIAA Workshop targeting high-order CFD methods ${ }^{37,38}$ ) and also during the nearfield measurements in the BART facility. ${ }^{27}$ These changes were made to allow the incorporation of unsteady pressure transducers close to the reattachment location along the pressure surface of the leading-edge slat. Steady RANS computations were performed to ensure that the effect of these modifications was small. Participants in the BANC-III Workshop were asked to perform computations for a flight, i.e., free-air, configuration at 5.5-deg angle of attack. The choice of a free-air configuration was intended to allow acoustic predictions without interference from wind tunnel walls, while simultaneously approximating the previously available measurements in the BART facility at 4-deg AoA. In return, however, one must accept the resulting challenges in comparing computational data with wind tunnel measurements, since the aerodynamics of high-lift configurations in test sections of modest size (the kind that is typically available for the in-depth fundamental measurements targeted in the BANC workshops) is significantly influenced by the presence of tunnel walls. ${ }^{19,20,39}$ The requirements of a benchmark dataset will not be achieved in all cases, especially in a single round of workshops, and hence, the title of the BANC series reflects the quest for the benchmark datasets and the collective journey towards that goal.

Measurements of surface pressure fluctuations and acoustic radiation were made for the BANC-III workshop ${ }^{19,20}$ as described in Section III of this paper, with plans to acquire more in-depth data in follow-on tests. The future measurements will also address the specific needs identified during BANC-III, with an emphasis on quantifying the robustness of the experimental findings and assessing the uncertainties via measurements of the same configuration in different facilities.

\section{Simplifications and Challenges}

The present choice of flow conditions and geometric configuration for the slat noise component of the BANC-III Workshop has both its advantages and limitations. The lack of a universal benchmark is the reason why the BANC workshops have sought to use more than one configuration for each dominant source of airframe noise. The 2-D geometry of the $30 \mathrm{P} 30 \mathrm{~N}$ configuration is simple enough to allow a broad set of flow solvers to be applied, which is confirmed by that the previous observation that this configuration has been embraced in a variety of workshops with rather different objectives. ${ }^{18,26,37}$ The low Reynolds number 
in Category 7 of the BANC-III workshop also implies that boundary layer transition over the slat should not be a major influence on the overall computation because the flow over the majority of the slat is laminar at this Re. Separation points on the slat are fixed by the model geometry; however, the reattachment location is jointly determined by the overall surface pressure distribution and the accuracy in predicting the evolution of unsteady flow structures along the shear layer that bounds the separated flow region within the slat cove. At the angle of attack and Reynolds number of interest in Category 7, the flap boundary layer is expected to separate, and this separation location (the computational prediction of which will be depend on the turbulence model and the accuracy in predicting the overall loading characteristics), will also have an indirect effect on the pressure gradient imposed on the slat.

The 2-D geometry of the 30P30N configuration enables gridding simplifications as well as computational savings when used with periodic boundary conditions in span; however, such quasi-2-D configurations are not without their own set of challenges as demonstrated by the Tandem Cylinder configuration used in Category 2 of the BANC series of Workshops. ${ }^{10}$ As discussed in Ref. 12, the tandem cylinder category demonstrated the intricate effects of boundary layer tripping on the rear cylinder in spite of being buffeted by the strong unsteady wake from the front cylinder and also emphasized the effects of model installation within a wind tunnel facility as well as other facility details. The Category 2 investigations also indicated that, in spite of the relatively long span of the cylinder models (nearly 16 times the cylinder diameter), the decay in spanwise correlations was impacted by the presence of the side walls wherein the cylinders were mounted and, furthermore, that including the signature of unsteady flow events over the side plate surfaces accounted for a measurable correction to the farfield acoustics. ${ }^{40}$ Accounting for the model installation effects enabled a close match with the measured acoustics, including both the tonal peaks associated with vortex shedding and the broadband portion and, hence, also provided a meaningful basis to assess the computations without any installation effects, i.e., with spanwise periodic boundary conditions. The above issues are also expected to be relevant to the high-lift configuration of Category 7.

The integration of computational and measurement activities for the tandem cylinder configuration was primarily sequential in nature. ${ }^{12}$ Because of the relative simplicity of the configuration and a reasonable body of prior results from the literature (albeit at low Reynolds number and purely aerodynamic in nature), with due planning, a thorough set of measurements was acquired without a great deal of a priori assistance from numerical computations. However, as discussed by Choudhari and Yamamoto, ${ }^{12}$ the difficulties encountered during the tandem cylinder investigations are amplified in Category 7 . The factors contributing to the extra difficulties include: (i) the increased complexity in the noise generation (Fig. 1) resulting in multiple NBPs superimposed on the otherwise broadband spectrum of slat cove noise, (ii) large time averaged lift on the model which leads to large deflections of the tunnel stream in an open jet facility and, hence, leads to unacceptable variations in the aerodynamic characteristics of the model, (iii) aerodynamic and aeroacoustic effects of brackets connecting the slat and flap elements to the main wing, (iv) extraneous noise sources within the model such as main element cove, main and flap trailing edges, (v) possible separation over the flap, and (vi) more complex sidewall interference effects on the high-lift configuration. As a result of these difficulties, the modus operandi for Category 7 has been different from Category 2, with a tighter and necessarily parallel coupling between CFD and experiments. Hence, a concomitant set of multiphase computational and experimental investigations has been necessary for Category 7 . Even at an early stage, ${ }^{30}$ the interplay between computations and measurements established the need to pay careful attention to the spatial resolution of global measurement techniques like particle image velocimetry, especially in high gradient regions such as the initial region of shear layer development behind the slat cusp. Measurements at multiple resolutions were necessary to better characterize the scale disparity among noise-relevant unsteady flow structures. One of the goals of the BANC-III edition of Category 7 was to uncover additional issues of this type to enable further improvements in both simulation and measurement capabilities.

\section{Overview of Contributions}

Category 7 generated a strong response during the BANC-III Workshop, with a total of 24 contributions from 17 teams as listed in Table 1 . The table shows only 21 entries out of the total 24 . The LAVA team at NASA Ames (Submissions 13 and 14 from Table 1) performed three separate computations, but opted to withhold a preliminary simulation based on a hybrid, DDES-ILES methodology. NASA Langley and Exa Corporation performed PowerFLOW ${ }^{\circledR}$ computations on three separate grids (coarse, medium, and fine). As discussed in subsection IV.L, the results based on the medium and fine grids were quite close to each other; and, hence we deemed it sufficient to use only the fine grid solution for a majority of the analysis presented 
in this paper. There were two separate experimental contributions based on wind tunnel measurements at Florida State University and JAXA, respectively. In view of the previously described difficulties in making meaningful measurements of high-lift aeroacoustics, the two experimental datasets are also viewed as contributions rather than as benchmarks for the computations. The activities of the Category 7 group should enable sufficient advances to elevate these datasets to the status of benchmarks. A brief description of each experiment is given below, followed by observations about the computational contributions. For additional details of the measurements, the reader is referred to the papers by Pascioni et al. ${ }^{19}$ and Murayama et al. ${ }^{20}$

Closed-wall wind tunnel tests in the Florida State Aeroacoustic Tunnel (FSAT) with a $0.914 \mathrm{~m} \times 1.219 \mathrm{~m} \times 3.048 \mathrm{~m}$ (Height $\times$ Width $\times$ Length) test section used a combination of surface and offbody measurements to characterize the slat cove flow field. Measured mean surface pressures compared well with numerical predictions for the free-air configuration obtained using time-accurate simulations. Frequency spectra and spanwise coherence of unsteady surface pressures on the slat surface were measured and the characteristics of narrowband peaks in the spectra were investigated at various flow speeds and angles of attack. SPIV measurements provided global information about the shear layer characteristics and turbulence statistics to enable comparison with the numerical simulations.

Synergistic measurements of the 30P30N configuration from BANC-III Workshop were obtained in a $2 \mathrm{~m} \times 2 \mathrm{~m}$ hard-wall wind tunnel at the Japan Aerospace Exploration Agency (JAXA). Performed as part of a collaborative effort on airframe noise between JAXA and NASA, the model geometry and majority of instrumentation details are identical to the model tested at FSU with the exception of a larger span. For an angle of attack up to 10 degrees, the mean surface $\mathrm{Cp}$ distributions agreed well with free-air steady RANS predictions at a corrected angle of attack. After employing suitable acoustic treatment for the brackets and end-wall effects, an approximately 2-D noise source map was obtained from microphone array measurements focused on a selected volume encompassing the slat cove and the gap region between the slat and the main wing leading edge. The nearly 2-D map provides an encouraging prognosis for generating a measurement database that can eventually be used for comparison with free-air numerical simulations.

This first phase of FSU and JAXA measurements met the objective of providing nearfield data for a high lift flow field with good two-dimensionality (including static and dynamic pressure at select locations and spanwise coherence along the slat and the main wing) and qualitative acoustics maps based on a phased array of microphones within a closed hard-wall test section. Future wind tunnel entries will target additional measurements of unsteady surface pressure including spanwise coherence via unsteady pressure sensitive paint (PSP), along with acoustic measurements in an anechoic, Kevlar-wall test section. Only a limited subset of the measurements in the FSU and JAXA facilities will be used in section IV to meet the objectives of the baseline assessment described in this paper. For additional findings from these two tests, the reader is referred to Pascioni et al. ${ }^{19}$ and Murayama et al. ${ }^{20}$ respectively.

Category 7 at the BANC-III Workshop included five different government laboratories, two commercial CFD code developers, two industry organizations, and academic institutions from seven countries. Observations concerning the computational datasets are presented next. With the exception of one submission (identified as Submission 09 below) for an incompressible flow, all remaining simulations were performed for compressible flows. The range of turbulence treatment included none (laminar), DDES, IDDES, NLDE, and VLES. Although almost all of the submissions used viscous, no-slip conditions on solid surfaces, contribution 02 actually employed a slip boundary condition (simple wall shear stress model based on skin friction). Despite the modest Reynolds number involved, all simulations were run in fullyturbulent mode (or fully-laminar in Submission 10 that did not employ any turbulence model), i.e., no transition model was used. No tripping was used in the data considered herein, and the slat boundary layer approaching the cusp is expected to have been quasi-laminar even in the computations employing a turbulence model. Limited measurements involving boundary layer trips in the JAXA facility (which may or may not have successfully tripped the low Reynolds number, favorable pressure gradient boundary layer ahead of the cusp) revealed a noticeable increase in spectral levels associated with narrowband peaks in the nearfield and farfield spectra. ${ }^{20}$

Category 7 contributions during the BANC-III Workshop also provided a unique opportunity to assess the human (i.e., user) element of the computational process. Contributions 15 and 19 involved the same commercial flow and acoustic solvers (albeit with slightly different versions because of the difference in timeframes), allowing a limited assessment of the sensitivity to user characteristics, reflected mainly through the choice of domain and gridding strategy (which also tends to be dictated by available computational resources). Unfortunately, simulation 19 was based on previously performed computations and, hence, could only provide a limited subset of solution metrics targeted for the BANC-III comparisons. 
One of the simulations (Submission 08) was not performed for the conditions of the BANC-III workshop. It was performed as part of an independent effort and involved a slightly lower angle of attack (4 deg) and used the $30 \mathrm{P} 30 \mathrm{~N}$ geometry prior to the modification of the slat contour. However, these changes were deemed sufficiently minor for the baseline assessment targeted in this workshop, so that their data has been included in the overall comparison presented in this paper. However, the reader should keep the underlying differences in mind while interpreting any comparisons involving Submission 08 predictions.

Specific metrics for the computational processes associated with the contributed solutions are listed in Tables 2 through 5. Table 2 outlines the grid characteristics (i.e., computational domain, grid resolution, and discretization technique) based on information provided by the participants. Blank cells denote information that was missing in a number of submissionss. Temporal parameters of the simulations are listed in Table 3, whereas Table 4 outlines the required computational resources. Even though the turnaround times associated with the grid generation and postprocessing phases are also important aspects of the computational resources, this information was not available in a majority of the submissionss and, hence, has not been included. Parameters associated with the propagation phase of the airframe noise prediction process are listed in Table 5.

Typically, grid metrics for computational predictions of time-averaged high lift flow fields ${ }^{24,25}$ focus on the domain size and various measures of grid resolution such as $y+$ in the region of attached boundary layer flow. For the slat noise problem, grid resolution within the regions of separated flow also becomes important for accurate predictions of noise producing flow structures. However, due to the scale disparity in the structures from different parts of the flow field and insufficient knowledge of which metrics are most important for the slat cove aeroacoustics, no single grid metric for the separated regions would be sufficiently meaningful. For convenience, a small set of grid metrics is proposed in Table 2 and their values, wherever available, have been listed.

For quasi-2-D simulations with periodic spanwise boundary conditions, the width of the spanwise domain is a free parameter and would likely have a significant impact on the farfield noise characteristics. A minimum spanwise width of $0.0508 \mathrm{~m}$ was suggested for the baseline comparison targeted in the BANC-III Workshop. However, because the value of this parameter has a direct influence on both the quality and the cost of the simulation, participants were allowed to make their own choice. As seen from Table 2, the spanwise width used by the Category 7 participants varied from a minimum value of $0.0151 \mathrm{~m}$ (Submission 08) to a maximum of $0.201 \mathrm{~m}$ (Submission 09). The larger spanwise width in Submission 09 only became possible by significantly decreasing in the spanwise resolution. Without exception, all BANCIII contributors used a fixed width of the spanwise domain, even when multiple simulations were performed. This is understandable, given the cost of each simulation. However, a few studies of the effect of spanwise domain width would be very valuable and could possibly be done as optional, niche contributions.

Although previous 30P30N CFD studies established that a substantial effort would be required for Category 7 simulations, the most efficient solution strategy has not been established, and is likely to be flow solver dependent. Given that a number of participants were starting with little to modest prior experience and that a sufficient knowledge base concerning the optimal grids required for the CFD methods of interest was not available, computational grids for coordinated investigations were not provided ahead of the BANCIII Workshop. The only exceptions were that two sets of block structured grids were made available to interested participants. One set of grids was generated by JAXA on the basis of their extensive experience with prior simulations of this type and involved two different meshes with nominally the same grid distribution, but with factor of 1.5 difference between the respective cell sizes. The medium (i.e., level -1) grid was used by JAXA in their computations (Submission 11) and the fine (level 0) grid was used by the University of Manchester (Submission 12) and by ONERA (Submission 17). This allows a limited assessment concerning the effect of flow solver on the aeroacoustic predictions. A similar comparison of this type was made possible at NASA Langley, where a different level -1 grid was used with two different solvers, allowing additional comparisons between different solvers.

Multiple participants performed simulations on more than a single grid as reflected by Submissions 03, 04, and 05 by NASA Langley, Submissionss 06 and 07 by KHI, and Submissions 13 and 14 by NASA Ames. Additionally, the PowerFLOW computations by NASA and Exa (Submission 15) were performed on three different grids with a factor of 2 increase in grid resolution along every direction from grid level -2 to grid level -1 , and a factor of 1.5 increase from grid level -1 to grid level 0 . PowerFLOW employs local time stepping, with the finest grid level being updated every time step, the second finest every 2 time steps, the third every 4 time steps, and so on. The average number of elements being updated per time step is called 
Fine Equivalent Voxels (F.E. Voxels as noted under $\mathrm{N}_{\mathrm{v}}$ in Table 2). Submissions 03, 04, and 05 also involved a successive doubling of the resolution in every direction.

In prior BANC Workshops, ${ }^{11,12}$ we have encouraged certain participants to contribute specific additional computations depending on both the needs of the respective category and the unique capabilities or strengths of their computational process, e.g., participants were encouraged to conduct facility-scale computations to provide insights into the installation effects and the influence of acoustic scattering associated with nearby solid surfaces in the QFF facility used for acoustic measurements of the tandem cylinder model. Such facility-scale aeroacoustic computations $\mathrm{s}^{40,41,42}$ have been a unique contribution of the BANC series of workshops. They become even more critical in the case of high-lift configurations, especially when the test sections involved are relatively modest in size. Initially, however, reliable simulations of this type for the Category 7 configuration were deemed somewhat impractical and/or premature. However, the problem statement for Category 7 included an open-ended component to encourage several optional exercises related to the characterization of spanwise coherence, effect of spanwise domain width, other AoA, and higher Reynolds numbers. The intent was to increase the breadth of the simulation database to allow better interpretations of the simulations for the baseline configuration.

To help address the challenges in further understanding the physics of slat aeroacoustics through computations and measurements, including aeroacoustic modelers (i.e., the theoretical component) at the outset was deemed important to the success of this category. Modeling enriches the insights gained from the computational and experimental studies, provides extra fodder for the other two groups, and, in turn, benefits from the access to the rich and evolving database of simulations and measurements. Such access would enable an improved assessment of the limitations of specific assumptions and/or simplifications in the theory, hence contributing to the development of improved and better validated physics based models for slat noise. We were somewhat disappointed that only one contribution of this type was received during the BANC-III Workshop (Submission 18).

Similar to Submission 08, the final contribution from Table 1 (Submission 20) involved the original $30 \mathrm{P} 30 \mathrm{~N}$ configuration and a higher Re of $9.1 \times 10^{6}$. This ambitious set of simulations included WMLES solutions at 15 different angles of attack. In spite of not including any unsteady disturbances as part of the freestream environment, these simulations were able to capture boundary layer transition as part of the solution process, and the predicted transition locations agreed surprisingly well with those measured in experiments. The simulation campaign was undertaken separately from the BANC series of workshops and, hence was not designed to address the data submission requirements of Category 7. Despite that constraint, it brought considerable value to this category, similar to Submission 08. In particular, Submission 20 was in a unique position to map the variations in slat cove aeroacoustics across the entire range of AoA and, furthermore, to address the effects of higher Re on the slat cove dynamics in general and the NBPs in particular. Unfortunately, no data was submitted for comparison with the Category 7 simulations at a lower Reynolds number.

Table 1 List of Submissions (continued)

\begin{tabular}{|c|l|l|l|l|l|}
\hline ID & $\begin{array}{l}\text { Facility/Flow } \\
\text { Solver }\end{array}$ & Contributors & Methodology & $\begin{array}{l}\text { Turbulence } \\
\text { Model }\end{array}$ & Extra Information \\
\hline N/A & $\begin{array}{l}\text { Basic } \\
\text { Aerodynamic } \\
\text { Research } \\
\text { Tunnel } \\
\text { (BART) }\end{array}$ & $\begin{array}{l}\text { Jenkins and Neuhart } \\
\text { (NASA Langley) }\end{array}$ & Experiment & N/A & $\begin{array}{l}\text { Solid wall wind tunnel } \\
\text { with planar and SPIV } \\
\text { measurements }\end{array}$ \\
\hline 00 & $\begin{array}{l}\text { FSU Wind } \\
\text { Tunnel }\end{array}$ & $\begin{array}{l}\text { Pascioni and Cattafesta } \\
\text { (Florida State } \\
\text { University), Choudhari } \\
\text { (NASA Langley) }\end{array}$ & Experiment & N/A & $\begin{array}{l}\text { Solid wall wind tunnel } \\
\text { with SPIV and } \\
\text { steady+unsteady } \\
\text { surface pressure } \\
\text { measurements }\end{array}$ \\
\hline 01 & $\begin{array}{l}\text { JAXA LWT2 } \\
\text { Wind Tunnel }\end{array}$ & $\begin{array}{l}\text { Nakakita, Murayama, } \\
\text { Yamamoto, Ura, and Ito } \\
\text { (JAXA), Choudhari } \\
\text { (NASA Langley) }\end{array}$ & Experiment & N/A & $\begin{array}{l}\text { Solid wall wind tunnel } \\
\text { with microphone array } \\
\text { and steady+unsteady } \\
\text { surface pressure } \\
\text { measurements }\end{array}$ \\
\hline
\end{tabular}

10

American Institute of Aeronautics and Astronautics 
Table 1 List of Submissions (concluded)

\begin{tabular}{|c|c|c|c|c|c|}
\hline 02 & FENICS & $\begin{array}{l}\text { Vilela de Abreu, } \\
\text { Hoffman, and Jansson } \\
(\mathrm{KTH})\end{array}$ & $\begin{array}{l}\text { ILES + slip BC } \\
\text { (wall shear } \\
\text { stress model } \\
\text { based on } C_{f} \text { ) }\end{array}$ & N/A & $\begin{array}{l}\text { FEM with adjoint } \\
\text { based adaptive mesh } \\
\text { refinement }\end{array}$ \\
\hline 03 & $\begin{array}{l}\text { CFL3D } \\
\text { (Grid -2) }\end{array}$ & $\begin{array}{l}\text { Lockard } \\
\text { (NASA Langley) }\end{array}$ & $\begin{array}{l}\text { Zonal DDES } \\
\text { (RANS over } \\
\text { flap) }\end{array}$ & SST & $\begin{array}{l}\text { Finite-volume, block } \\
\text { structured }\end{array}$ \\
\hline 04 & $\begin{array}{l}\text { CFL3D } \\
\text { (Grid -1) }\end{array}$ & & Zonal DDES & SST & \\
\hline 05 & $\begin{array}{l}\text { CFL3D } \\
\text { (Grid 0) }\end{array}$ & & Zonal DDES & SST & \\
\hline 06 & $\begin{array}{l}\text { CFLOW } \\
\text { (Grid 0) }\end{array}$ & $\begin{array}{l}\text { Ueno (Kawasaki Heavy } \\
\text { Industries, Japan) }\end{array}$ & DDES & SA & $\begin{array}{l}\text { Unstructured hybrid } \\
\text { grid (polyhedral }+ \\
\text { layered grid) with } \\
\text { Octree AMR }\end{array}$ \\
\hline 07 & $\begin{array}{l}\text { CFLOW } \\
\text { (Grid -1) }\end{array}$ & & DDES & SA & \\
\hline 08 & ElaN3D & $\begin{array}{l}\text { Knacke and Thiele } \\
\text { (Technical Univ. of } \\
\text { Berlin) }\end{array}$ & DDES & $\begin{array}{l}\text { Strain } \\
\text { adaptive } \\
\text { linear SA } \\
(0.09 \% \mathrm{Tu})\end{array}$ & $\begin{array}{l}\text { Block structured, } \\
\text { Pressure-based finite- } \\
\text { volume (4-deg AoA, } \\
\text { old geometry) }\end{array}$ \\
\hline 09 & $\begin{array}{l}\text { STAR-CCM+ } \\
(\mathrm{M}=0)\end{array}$ & Dahan (KTH) & IDDES & SST & $\begin{array}{l}\text { Unstructured grid, } \\
\text { mostly hexahedral } \\
\text { (Preliminary solution) }\end{array}$ \\
\hline 10 & $\begin{array}{l}\text { Unstructured } \\
\text { Cartesian Flow } \\
\text { Solver }\end{array}$ & $\begin{array}{l}\text { Tamaki and Imamura } \\
\text { (Univ. of Tokyo) }\end{array}$ & Laminar & N/A & $\begin{array}{l}\text { Octree Cartesian grid } \\
\text { with immersed } \\
\text { boundary }\end{array}$ \\
\hline 11 & $\begin{array}{l}\text { UPACS-LES } \\
\text { (Grid -1) }\end{array}$ & $\begin{array}{l}\text { Murayama, Yamamoto, } \\
\text { Tanaka, Amemiya, } \\
\text { Hirai, and Imamura } \\
\text { (JAXA) }\end{array}$ & DDES & SA & \\
\hline 12 & $\begin{array}{l}\text { STAR-CCM+ } \\
(\text { Grid 0) }\end{array}$ & $\begin{array}{l}\text { Ashton (U. Manchester), } \\
\text { West (CD-Adapco), } \\
\text { Mendonca }\end{array}$ & IDDES & SST & \\
\hline 13 & $\begin{array}{l}\text { LAVA } \\
\text { (Grid -2) }\end{array}$ & $\begin{array}{l}\text { Housman and Kiris } \\
\text { (NASA Ames) }\end{array}$ & DDES & SA & \\
\hline 14 & LAVA(Grid 0) & & DDES & SA & \\
\hline 15 & $\begin{array}{l}\text { PowerFLOW } \\
\text { (Exa, NASA) }\end{array}$ & $\begin{array}{l}\text { Choudhari and Lockard } \\
\text { (NASA Langley), } \\
\text { Ribeiro, Fares, and } \\
\text { Casalino (Exa) } \\
\end{array}$ & VLES & $\begin{array}{l}\text { Modified } \\
\text { RNG } k-\varepsilon \\
\text { model for } \\
\text { SGS }\end{array}$ & $\begin{array}{l}\text { Lattice Boltzmann } \\
\text { with surface elements } \\
\text { (surfels) }\end{array}$ \\
\hline 16 & $\begin{array}{l}\text { OVERFLOW } \\
\text { (Grid -1) }\end{array}$ & $\begin{array}{l}\text { Lockard } \\
\text { (NASA Langley) }\end{array}$ & Zonal DDES & SST & $\begin{array}{l}\text { Finite-Difference, } \\
\text { overset grids }\end{array}$ \\
\hline 17 & FUNk & Terracol (ONERA) & NLDE & SA & \\
\hline 18 & $\begin{array}{l}\text { DLR Hybrid } \\
\text { CFD-CAA }\end{array}$ & $\begin{array}{l}\text { Ewert and Boenke } \\
\text { (DLR) }\end{array}$ & RANS + CAA & SST & $\begin{array}{l}\text { Discontinuous } \\
\text { Galerkin for CAA }\end{array}$ \\
\hline 19 & $\begin{array}{l}\text { PowerFLOW } \\
\text { (Embraer, } \\
\text { USP) }\end{array}$ & $\begin{array}{l}\text { Simoes and Bonatto } \\
\text { (Embraer) Souza and } \\
\text { Medeiros (Univ. Sao } \\
\text { Paolo) }\end{array}$ & VLES & $\begin{array}{l}\text { Modified } \\
\text { RNG } k-\varepsilon \\
\text { model for } \\
\text { SGS }\end{array}$ & \\
\hline 20 & CharLES & $\begin{array}{l}\text { Bodart (DAEP/ISAE, } \\
\text { Toulouse), Larsson } \\
\text { (Univ. of Maryland), } \\
\text { Moin (Stanford Univ.) }\end{array}$ & WMLES & & $\begin{array}{l}\text { High-Re LES }(\mathrm{Re}= \\
\left.9.1 \times 10^{6}\right) \text { with focus on } \\
\mathrm{C}_{\mathrm{L}}{ }^{\max }\end{array}$ \\
\hline
\end{tabular}


Table 2 Grid Characteristics

\begin{tabular}{|c|c|c|c|c|c|c|c|c|c|c|c|c|}
\hline ID & Spatial discretization & $\mathrm{O}_{\mathrm{c}}$ & $\begin{array}{c}\mathrm{N}_{\mathrm{V}} \\
\text { (million) }\end{array}$ & \begin{tabular}{|c|}
$\mathrm{N}_{\mathrm{C}}$ \\
(million)
\end{tabular} & $L_{x y} / c$ & $\begin{array}{c}L_{z} \\
(m)\end{array}$ & $\begin{array}{l}\Delta_{\mathrm{SL}, \mathrm{C}} \\
(\mathrm{mm})\end{array}$ & $\begin{array}{r}\Delta_{\mathrm{SL}, \mathrm{R}} \\
(\mathrm{mm}) \\
\end{array}$ & \begin{tabular}{|c|}
$\begin{array}{c}\Delta_{\mathrm{z}} \\
(\mathrm{mm})\end{array}$ \\
\end{tabular} & $\begin{array}{l}\Delta_{\mathrm{BL}, \mathrm{S}} \\
(\mathrm{mm}) \\
\end{array}$ & $y+$ & $\mathrm{n}_{\mathrm{TE}, \mathrm{S}}$ \\
\hline 0 & & & & & & 0.914 & & & & & & \\
\hline 1 & & & & & & 2.0 & & & & & & \\
\hline 2 & & 2 & 1.3 & $\begin{array}{l}6.6 \\
\text { (after } \\
7 \\
\text { refine } \\
\text { ments) }\end{array}$ & 23 & 0.26 & N/A & & & & N/A & \\
\hline 3 & $\begin{array}{l}\text { Cell-centered finite- } \\
\text { volume, MUSCL, } \\
\text { Roe }\end{array}$ & 3 & & 9.9 & 25 & 0.0508 & $4 \mathrm{e}-6$ & & & $3 e-3$ & & \\
\hline 4 & & & & 73.2 & 25 & 0.0508 & & & & & & \\
\hline 5 & & & & 601.0 & 25 & 0.0508 & & & & & & \\
\hline 6 & \begin{tabular}{|l|} 
Cell-centered finite- \\
volume, MUSCL, \\
simple low dissipation \\
AUSM
\end{tabular} & 2 & & 38.6 & & 0.1016 & & & & 0.05 & 0.5 & 16 \\
\hline 7 & & & & 13.9 & & 0.1016 & & & & 0.05 & 0.5 & $7 / 2$ \\
\hline & $\begin{array}{l}\text { cell-centered finite- } \\
\text { volume, TVD-central } \\
\text { hybrid with } \\
\text { generalized Rhie- } \\
\text { Chow interpolation, } \\
\text { hanging node block } \\
\text { interfaces } \\
\end{array}$ & & & 25.0 & 10 & 0.0151 & & $\begin{array}{l}0.38 \\
\text { (slat } \\
\text { cove) }\end{array}$ & 0.38 & & $<1$ & \\
\hline & $\begin{array}{l}\text { upwind-bounded } \\
\text { central hybrid }\end{array}$ & 2 & & 9.4 & 11 & $\begin{array}{l}0.201 \\
\text { free- } \\
\text { slip }\end{array}$ & & 1.0 & & & 25 & \\
\hline 10 & upwind biased SLAU & 4 & & 9.4 & 200 & 0.0418 & 0.17 & 0.17 & 0.68 & 0.087 & 10 & 8 \\
\hline 11 & $\begin{array}{l}\text { low dissipation } \\
\text { upwind (SLAU) }\end{array}$ & 3 & & 22.4 & 100 & 0.0508 & & 0.28 & 0.28 & & 0.81 & \\
\hline 12 & upwind-central hybrid & 2 & & 73.0 & 100 & 0.0508 & & 0.19 & & & 0.54 & \\
\hline 13 & $\begin{array}{l}\text { central with } 5^{\text {th }} \text {-order } \\
\text { WENO based matrix } \\
\text { dissipation (optimal } \\
\text { weights) }\end{array}$ & 4 & & 56.5 & & 0.0508 & & & $0.5-1.9$ & & & \\
\hline 14 & & & & 189.9 & & 0.0508 & & & \begin{tabular}{|l|}
$0.25-$ \\
1.9
\end{tabular} & & & \\
\hline 15 & LBM & 2 & \begin{tabular}{|l|}
218 \\
(F.E. \\
Voxels
\end{tabular} & 1026.0 & 100 & 0.05715 & & 0.037 & & & & \\
\hline 16 & & 5 & & 73.2 & & 0.0508 & & & & & & \\
\hline 17 & $\begin{array}{l}\text { finite-volume, } \\
\text { modified AUSM+P }\end{array}$ & 2 & & 73.0 & 100 & 0.06858 & & & & & & \\
\hline 18 & \begin{tabular}{|l} 
DG with 3 \\
DOFs/edge, 10 \\
DOFs/triangle for \\
CAA simulation \\
\end{tabular} & 4 & 0.2 & 0.4 & & $\begin{array}{l}0.0 \\
(2 \mathrm{D})\end{array}$ & & \begin{tabular}{|l|}
3 ppw \\
up to 10 \\
$\mathrm{kHz}$
\end{tabular} & & & & \\
\hline 19 & LBM & 2 & \begin{tabular}{|l|}
73 \\
(F.E. \\
Voxels
\end{tabular} & 73 & $\approx 10$ & 0.0508 & & & & & \begin{tabular}{|l|}
25 \\
(me- \\
an)
\end{tabular} & \\
\hline 20 & & & 70 & & & $0.016 c$ & & & & & & \\
\hline
\end{tabular}

12

American Institute of Aeronautics and Astronautics 
Table 3 Temporal Characteristics of Simulations

\begin{tabular}{|c|c|c|c|c|c|c|c|c|c|c|}
\hline ID & $\begin{array}{l}\text { Time } \\
\text { advanceme } \\
\text { nt }\end{array}$ & $\begin{array}{l}\Delta \mathrm{t} \\
(\mathrm{sec})\end{array}$ & $\mathrm{N}_{\text {sub }}$ & $\mathrm{T}_{\text {sampling }}$ & \begin{tabular}{|c|}
$f_{\text {sampling }}$ \\
$(\mathrm{kHz})$
\end{tabular} & $\mathrm{N}_{\mathrm{t}, \text { transient }}$ & $\mathrm{N}_{\mathrm{t}, \text { sampling }}$ & $\begin{array}{l}\text { Sampling } \\
\text { interval in } \\
\text { flow- } \\
\text { through } \\
\text { times } \\
\left(\mathrm{T}_{\text {sampling }}\right. \\
c / U) \\
\end{array}$ & \begin{tabular}{|l|} 
No of \\
averages \\
for \\
acoustic \\
spectrum
\end{tabular} & $\begin{array}{l}\text { Bin } \\
\text { width } \\
(\mathrm{Hz})\end{array}$ \\
\hline 0 & & & & & & & & & & \\
\hline 1 & & & & & & & & & & \\
\hline 2 & & & & & & & & & & \\
\hline 3 & $\begin{array}{l}\text { Implicit } \\
2^{\text {nd }} \text {-order }\end{array}$ & & & & & & & & & \\
\hline 4 & & & & & & & $50 \mathrm{~K}$ & & & \\
\hline 5 & & & & & & & $33 \mathrm{~K}$ & & & \\
\hline 6 & $\begin{array}{l}2^{\text {nd }} \text {-order } \\
\text { matrix free } \\
\text { Gauss- } \\
\text { Seidel } \\
\text { implicit }\end{array}$ & $1.18 \mathrm{e}-6$ & 16 & $11.8 \mathrm{e}-6$ & 85 & $100 \mathrm{~K}$ & $\begin{array}{l}68 \mathrm{~K} \\
(0.08 \mathrm{~s})\end{array}$ & 10.11 & 12 & $83 \mathrm{~Hz}$ \\
\hline 7 & & & 12 & & & $100 \mathrm{~K}$ & $\begin{array}{l}50 \mathrm{~K} \\
(0.06 \mathrm{~s})\end{array}$ & 7.44 & 8 & \\
\hline 8 & $\begin{array}{l}2^{\text {nd }} \text {-order } \\
\text { implicit }\end{array}$ & $\begin{array}{l}1 . e-6 \\
(25 \text { pts } \\
\text { per } \\
\text { period } \\
\text { for } 40 \\
\mathrm{kHz})\end{array}$ & & & & $40 \mathrm{~K}$ & $60 \mathrm{~K}$ & 7.35 & & \\
\hline 9 & \begin{tabular}{|l|}
$2^{\text {nd }}$-order \\
implicit
\end{tabular} & $5 e-6$ & & $2.5 \mathrm{e}-5 \mathrm{~s}$ & & $0.015 \mathrm{~s}$ & $0.06 \mathrm{~s}$ & 7.7 & \begin{tabular}{|l|l|} 
None in \\
Span
\end{tabular} & \\
\hline 10 & $\begin{array}{c}2^{\text {nd }}-\text { order } \\
\text { implicit }\end{array}$ & $1.3 e-6$ & 8 & $5.2 \mathrm{e}-3 \mathrm{~s}$ & 192 & $0.069 \mathrm{~s}$ & $0.0315 \mathrm{~s}$ & 12.8 & 11 & 151 \\
\hline 11 & \begin{tabular}{|l|}
$2^{\text {nd }}$-order \\
Euler \\
implicit \\
\end{tabular} & $0.22 \mathrm{e}-6$ & 5 & & 4569 & $0.18 \mathrm{~s}$ & $\begin{array}{ll}360 & \mathrm{~K} \\
(0.079 \mathrm{~s})\end{array}$ & 10.1 & 21 & $\begin{array}{c}35 \\
\text { (56 for } \\
\text { acoustic) }\end{array}$ \\
\hline 12 & & & & & & $\begin{array}{l}0.23 \\
\text { (nearfield) }\end{array}$ & & & 6 & 26 \\
\hline 13 & $\begin{array}{l}2^{\text {nd }} \text {-order } \\
\text { implicit, } \\
\text { alternating } \\
\text { line Jacobi }\end{array}$ & $1 e-6$ & $11-16$ & & & & & & $5-7$ & \\
\hline 14 & & & & & & & & & & \\
\hline 15 & Explicit & 6.1e-6 & & & 682 & $\begin{array}{l}4 \mathrm{M} \\
>0.23 \mathrm{~s} \\
\end{array}$ & $\begin{array}{l}4.9 \mathrm{M} \\
0.3 \mathrm{~s} \\
\end{array}$ & 40 & 8 & 13 \\
\hline 16 & & & & & & & & & & \\
\hline 17 & $\begin{array}{l}2^{\text {nd }} \text {-order } \\
\text { backward } \\
\text { Euler with } \\
\text { Newton }\end{array}$ & $0.2 \mathrm{e}-6$ & $\begin{array}{l}\text { Dyna } \\
\text { mic } \\
\text { subiter } \\
\text { ations }\end{array}$ & & 500 & $\begin{array}{l}\text { 50K }(0.01 \\
\text { s) }\end{array}$ & $\begin{array}{l}580 \mathrm{~K}, \\
0.116 \mathrm{~s}\end{array}$ & 14.8 & 11 & 50 \\
\hline 18 & & & & & & $200 \mathrm{~K}$ total & & & & \\
\hline 19 & Explicit & $3.3 \mathrm{e}-7$ & & & & & & & & 50 \\
\hline 20 & Explicit & & & & & & & & & \\
\hline
\end{tabular}


Table 4 Computational Resources

\begin{tabular}{|c|c|c|c|c|c|}
\hline ID & CPU Hardware & Interconnect & $\begin{array}{l}\text { Number } \\
\text { of cores }\end{array}$ & $\begin{array}{l}\text { Wall clock time } \\
\text { per time step } \\
\text { (seconds) }\end{array}$ & $\begin{array}{l}\text { Wall clock time for } \\
1 \text { sec simulation } \\
\text { with } 1,000 \text { cores } * \\
\text { (months) }\end{array}$ \\
\hline 0 & N/A & N/A & N/A & N/A & N/A \\
\hline 1 & N/A & N/A & N/A & N/A & N/A \\
\hline 2 & & & & & \\
\hline 3 & & & & & \\
\hline 4 & $\begin{array}{c}\text { SGI Altix ICE } \\
\text { Xeon(R) X5675 (Westmere), } \\
3.07 \mathrm{GHz}\end{array}$ & $\begin{array}{c}\text { Infiniband } \mathbb{R} \\
\text { QDR }\end{array}$ & 228 & 86.9 & 3.5 \\
\hline 5 & & & 1008 & 226 & 40.6 \\
\hline 6 & Xeon E5-2670 (8 cores) & & 256 & & 1.5 \\
\hline 7 & & & 64 & & 0.3 \\
\hline 8 & SGI Altix 4700 & & 240 & $16 \mathrm{~s}$ & 1.5 \\
\hline 9 & & & & & \\
\hline 10 & HITACHI SR16000 & & 32 & 23 & \\
\hline 11 & & & & & \\
\hline 12 & & & & & \\
\hline 13 & & & & & \\
\hline 14 & & & & & \\
\hline 15 & $\begin{array}{c}\text { SGI Altix ICE } \\
\text { Xeon(R) X5675 (Westmere), } \\
3.07 \mathrm{GHz}\end{array}$ & $\begin{array}{l}\text { Infiniband } \mathbb{R} \\
\text { QDR }\end{array}$ & 3840 & & 1.9 \\
\hline 16 & $\begin{array}{c}\text { SGI Altix ICE } \\
\text { Xeon(R) X5675 (Westmere), } \\
3.07 \mathrm{GHz}\end{array}$ & $\begin{array}{c}\text { Infiniband } \mathbb{} \\
\text { QDR }\end{array}$ & & & \\
\hline 17 & $\begin{array}{l}\text { Nehalem nodes wth } 2 \text { quad-core } \\
\text { Intel Xeon X5560 }\end{array}$ & Infiniband $®$ & 128 & $2 \mathrm{~s}$ & 0.4 \\
\hline 18 & & & 1 & $0.36 \mathrm{~s}$ & 0.002 \\
\hline 19 & Intel Xeon E5420, $2.5 \mathrm{GHz}$ & & 160 & & 0.23 \\
\hline 20 & Sequoia supercomputer & & & & \\
\hline
\end{tabular}

* Perfect scaling assumed from the number of cores used in actual calculation to the reference number of cores (i.e., 1,000) 
Table 5 Acoustic Prediction

\begin{tabular}{|c|c|c|c|c|c|c|}
\hline ID & $\begin{array}{l}\text { Acoustic } \\
\text { Prediction Code }\end{array}$ & $\begin{array}{l}\text { Acoustic Prediction } \\
\text { Methodology }\end{array}$ & \begin{tabular}{|l} 
Spatial-Order \\
and \\
Structured \\
Grid (S)/ \\
Unstructured \\
Grid (U)
\end{tabular} & $\begin{array}{l}\text { Temporal } \\
\text { order }\end{array}$ & $\begin{array}{l}\text { Acoustic } \\
\text { Formulation }\end{array}$ & $\begin{array}{l}\text { Mean flow } \\
\text { convective } \\
\text { effects } \\
\text { included? }\end{array}$ \\
\hline 0 & N/A & & & & & \\
\hline 1 & Microphone Array & & & & & \\
\hline 2 & N/A & N/A & N/A & N/A & N/A & N/A \\
\hline 3 & FWH3D & $\begin{array}{l}\text { FWH Frequency } \\
\text { Domain }\end{array}$ & $2 \mathrm{~S}$ & & & Yes \\
\hline 4 & FWH3D & & & & & \\
\hline 5 & FWH3D & & & & & \\
\hline 6 & CFLOW & $0.05 \mathrm{~mm}$ & & & & $16 / 8$ \\
\hline 7 & CFLOW & $0.05 \mathrm{~mm}$ & & & & \\
\hline 8 & ELAN-3D & $\begin{array}{l}\text { FWH Time-Domain } \\
\text { (source-time } \\
\text { dominant algorithm) }\end{array}$ & $2 \mathrm{~S}$ & 2 & $\begin{array}{l}\text { Farassat } \\
\text { Formulation } \\
\text { 1A }\end{array}$ & \\
\hline 9 & N/A & N/A & N/A & N/A & N/A & N/A \\
\hline 10 & N/A & N/A & N/A & N/A & N/A & N/A \\
\hline 11 & $\begin{array}{l}\text { UPACS-Acoustics } \\
\text { (Grid -1) }\end{array}$ & & & & & \\
\hline 12 & $\begin{array}{l}\text { STAR-CCM+ } \\
\text { (Grid 0) }\end{array}$ & & & & & \\
\hline 13 & LAVA (Grid -2) & & & & & \\
\hline 14 & LAVA (Grid 0) & & & & & \\
\hline 15 & PowerACOUSTICS & FWH & $1 \mathrm{U}$ & 4 & $\begin{array}{l}\text { Forward time } \\
\text { formulation } \\
\text { based on } \\
\text { retarted time } \\
\text { formulation } \\
\text { 1A by Farassat }\end{array}$ & Yes \\
\hline 16 & FWH3D & $\begin{array}{l}\text { FWH Frequency } \\
\text { Domain }\end{array}$ & $2 S$ & & & Yes \\
\hline 17 & N/A & N/A & N/A & N/A & N/A & N/A \\
\hline 18 & & $\begin{array}{l}\text { Acoustic } \\
\text { Perturbation } \\
\text { Equations + FRPM }\end{array}$ & $4 \mathrm{U}$ & & DG & \\
\hline 19 & PowerACOUSTICS & FWH & & & $\begin{array}{l}\text { Forward time } \\
\text { formulation } \\
\text { based on } \\
\text { retarted time } \\
\text { formulation } \\
\text { 1A by Farassat } \\
\end{array}$ & Yes \\
\hline 20 & N/A & N/A & N/A & N/A & N/A & N/A \\
\hline
\end{tabular}




\section{Results}

This section is devoted to a summary of the comparisons involving several aspects of the contributed data for the unsteady nearfield and the acoustic farfield, ranging from flow visualizations, line plots involving profiles of statistical measures and frequency spectra, and different scalar metrics based on either integral or local quantities that allow a quantitative comparison including statistical analysis. The reader is cautioned that some of the conclusions may be influenced by potential errors in data submission, data processing, and/or interpretation. A lot of data was requested from the participants in Category 7, and even more could have been requested but was deferred to a follow-on phase. The sheer breadth of this data increases the probability of occasional errors. A few such errors have already been caught during the analysis presented here. The participants were very cooperative in addressing multiple requests for corrections and for additional information, which made the task of data comparison more manageable and also improved the quality of the findings. As further information in terms of currently missing data and/or corrections to previously submitted data becomes available in the future, this summary report will be updated and made available online through the AIAA website for the BANC-III Workshop. ${ }^{1}$

\section{A. Instantaneous Flow Field}

We begin the data analysis with snapshots of instantaneous spanwise vorticity distributions from the computational solutions and comparisons with PIV based flow images from the FSU facility (Fig. 3). This particular flow visualization provides an instant visual check on the anticipated quality of each simulation. The visual comparison involves various features such as: the trajectory of the shear layer separating from the slat cusp, the range of vorticity scales and the variation in scales along the trajectory, the presence (and relative significance) of vorticity structures with negative spanwise vorticity (which is associated with flow structures generated via eruptions from the viscous flow adjacent to the slat suction surface), shedding of vorticity from the finite thickness trailing edge of the slat, the interaction between vorticity structures convected along the shear layer trajectory and those shed from the trailing edge, and finally, a possible interaction between the unsteady flow structures generated within the slat region and the main-wing leading edge. Despite the broad range of spanwise domain widths and cell sizes reflected in the BANC-III contributions, all of these 3-D solutions indicate a relatively well defined shear layer trajectory bounding the cove region, along with vorticity blobs that compare favorably to not too unfavorably with the structures observed in the PIV images and those expected on the basis of steady RANS solutions. ${ }^{43}$ This favorable comparison underlines the clear superiority of 3-D simulations over those restricted to a 2-D section. ${ }^{30}$ On the visual yardstick at least, the approximate reattachment location of the slat shear layer is nominally similar in most cases. This is an important metric because prior computational studies have pointed out multiple factors in the context of Fig. 1 (such as hot spots in the TKE distribution, proximity to slat trailing edge and gap regions, strong distortion of flow structures due to mean flow acceleration, etc.) that indicate that the dynamics in the vicinity of the reattachment and slat trailing edge/gap regions are particularly relevant to the generation of sound in the slat cove region. On the other hand, a few of the simulations (especially Submissions 02 and 09) include only larger scale spanwise vorticity structures with a delayed shear layer roll-up and they also lack the finer scales induced during shear layer transition. This does not make them any less useful to the collective effort. Given the considerable cost of the time-accurate simulations, determining the benefit of relatively coarse simulations in a practical context id worthwhile. In the case of BANC-III contributions, the simulations with coarser grids represent preliminary efforts by academic groups to develop novel flow solution techniques (adaptive technique for Submission 02, and a high-order Cartesian grid method for Submission 10) or to "kick start the CFD process" during a new foray into airframe noise research. Thus, the fact that the underlying grids are relatively coarse was well understood by those submitting the results. Yet, they chose to make their findings available for the collective comparison to help enable a broader baseline assessment in the true spirit of this workshop; and we firmly believe that the inclusion of their solutions in this summary will provide useful lessons for others in the airframe noise community. 


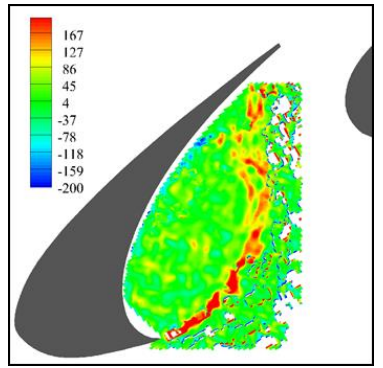

(a) 00

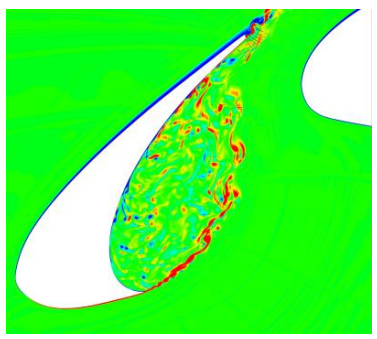

(e) 05

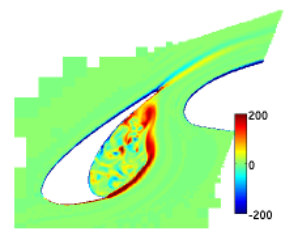

(i) 09

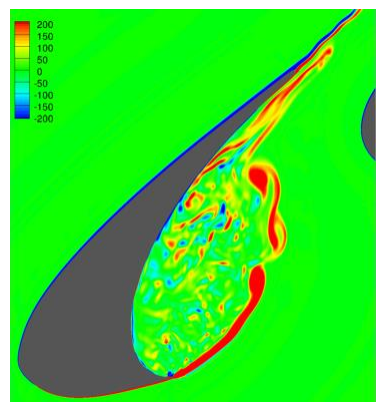

(m) 13

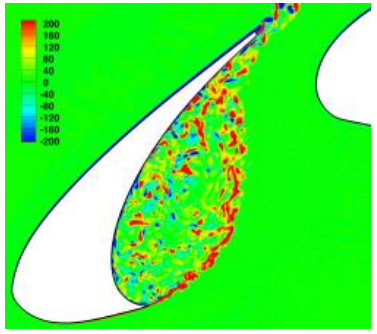

(q) 17

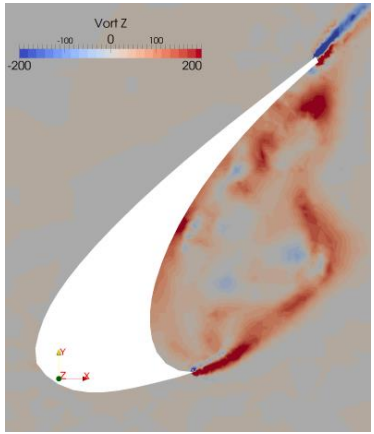

(b) 02

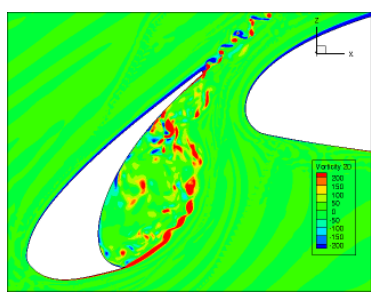

(f) 06

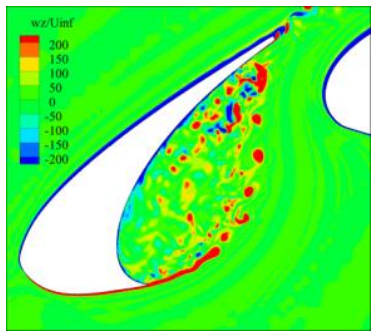

(j) 10

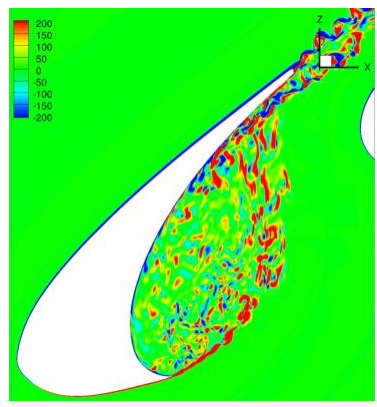

(n) 14

(o) 15

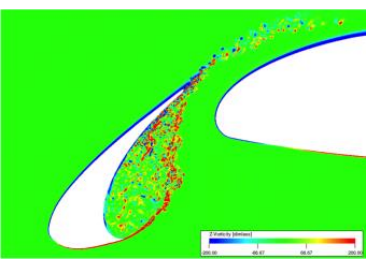

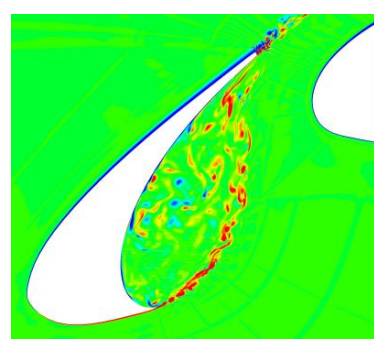

(d) 04

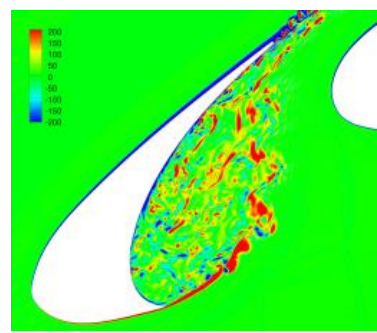

(h) 08

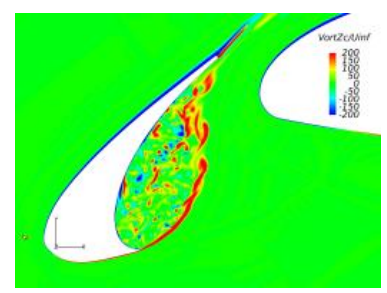

(k) 11

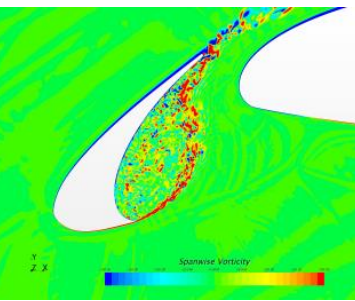

(I) 12

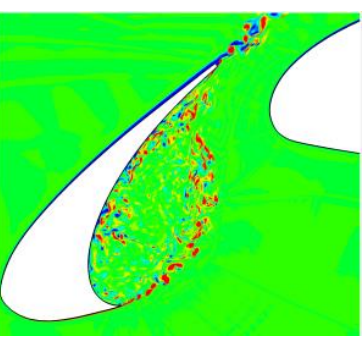

(p) 16

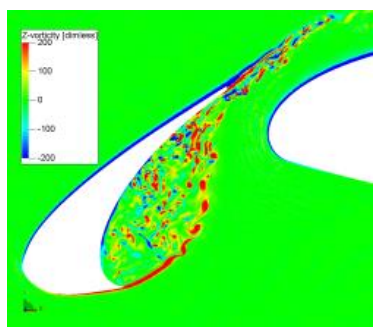

(r) 19

Figure 3. Visualization on instantaneous spanwise vorticity in $x y$ plane. 
As noted in the previous paragraph, the flow structures in the slat cove region are intrinsically 3-D and hence, a planar cross-section of the instantaneous flow field provides only limited insight into the overall dynamics of the flow. To complement the spanwise vorticity contours in Fig. 3, the Category 7 participants were also asked to provide a perspective view of an isosurface of instantaneous streamwise vorticity colored by density (Fig. 4). These images illustrate the evolution of vorticity structures in the shear layer from quasi-2-D Kelvin-Helmholtz rollers near the cusp to increasingly 3-D behavior farther downstream along the shear layer trajectory. The emergence of streamwise elongated flow structures due to vorticity stretching near the gap between the slat and the main wing may also be noted in many cases. The granularity of the fine scale structures varies quite a bit, e.g., the images for Submissions 12, 14, 15, 17, and perhaps, 08 and 10 as well, indicate substantially finer structures than the remaining submissions. The range of scales depends on both the grid resolution and the underlying CFD algorithm. The relatively broad range of scales in Submission 10, despite the relatively coarse grid, may be related to the absence of any SGS or eddy viscosity, together with a $4^{\text {th }}$-order discretization of the convective operator. 


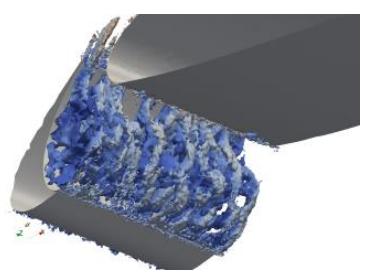

(a) 02

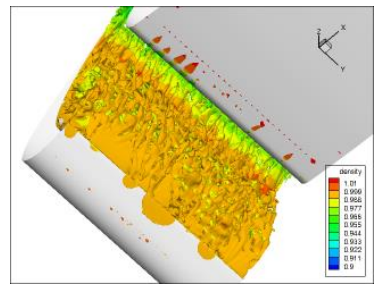

(e) 06

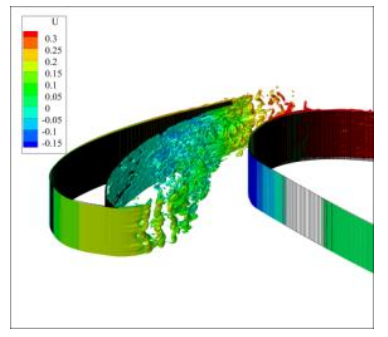

(i) 10

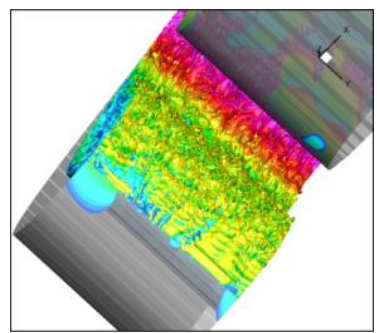

(m) 14

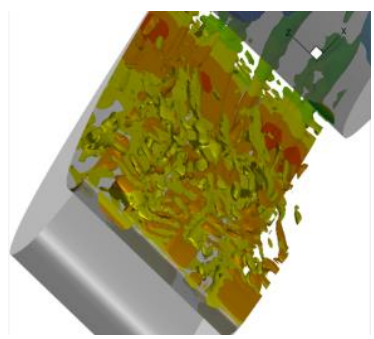

(b) 03

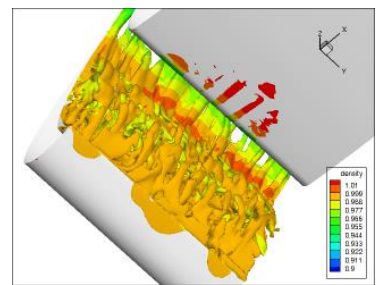

(f) 07

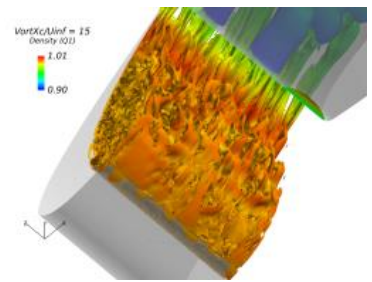

(j) 11

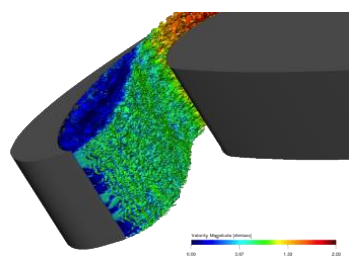

(n) 15

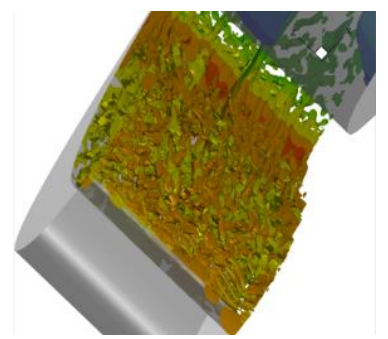

(c) 04

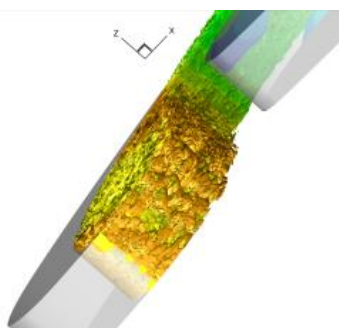

(g) 08

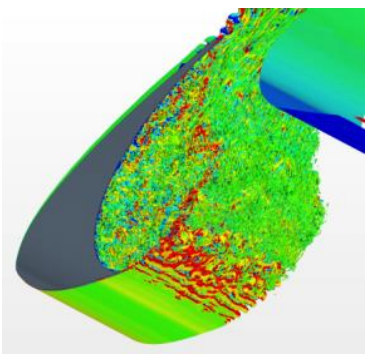

(k) 12

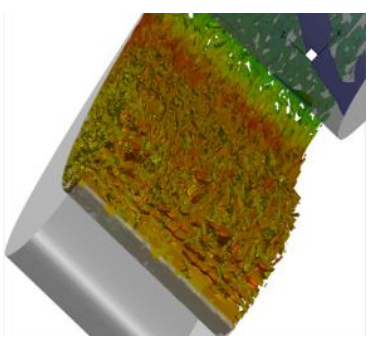

(o) 16

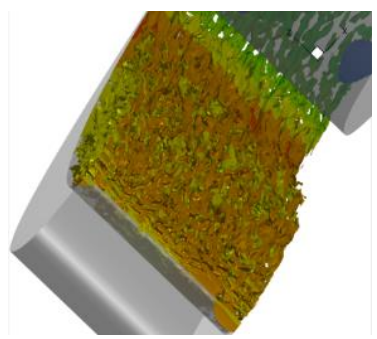

(d) 05

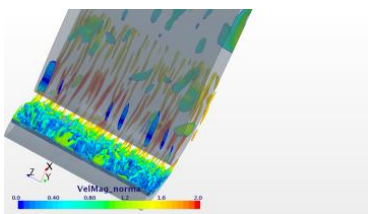

(h) 09

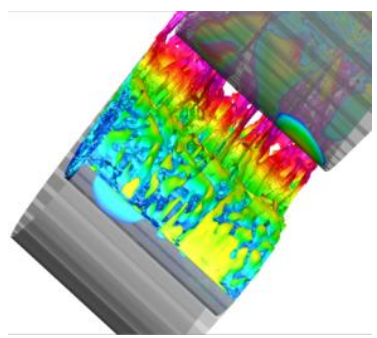

(I) 13

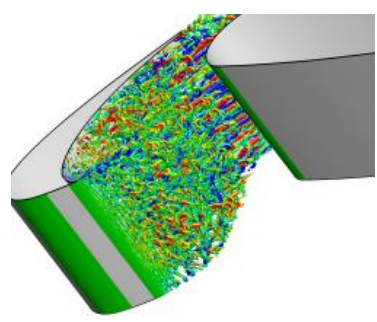

(p) 17

Figure 4. 3-D vorticity structures in slat cove region.

\section{B. Mean and RMS Lift Coefficients}

The mean lift coefficient provides a useful metric to ensure the similarity of underlying flow environment among the various simulations. Furthermore, other workshops such as the CFD Challenge Workshop ${ }^{26}$ and the High Lift Prediction Workshops ${ }^{24,25}$ have focused on the predictions of mean force and moment coefficients obtained primarily from RANS codes. Thus, the $\mathrm{C}_{\mathrm{L}}$ obtained via time accurate simulations is assessed here, albeit for a simpler high-lift configuration and/or within the limited context of the BANC-III Workshop (i.e., a modest Reynolds number and single AoA within the linear portion of $\mathrm{C}_{\mathrm{L}} \mathrm{vs.}$ AoA curve). The distribution of mean lift coefficient, $\mathrm{C}_{\mathrm{L}}$, based on the contributed data is shown in Fig. 5 as a function of the Submission. The green dashed line indicates the median, $\mu$, of the entire set of lift coefficients, and the two dash-dot lines bound the confidence interval $\mu \pm K \sigma$ with $K=3^{1 / 2}$. Here, $\mu$ denotes the sample median (not the arithmetic mean) and $\sigma$ is the standard deviation of the sample. ${ }^{44,45} \mathrm{Hemsch}^{44}$ 
proposed using the various predictions of the same scalar metric as different realizations of the collective computational process, and the scatter among the realizations as a measure of noise (i.e., scatter) in that process. This type of $\mathrm{N}$-version testing enables more meaningful inferences concerning whether the scatter is significant and when the outliers indicate a potentially significant difference in the underlying computational process that may be worth investigating. The choice of $K=3^{1 / 2}$ to estimate the confidence interval (or scatter limits) is based on the assumption of a uniformly distributed PDF, ${ }^{45}$ which appears to be a reasonable choice for the lift coefficient predictions. Similar confidence intervals are shown in subsequent figures where the quantity of interest may be distributed over a positive semi-definite range and the notion of a uniform PDF becomes less tenable. For those cases, the confidence interval shown may be interpreted more loosely, as simply an indicator of variability across the sample.

There are two outliers (Submissions 02 and 09) that fall outside of the confidence interval. These two submissions are already known to be preliminary solutions based on significantly coarser grids than the remaining set, presenting an obvious likely cause for the low $C_{L}$ values in these two submissions and lending partial support for the choice of confidence intervals. The lift coefficient in Submission 03 (which corresponds to the coarsest grid among a set of three computations using the same flow solver but successively refined grid resolution) is also on the lower side, but still well within the confidence interval. As the grid resolution is doubled (Submission 04) and quadrupled (Submission 05), the $C_{L}$ values increase and approach the median value. Interestingly, after discounting the two outliers, the remaining set of predictions cluster in two groups: one group with $C_{L}$ values that are nearly equal or slightly below the median (Submissions 05 to 08, 10, 15, 16, and 18) and the other group with $\mathrm{C}_{\mathrm{L}}$ values that are above the median (Submissions 11, 13, 14, 17, and 19). This difference appears to be correlated with the choice of turbulence model in the hybrid RANS-LES computations, with the SA model yielding higher $\mathrm{C}_{\mathrm{L}}$ values than the SST model. The exceptions to this correlation are Submission 06 and 07 (which also involved the SA model, but provided mean $\mathrm{C}_{\mathrm{L}}$ values that are lower than the remaining computations) and Submission 08 (whose lower $\mathrm{C}_{\mathrm{L}}$ value despite the use of $\mathrm{SA}$ model can be easily explained by the lower angle of attack used in this computation). The $C_{L}$ predictions for Submission 15, which are based on a combination of LBM and VLES with a modified RNG $k-\varepsilon$ model for the SGS, are closer to those based on the SST model. On the other hand, Submission 19, which uses the same VLES/RNG $k-\varepsilon$ model, predicts higher values (closer to the SA group. One significant difference between the two computations (besides gridding strategy and flow solver versions) was the domain size (nearly three times smaller in the case of Submission 19 as seen from the $L_{x y} / c$ values in Table 2), and whether the domain size might account for the observed difference is not clear. Independent 2-D computations (P. Buning, private communication, April 2015) have provided evidence suggesting that the role of turbulence model is a stronger influence on the mean airfoil loading than the size of the computational domain in the $x y$ plane. The 2-D computations also confirm the trend of higher $\mathrm{C}_{\mathrm{L}}$ values with the SA turbulence model than those obtained with the SST model.

If one assumes that the noise contribution from off-body quadrupoles is weak in the low Mach number limit, the noise radiation from the slat is dominated by the surface pressure fluctuations. In that regard, the RMS fluctuations in the overall lift coefficient provides a convenient means of comparing the various computations. In the low frequency limit, most airframe noise problems reduce to dipole noise, and the unsteady lift coefficient corresponds to an integral measure of the strength of the equivalent dipole. Of course, it does not account for the details of the frequency spectrum or the phase relationships between pressure fluctuations along different parts of the surface (which become more significant with increasing frequency). Furthermore, because of the extended nature of the overall high-lift configuration in comparison with the slat chord, the compact source limit is relevant only at very low frequencies (i.e., much lower than the most energetic spectral components of slat noise). Hence, the utility of the RMS lift fluctuation (used by itself) is likely to be highly limited and its inclusion during this first round of detailed comparisons was intended merely as a preliminary exploration of its potential utility. The results in Fig. 6 confirm the suspected limitations of this metric. The amplitudes of the overall lift fluctuation are scattered over a broad range (nearly a factor of five from the minimum to the maximum) and do not show any strong correlation with the either the turbulence model or the RMS lift coefficient for the slat alone. 


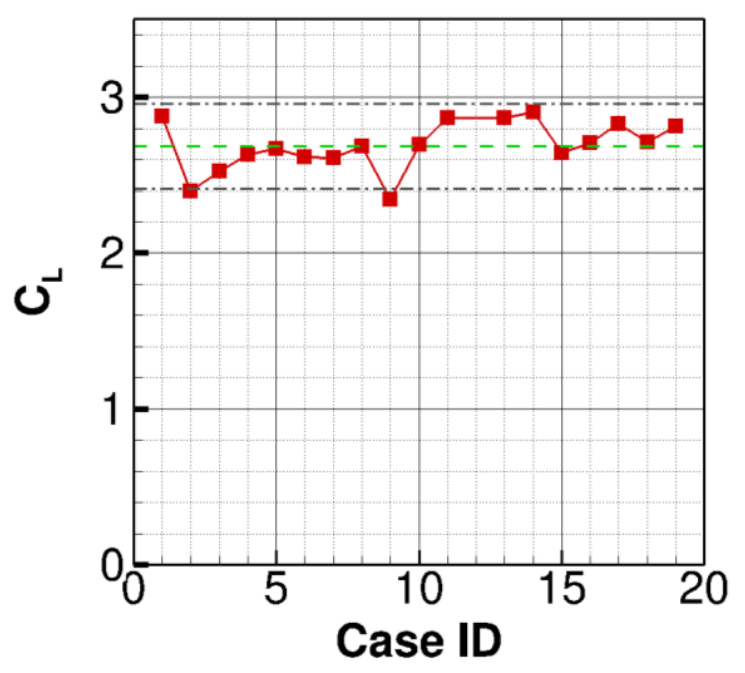

Figure 5. Comparison of mean lift coefficient $C_{L}$.

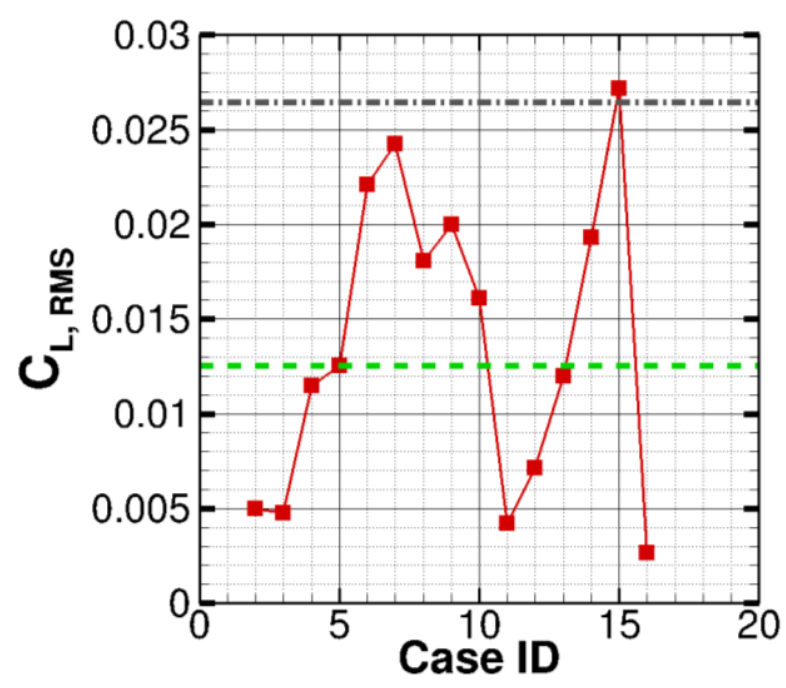

Figure 6. Comparison of RMS fluctuations in $C_{L}$.

The green dashed line indicates the median of the entire set of lift coefficients, and the two dash-dot lines bound a confidence interval.

\section{TKE Distributions}

A collective comparison of the (resolved) $\mathrm{TKE}_{2 \mathrm{D}}$ distribution in the vicinity of the slat is shown in Fig. 7. The 2-D TKE is used because it can also be used to compare with planar PIV measurements from an earlier

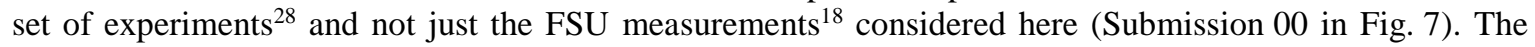
TKE distribution reflects the energy of the hydrodynamic disturbances that are collectively responsible for the noise generation via interaction with the nonuniform mean flow and the airfoil surfaces. The overall noise may be viewed as a convolution of the associated Lighthill stress tensor with a suitable (i.e., tailored) Green's function. The TKE distribution does not include any information about the frequency spectrum and spatial coherences that determine the value of the convolution integral Although the TKE is only a partial measure of the Lighthill stress tensor, it does provide an easily computed metric that is expected to provide some useful information about the accuracy of the flow solution for aeroacoustic prediction. In other words, the TKE distribution is far from being a sufficient (or even dominant) measure for the accuracy of the solution. However, until the precise details of the noise generation process can be pinpointed (which is indeed one of the goals for the BANC Workshops and, hence, the Category 7 as well), we regard the TKE as a relevant metric. If a flow solution were to predict the correct noise spectra while performing very poorly in predicting the TKE features, then we would regard the physical basis of that aeroacoustic prediction to be suspect and/or look for an alternate paradigm for noise generation beyond Fig. 1.

The metrics of interest for the TKE distribution are its qualitative distribution, specific peak regions, and corresponding peak values. As discussed in Ref. 30, the highest levels of TKE occur near the reattachment location as the unsteady structures within the shear layer approach the slat surface and subsequently, either accelerate towards the trailing edge or get convected back towards the slat cusp. The TKE distribution exhibits two local maxima within the reattachment region, one of which lies just ahead of the reattachment location and the other, typically higher maximum occurs along a narrow ridge within the contours. A third (and weaker) maximum is also observed within the slat shear layer and is believed to be associated with the laminar-turbulent transition process. For the most part, the qualitative distribution of TKE appears to be similar in all CFD results, with the most notable differences related to the peak within the first half of the shear layer trajectory. The PIV-based $\mathrm{TKE}_{2 \mathrm{D}}$ distribution does not appear to capture this peak in the shear layer, but we attribute that to the underresolution of the thin shear layer in that region. This peak is also absent from the $\mathrm{TKE}_{2 \mathrm{D}}$ distributions for Submissions 07, 11, 13, and possibly, 06 and 02 . Whether the absence of this peak suggests a possible underresolution in some of these submissions is not clear. More importantly, the influence of this peak on the ability to model the correct physics near the impingement location is not clear, so we merely note that as one of the distinctions between the various solutions. Somewhat surprisingly, the $\mathrm{TKE}_{2 \mathrm{D}}$ distributions for Submissions 07, 9, 11, and 13 appear to lack the peak 
within the near wake region behind the slat trailing edge. The shedding behind the finite thickness trailing edge is a robust feature of the unsteady flow near this slat and even if it does not influence the slat cove noise in the frequency range of interest (less than $10 \mathrm{kHz}$ ), the absence of this peak may indicate inadequate resolution in an otherwise crucial region. The most important takeaway from the $\mathrm{TKE}_{2 \mathrm{D}}$ distributions is that (similar to the spanwise vorticity distributions) all 3-D simulations, regardless of the grid resolution within these simulations, predict a TKE distribution that is qualitatively similar to that inferred from the PIV data.

A comparison of the peak $\mathrm{TKE}_{2 \mathrm{D}}$ levels near the shear layer reattachment location is shown in Fig. 8. For a given flow solver, the peak values increase as the grid is refined and the overall spread among the computational solutions is fairly large, corresponding to a range of $(0.035,0.065)$. Furthermore, there does not appear to be any correlation between the turbulence model and the peak $\mathrm{TKE}_{2 \mathrm{D}}$ levels near the shear layer reattachment location. 


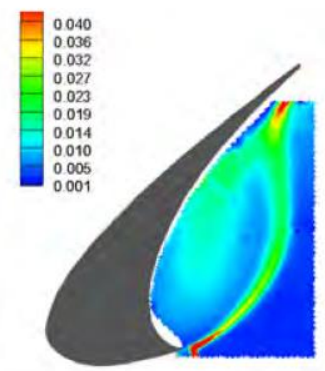

(a) 00

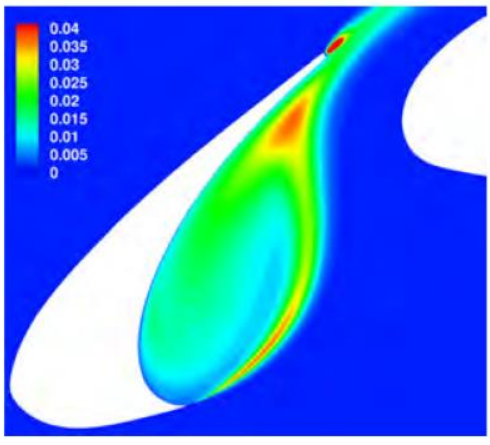

(d) 04

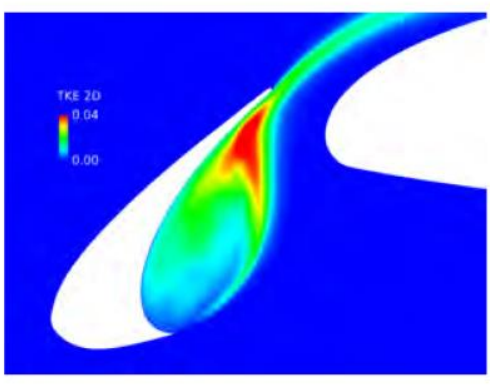

(g) 07

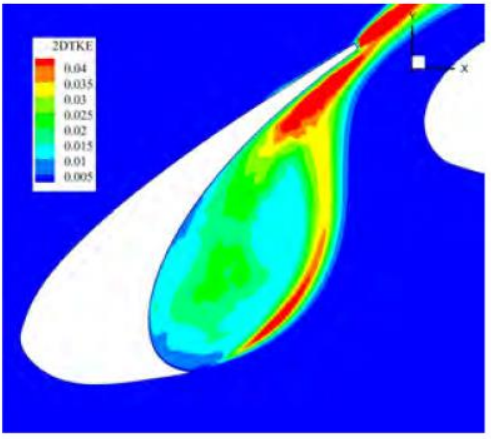

(j) 10

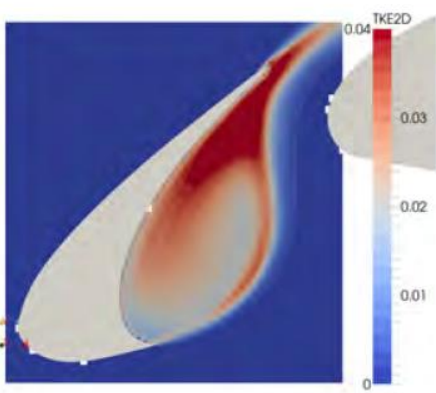

(b) 02

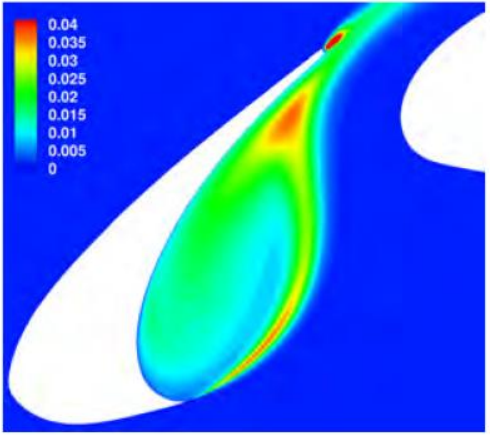

(e) 05

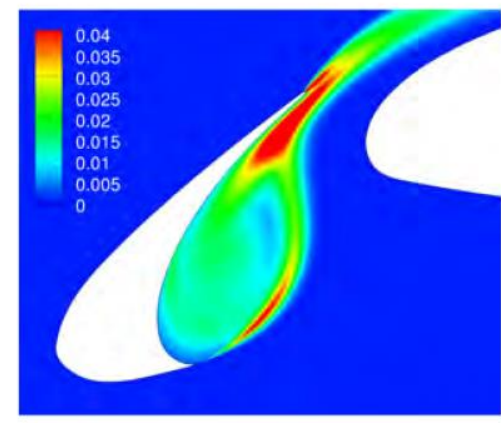

(h) 08

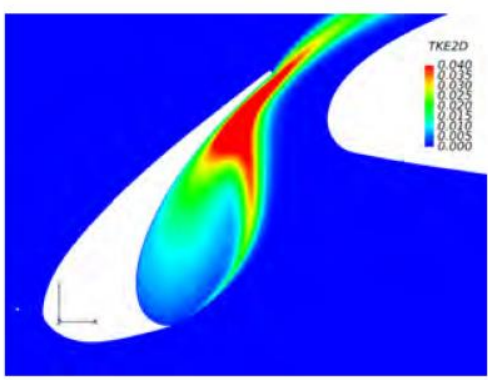

(k) 11

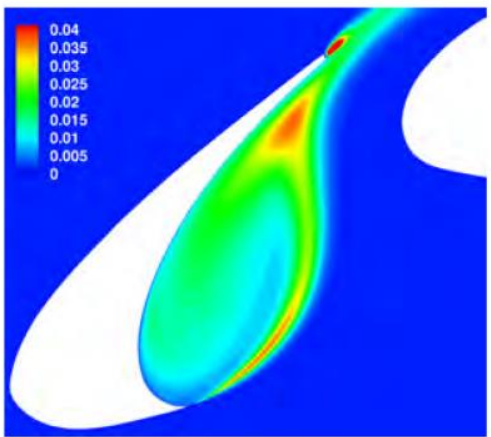

(c) 03

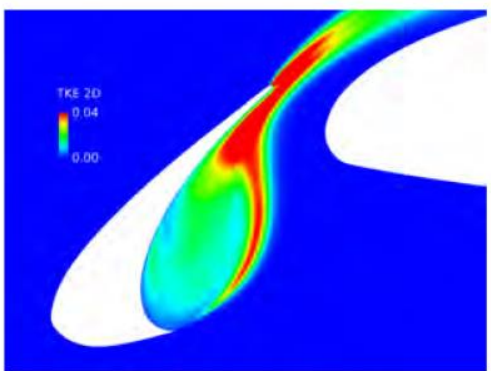

(f) 06

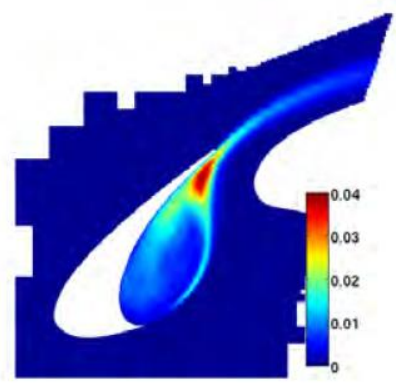

(i) 09

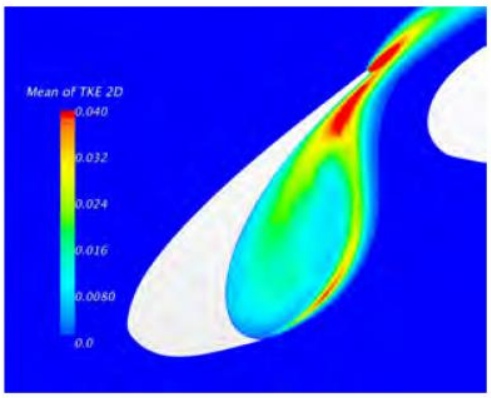

(l) 12

Figure 7. TKE $2 \mathrm{D}$ distribution in slat cove region. 


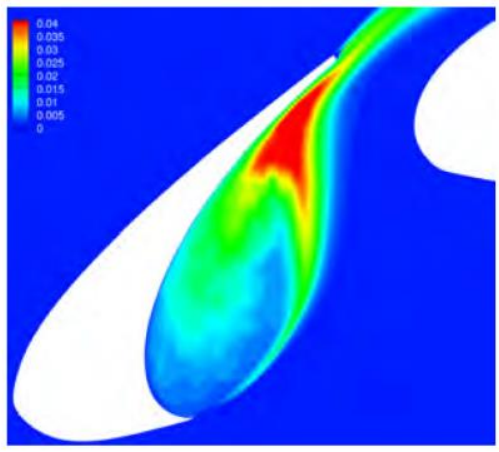

(a) 13

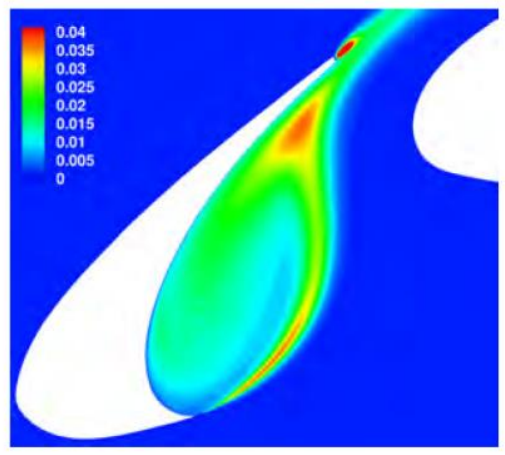

(d) 16

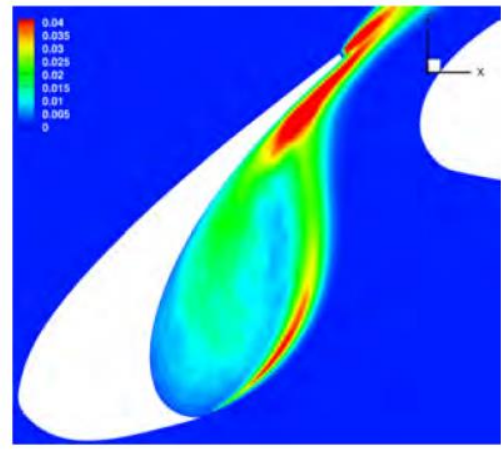

(b) 14

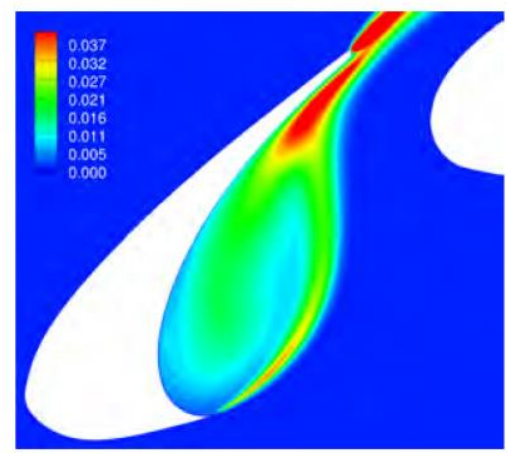

(e) 17

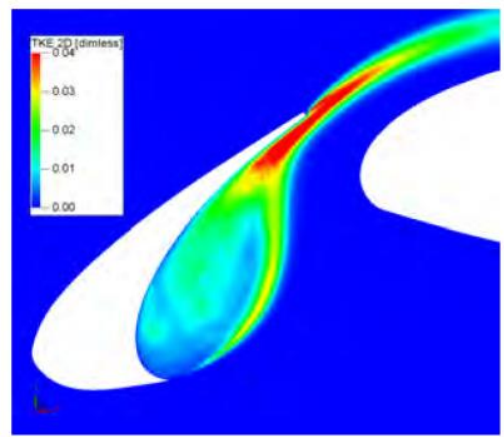

, in

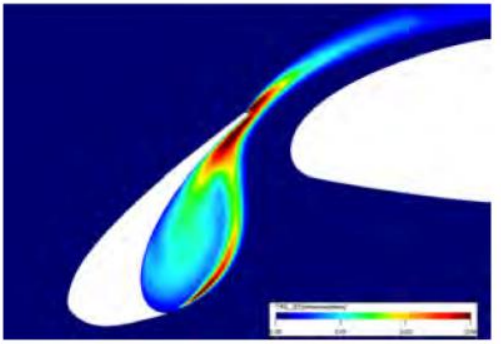

(c) 15

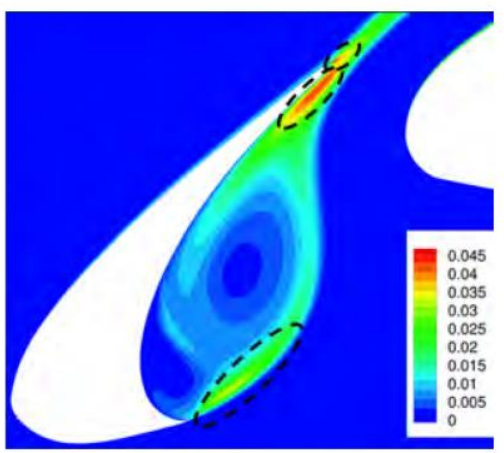

(f) 18 (RANS)

Figure 7. $\mathrm{TKE}_{2 \mathrm{D}}$ distribution in slat cove region. 


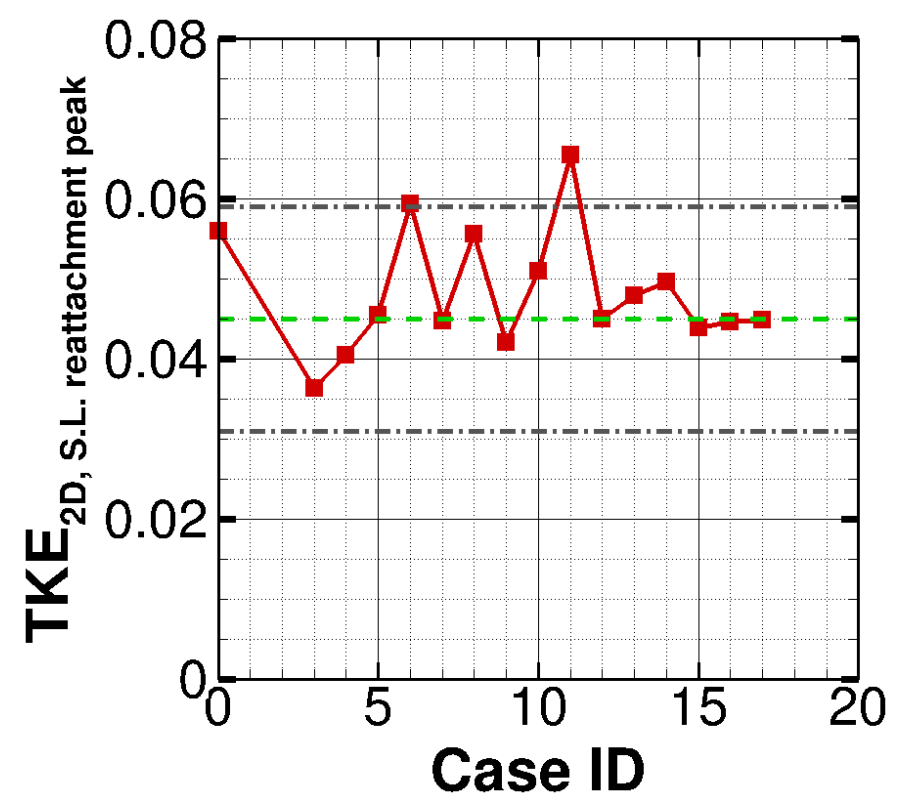

Figure 8. Peak TKE2D near shear layer reattachment. The green dashed line indicates the median of the entire set of submissions, and the two dash-dot lines bound a confidence interval.

\section{Shear Layer Profiles}

Shear layer profiles depicting both mean flow and turbulence statistics across the shear layer at selected locations along its trajectory from the slat cusp (profile L1 in Fig. 9(a)) to just upstream of the reattachment location (profile L7) were requested from the Category 7 participants. Illustrative comparisons of $\mathrm{TKE}_{2 \mathrm{D}}$ profiles for L2, L4, and L7 are shown in Fig. 9(b). The dispersion of peak $x / c$ locations among the blue curves for L7 is reflective of the significant variation in the shear layer reattachment location, which lies in the vicinity of the unsteady pressure probe locations P4 and P5 from Fig. 9(a).

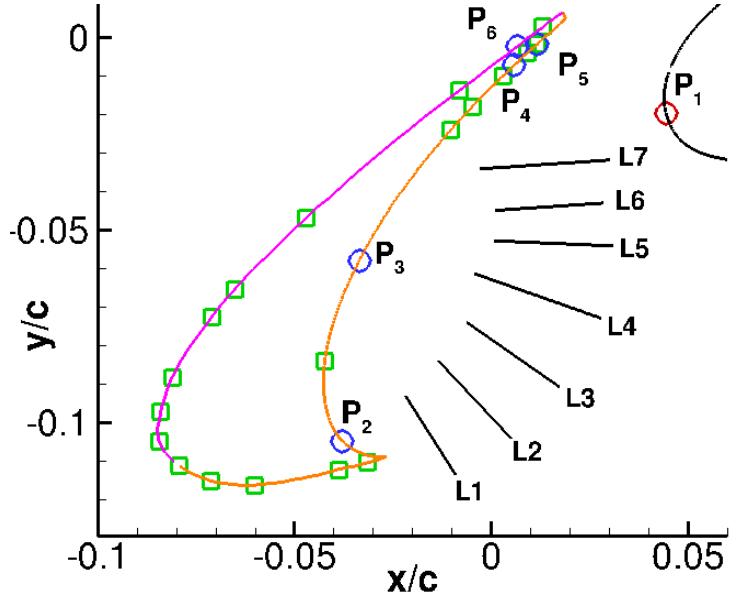

(a) Schematic of probe (P1 through P6) and profile (L1 through L7) locations. Small squares not labeled indicate static pressure ports on slat surface

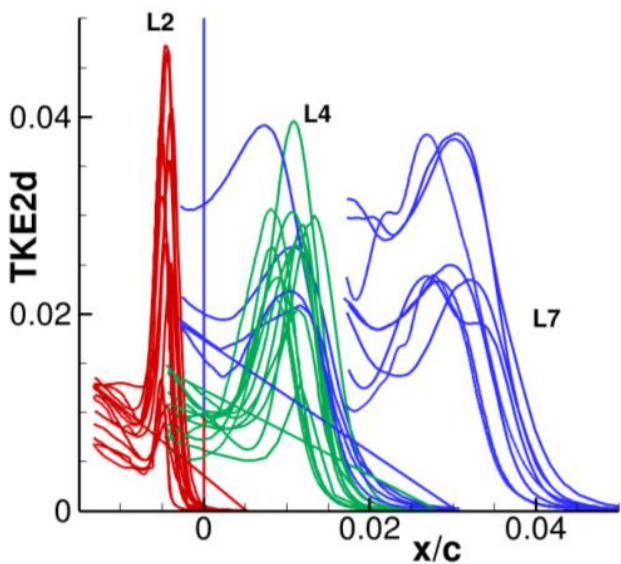

(b) $\mathbf{T K E}_{2 \mathrm{D}}$ profiles from contributed datasets for L2 (red), L4 (green), and L7 (blue) segments from the schematic in part (a)

Figure 9. Comparison of shear layer profiles 


\section{E. Mean and RMS Surface Pressure Distribution}

Consistent with the $\mathrm{C}_{\mathrm{L}}$ data in Fig. 5, the mean Cp distributions excluding the outliers are also indicative of an analogous bimodal behavior. The two branches of the mean $\mathrm{Cp}$ distribution are plotted separately in Figs. 10(a) and 10(b), respectively. The differences between the two branches are reflected in the respective suction peaks over the main wing, the suction peaks and the locations of pressure plateau (i.e., apparent separation locations) over the flap, and most significantly, in the pressure levels over a majority of the suction surface of the slat. Of course, because of the inter-element coupling within the 3-element high-lift configuration, neither one (nor a selected subset) of these differences can be identified as the cause behind the remaining features. These correlated facets of the overall differences are potentially associated with the differences in turbulence model (and, secondarily, other factors such as domain size, farfield boundary condition, etc.)

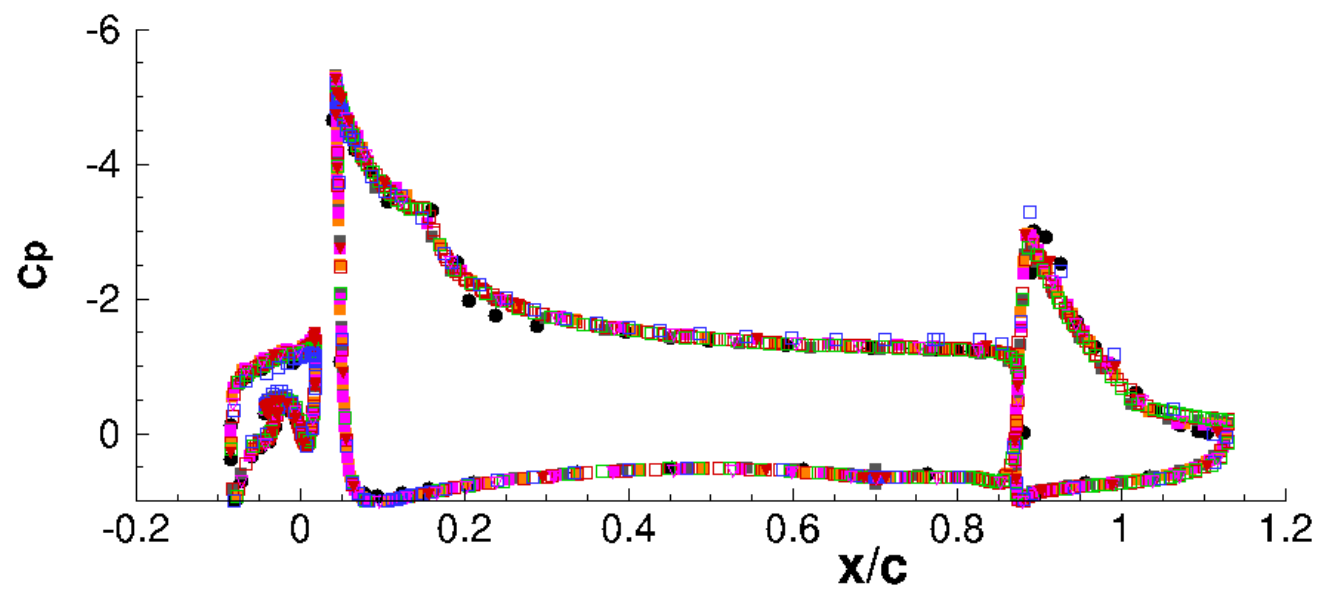

(a) Submissions 03-08, 15, and 16 compared with the FSU measurement (open symbols denote the CFD predictions, whereas filled black circles denote the measurement)

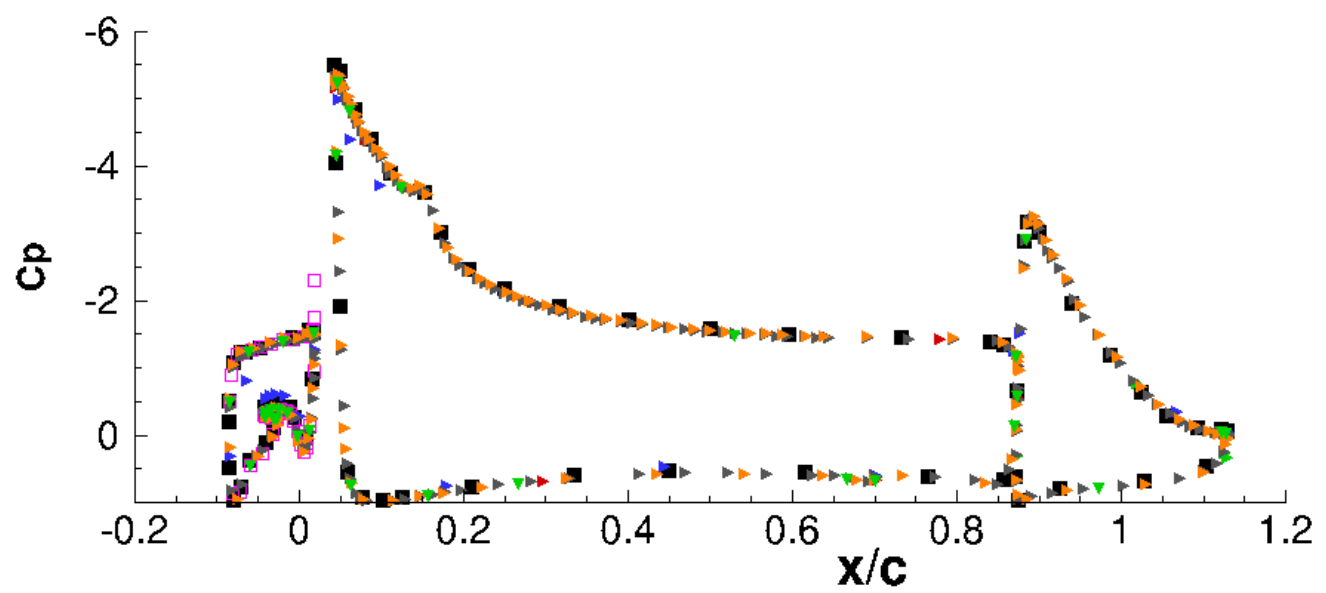

(b) Submissions 11-14 and 17 compared with the JAXA measurement (open symbols denote the CFD predictions, whereas filled black squares denote the measurement).

Figure 10. Two branches of mean Cp distribution.

The reader may note that the experiments at both $\mathrm{FSU}^{19}$ and $\mathrm{JAXA}^{20}$ were performed in closed-wall wind tunnels with significant end wall effects that make it difficult to determine an effective angle of attack 
independently of comparison with open-air, 2-D (or short-span) CFD computations. During both sets of measurements (which were obtained immediately before the BANC-III Workshop), the "equivalent" free-air angle of attack for the high-lift configuration in these facilities was identified via comparisons with separate CFD solutions that represent the two different lobes of the bimodal $\mathrm{Cp}$ distribution. Hence, the measured $\mathrm{Cp}$ distributions agree well with the respective branch of the CFD solutions. Of course, this discrepancy can be rectified in principle by using a common reference to determine the "equivalent" free-air angle of attack during future measurements. The ability to reconcile such differences represents a positive aspect of the long-term, ongoing interplay between the computations and experiments related to this category.

A comparison between the RMS Cp' distributions over all 3 elements of the airfoil surface is shown in Fig. 11(a). A zoomed in view of this figure emphasizing the RMS $\mathrm{Cp}^{\prime}$ distribution over the slat and the main wing leading edge is shown in Fig. 11(b). The highest pressure fluctuations over the slat surface are known to occur near the reattachment location of the slat cove shear layer as well as near the trailing edge. Additional peaks are present near the main wing leading edge and farther downstream (including near the reattachment location in the cove region near the wing trailing edge) and also over the flap where the flow separates from the suction surface. The peak over the slat surface is of most interest for Category 7. An analysis of the contributed results shows that, with a fixed flow solver, the RMS $\mathrm{Cp}^{\prime}$ near the reattachment location decreases in magnitude when the underlying spatial grid is refined. For example, within the CFL3D group of solutions (i.e., cases 3 to 5), the peak levels for the coarse grid case (Submission 03) are approximately 37 percent higher than those in the fine grid Submission 05. There is considerable variability in the RMS $\mathrm{Cp}^{\prime}$ distributions over the main wing and the flap. For example, the zonal RANS treatment for the flap region in Submissions 03 to 05 leads to a considerable reduction in the level of resolved $\mathrm{Cp}^{\prime}$ fluctuations over the flap relative the other, non-zonal simulations. Submissions 13 and 14 show a strong peak on the main wing suction surface near $x / c=0.25$. The reason for such peaks should be investigated to gain greater insights into the dominant unsteady flow phenomena resolved in a given simulation.

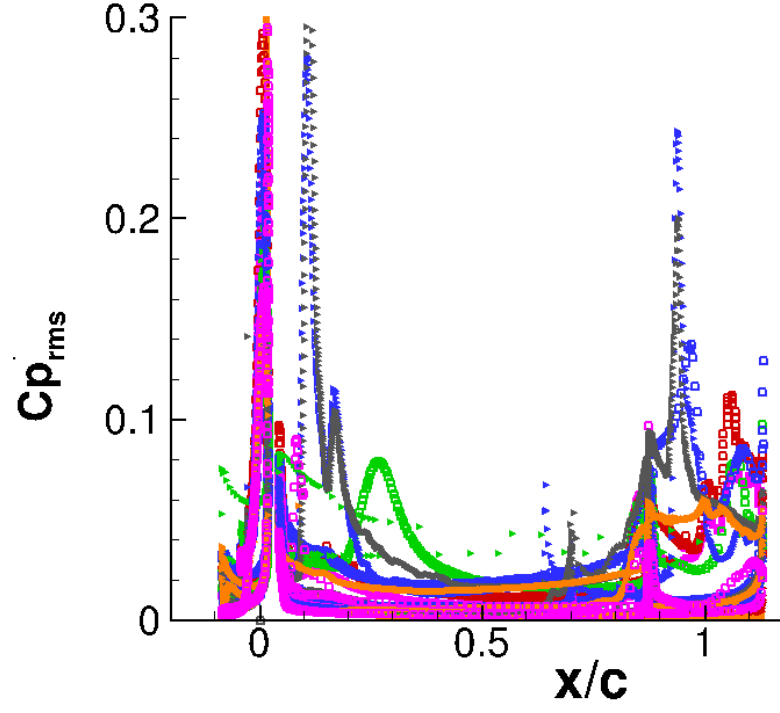

(a) Overall view including all 3 elements.

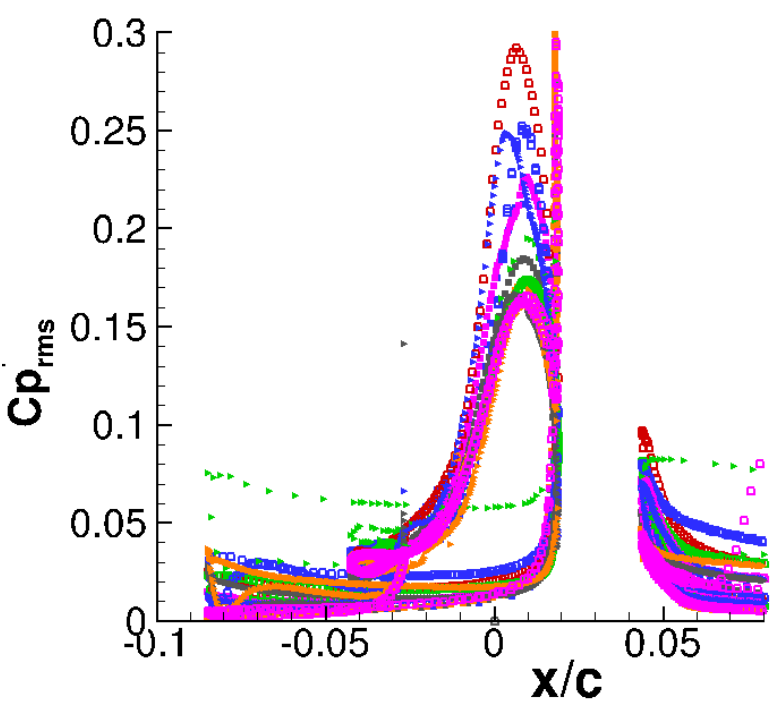

(b) Zoomed in view near slat and main-wing leading edge.

Figure 11. Comparison of RMS Cp' distribution

\section{F. Reattachment Location on Pressure Surface of the Slat}

As previously mentioned, the high intensity fluctuations in the vicinity of the shear layer reattachment (i.e., impingement) location are likely to play an important role in slat noise. Indeed, the slat noise levels (and, especially, the narrow-band peaks) are known to be sensitive to the AoA. There are two significant effects of the change in AoA on the slat flow. First, the stagnation point on the slat shifts closer to the cusp when the AoA is increased; and, second, the shear layer impingement location shifts away from the slat trailing 
edge and the gap region. Both of these effects are likely to contribute to lower slat-noise levels observed at higher AoA. Thus, a measure of the proximity between the source location (i.e., point of shear layer impingement) and a potential catalyst underlying the partial conversion of hydrodynamic energy to acoustics (i.e., slat trailing edge or the slat-main wing gap) is likely to be useful in interpreting the results. A comparison of the distance between the impingement location and the lower corner of slat trailing edge (normalized by the stowed chord length $c$ ) is plotted in Fig. 12. The two cases with lowest values of impingement distance correspond to Submission 09 (which falls outside of the confidence interval) and Submission 02 (which falls just inside the confidence interval). These low values may be attributed to the preliminary nature of these solutions, and they should approach the other solutions when the grid resolution is improved. Unlike $\mathrm{C}_{\mathrm{L}}$, there does not appear to be a significant correlation between the turbulence model and the impingement distance. The higher values of distance for Submissions 13, 14, and 17 are consistent with the generally higher $C_{L}$ values for the SA model. On the other hand, the impingement distance for Submission 11, which also had a $C_{L}$ value that is commensurate with the above SA model solution, is now comparable to corresponding values for the group with SST model. Submissions 06 and 07, which defied the trend in $\mathrm{C}_{\mathrm{L}}$ with changes in turbulence model, again predict reattachment distances that are closer to the SST group in spite of using the SA model themselves. Overall, the predicted impingement distances from a number of solutions are clustered near the median value of 0.0165 , within the narrow range of $(0.015$, 0.017). This range is significantly lower than the measured distance of approximately 0.02 inferred from the SPIV measurements in the FSU tunnel. However, the specific impingement point is difficult to discern from the SPIV measurements because strong laser reflections corrupt the image pair correlation close to the slat surface. Future experiments will attempt to resolve this issue to obtain reliable data closer to the surface for a more accurate approximation. In the region of steeper gradients in the RMS $\mathrm{Cp}^{\prime}$ distribution along the slat surface, depending on the particular simulation, a distance of $0.035 \mathrm{c}$ can amount to a reduction in surface SPL of as much as $2.7 \mathrm{~dB}$ just inside of the impingement location (i.e., on the cusp side) and $4.8 \mathrm{~dB}$ on the trailing edge side. Of course, the sensitivity of SPL to surface location is significantly reduced at the impingement location itself, because of the local maximum in $\mathrm{RMS} \mathrm{Cp}^{\prime}$ distribution. The sparse measurements based on surface pressure transducers do not allow this local maximum to be measured; however, there are prospects of alleviating that shortcoming via unsteady PSP measurements as reported in the JAXA experiments. ${ }^{20}$ The impingement distance for Submissions 13 and 14 lie just above and below the upper boundary of the confidence interval; however, this may not be significant in light of the measured value being larger than the median. Overall, there is good agreement between the FSU measurement and the impingement distances for Submissions 14, 16, and 17; however, it must be viewed in the context of the holistic comparison as remarked in the Introduction.

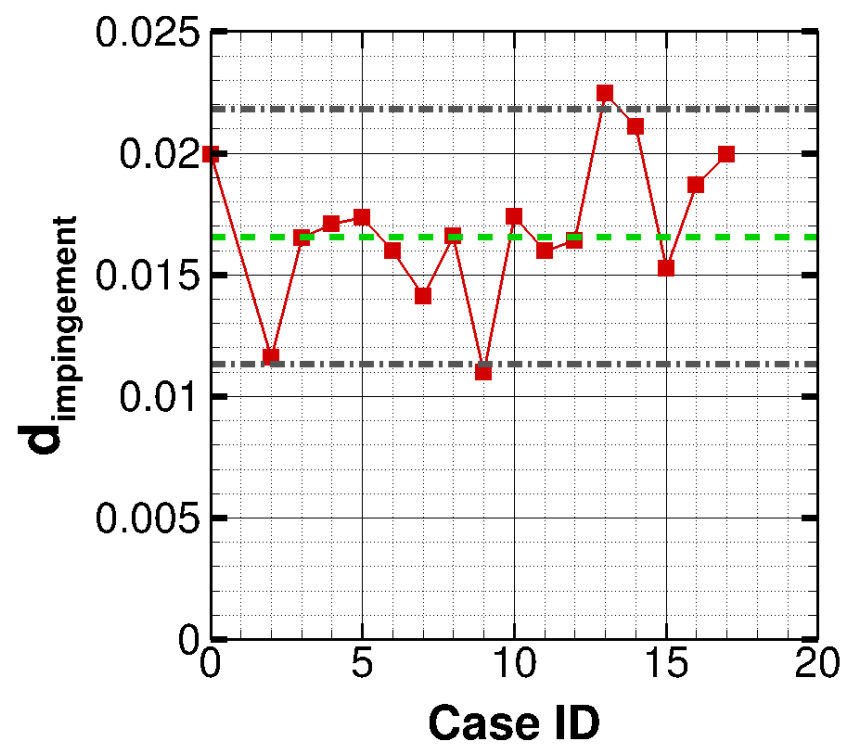

Figure 12. Distance between shear layer impingement location and lower corner of slat trailing edge. The green dashed line indicates the median of the entire set of submissions, and the two dash-dot lines bound a confidence interval. 


\section{G. Unsteady Surface Pressure Spectra}

Three different probe locations from the schematic in Fig. 8 are used to illustrate the comparison of nearfield spectra based on surface pressure measurements. Figures 13 through 15 display the results for probe locations P1, P4, and P6, respectively The probe location P4 is near the reattachment location of the slat cove shear layer and, hence, is dominated by hydrodynamic fluctuations associated with the impingement of the shear layer on the solid surface. The other two locations are not directly exposed to hydrodynamic fluctuations and primarily represent the acoustic component of the unsteady surface pressure, i.e., they may be viewed as representative (and easier to measure) metrics of the nearfield acoustics associated with this configuration. Consequently, the spectral levels at P1 and P6 are more than $20 \mathrm{~dB}$ lower than those at $\mathrm{P} 4$. The relatively intense broadband hydrodynamic fluctuations at $\mathrm{P} 4$ also tend to mask the underlying narrowband peaks, which are more clearly observed in the frequency spectra at the P1 and P6 probe locations. The submission of data for the P6 probe location was optional. Hence, there were only three entries that provided this spectrum and those are plotted in Fig. 15.

The P4 spectrum was measured in both the FSU and JAXA wind tunnels and, somewhat surprisingly, exhibit an $\mathrm{O}(5) \mathrm{dB}$ difference in their PSD amplitudes. Also, the NBPs in the FSU spectrum (Submission 00) are better defined (i.e., sharper and attaining higher PSD values) than those in the JAXA spectrum. The underlying shape of the broadband spectrum is quite similar across the full range of solutions, including the (likely) underresolved Submission 02, which is unable to predict any NBPs and has PSD values that are nearly $8-10 \mathrm{~dB}$ higher than even those in the JAXA measurement. The P4 spectrum based on the finer of the two grids used for the simulations with the LAVA code matches the lower PSD values in the FSU measurement pretty well up to approximately $2.2 \mathrm{kHz}$ (i.e., past the second prominent NBP in the portion of FSU spectrum included in this figure), but has levels at higher frequencies that are closer to those of the P4 spectrum measured in the JAXA facility. The NBP frequencies are somewhat different from those in the FSU spectrum as well. The spectra for Submissions 07 and 11 are somewhat similar up to and including the second NBP and are not greatly different from the Submission 14 in this region, except for rather intense NBPs in comparison with both the Submission 14 and both measured spectra. The Submission 07 spectrum exhibits an earlier onset of rapid, high-frequency roll off, which could be an indication of coarser grid resolution. The ostensibly underresolved simulation of Submission 09 appears to have a reasonable broadband floor through $2.2 \mathrm{kHz}$, but has an overly strong NBP near $2 \mathrm{kHz}$ and the spectrum rolls off at lower frequencies than the spectrum for Submission 07. The spectrum for Submission 12 compares favorably with the JAXA measurement through $3.5 \mathrm{kHz}$, but its slower roll-off leads to higher PSD values at the higher frequencies. The Submission 15 spectrum falls roughly in between the two measurements up to $4 \mathrm{kHz}$, i.e., over a major portion of the slat cove noise, but is closer to the JAXA measurement for frequencies larger than $2.5 \mathrm{kHz}$. Of the three different grids used with the CFL3D solver, the coarse grid spectrum (Submission 03) matches the JAXA measurement quite well through $4 \mathrm{kHz}$ and then rolls off rapidly. Improving the grid resolution delays the rolloff until $f \approx 7 \mathrm{kHz}$ for Submission 04; however, with an additional two-fold increase in the grid resolution (i.e., Submission 05), the PSD levels become slightly smaller than the JAXA measurement up to $3 \mathrm{kHz}$, but they remain moderately above the measurement from $4 \mathrm{kHz}$ up to $10 \mathrm{kHz}$. Submission 06 yields a P4 spectrum that is relatively close to the Submission 05 result and may actually be slightly closer to the JAXA measurement. Overall, the predicted P4 spectra in Submissions 05, 08, 10, 15, 16, and 17 are similar to each other, with PSD variations of O(3) $\mathrm{dB}$ or less.

To some degree, the aforementioned comparison is influenced by the differences in the probe location relative the peak of RMS Cp' distribution along the slat surface. Such differences can have a significant impact on the measured PSD levels as mentioned in the preceding subsection in the course of comparing the distance between the impingement location and the slat trailing edge. Again, global measurements providing information about the RMS $\mathrm{Cp}^{\prime}$ levels and associated frequency spectra over a denser set of probe locations would be useful in reconciling the discrepancy in PSD levels at probe location P4.

The comparison for the nearfield acoustic spectra at probe location P1 is somewhat similar to that involving the primarily hydrodynamic spectra at probe location P4. Specifically, the spectral shapes are again similar. Submission 02 shows PSD levels that are 10-20 dB higher than those in the JAXA measurement up to $3 \mathrm{kHz}$, and the spectrum does not show any evidence of NBPs. The spectral shape for Submission 12 is closer to that of the JAXA measurement across the entire range of frequencies, but the 
levels are consistently 10-20 dB too high. A discrepancy of this magnitude in an otherwise well resolved simulation would suggest a scaling error. However, that appears less likely since the spectrum plotted here already corresponds to a revised submission after fixing a similar error. Submissions 09, 11, and 7 show more intense peaks at NBP frequencies than the other predictions or the measurement. Predictions of Submissions 03, 04, and 08 agree fairly well with each other and the measured spectrum, except for a noticeable overprediction of PSD values at frequencies less than approximately $1.2 \mathrm{kHz}$. Submission 08 also shows stronger NBPs than the measured spectrum at frequencies beyond $3.5 \mathrm{kHz}$. The latter feature is also shared by the P1 spectra in Submissions 05, 06, and 15, which have somewhat better agreement with the measured spectrum at the lower frequencies (less than $1.2 \mathrm{kHz}$ as noted above for Submissions 03, 04, and 08) but also display a valley region (i.e., reduced PSDs) between approximately $2.8 \mathrm{kHz}$ to $4 \mathrm{kHz}$. The overall agreement between the JAXA measurement of the P1 spectrum and those predicted in Submissions 05, 06, and 15 is quite good, and possibly better than the agreement seen for probe location P4. Because the P4 spectrum involves sound propagation from the slat cove region to the main wing leading edge, the predicted spectra may be influenced by the narrow spanwise width of the domain, but perhaps not by much in view of the relative proximity of those two regions. The spanwise width may play a greater role with increasing distance from the slat cove region. The inclusion of more distant surface probe locations, such as along the nose of the slat and/or on the pressure surface of the main wing, may be useful.

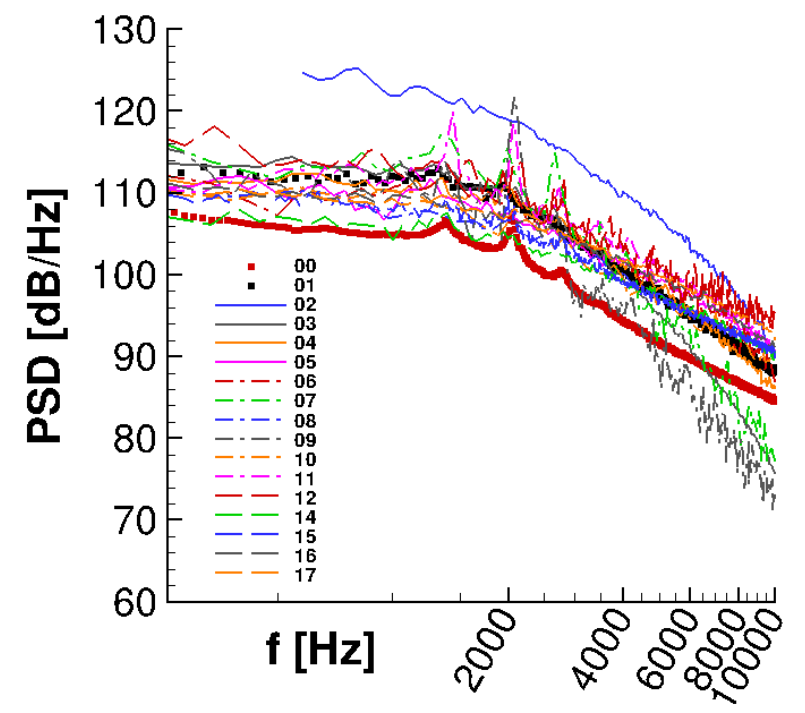

Figure 13. Frequency spectra of surface pressure fluctuations at probe location P4.

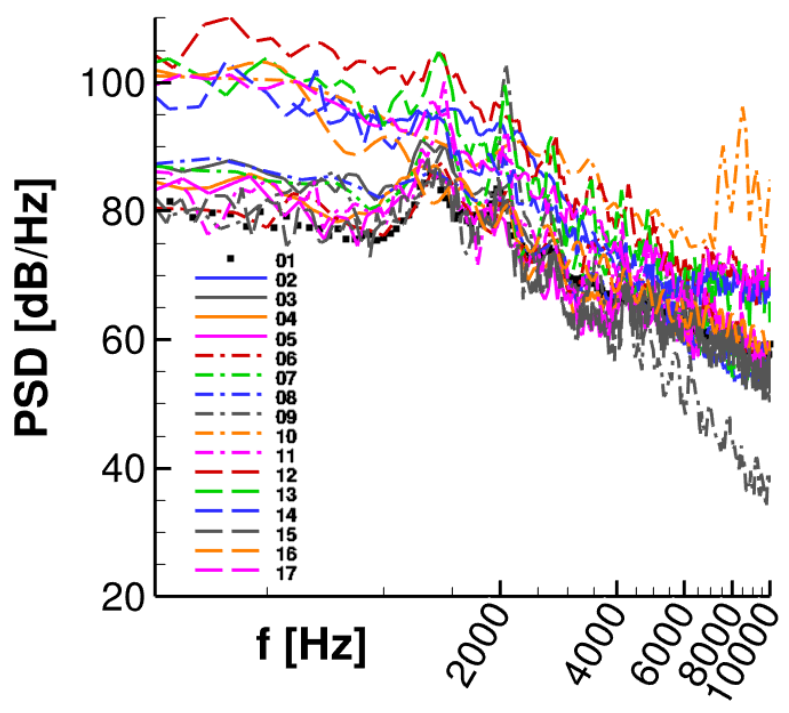

Figure 14. Frequency spectra of surface pressure fluctuations at probe location P1.

All three spectra for the P6 probe location agree well in shape. The predicted spectrum for Submission 12 agrees with the measured spectrum in the FSU facility in both shape and the PSD levels, except for the presence of an extra NBP near $1.6 \mathrm{kHz}$. The physical origin of the extra NBP is not clear at this stage. On the other hand, the predicted spectrum for Submission 15 does not have the extra peak, but the PSD values are 3-5 dB below the other two spectra. These differences may be, in part, due to the sensitivity to probe location as discussed in the context of the P4 probe location. If so, choosing the P6 probe location in close proximity of the trailing edge may have been somewhat overambitious; and, in future work, additional comparisons farther away from the trailing edge (in a flatter region of the RMS Cp' distribution) may be more meaningful. 


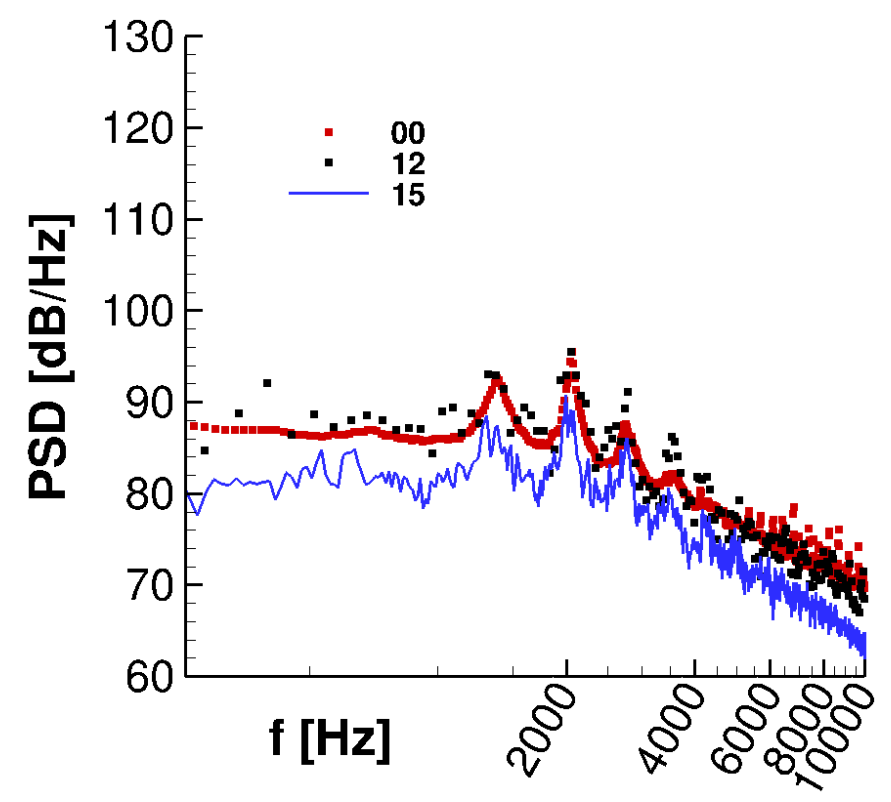

Figure 15. Frequency spectra of surface pressure fluctuations at probe location P6.

The available farfield acoustic data from microphone array measurements in the JAXA LWT2 facility cannot be directly used for a quantitative comparison with the predictions based on narrow-span simulations. As a substitute, finding consistent trends among the comparisons involving the nearfield acoustic spectra would make it easier to extrapolate from the nearfield spectra to those of the farfield acoustics. However, the limited comparison involving location P6 indicates somewhat different trends from those discussed earlier for the nearfield spectra at P1. Such differences would make any inferences concerning farfield acoustics on the basis of nearfield acoustics spectra less definitive if not meaningless.

\section{H. Spanwise Coherence of Unsteady Surface Pressures}

The spanwise coherence of unsteady surface pressures is an important factor in accurate predictions of farfield acoustic spectra. An example of the dependence of the coherence on the AoA observed in the JAXA experiment is shown in Fig. 16. Only a limited subset of contributors provided this data, possibly because it requires more involved postprocessing than the simpler auto-spectrum calculation. Coherence is not an easy quantity to compute (and to compare in this particular case) because of the mixed nature of the underlying frequency spectrum and the small number of time series segments permissible within the duration of a typical BANC-III computation. Although not shown, the narrow-span computations show a high degree of spanwise coherence in the nearfield acoustics at probe location P1. There is encouraging agreement in the spanwise coherence at $\mathrm{P} 4$, but there are considerable quantitative differences that are worth investigating during follow-on computations.

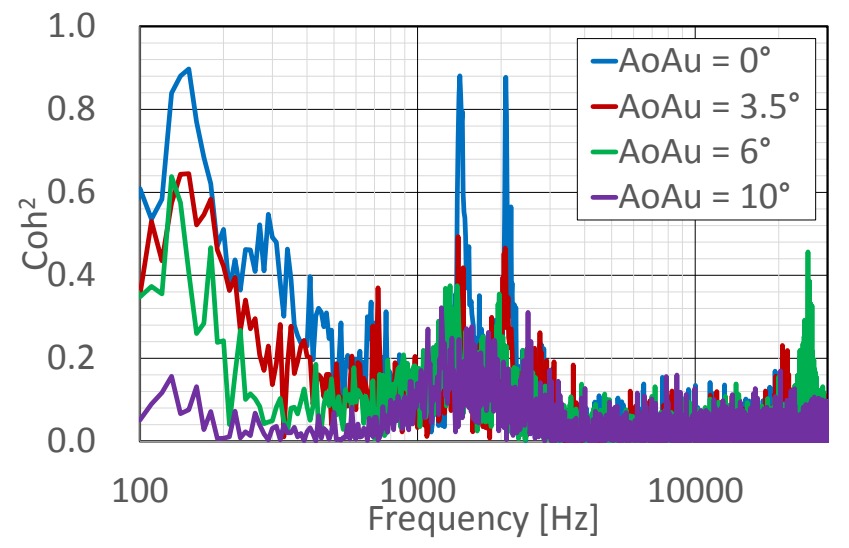

Figure 16. Spanwise coherence of surface pressure fluctuations at P4 (Submission 01) ${ }^{20}$. 


\section{Acoustic Spectra Including OASPL at Selected Probe Locations}

The predicted sound pressure levels at an overhead observer location (i.e., 270 deg azimuthal location in Fig. 17(a)) ten chord lengths away from the origin are shown in Fig. 17(b). The predictions in Fig. 17(b) have been band filtered to the frequency range between $(256 \mathrm{~Hz}, 10 \mathrm{kHz})$ in an attempt to remove the contributions from trailing edge shedding, separated flow over the flap, and in general, high amplitude low frequency disturbances whose statistics are not adequately converged within the limited time duration of the numerical simulations. For Submission 16, the frequency range was limited to $(256 \mathrm{~Hz}, 7.4 \mathrm{kHz})$ to avoid an instability that eventually overwhelmed the solution. Because of the influence of the spanwise width of the computational domain on the farfield acoustic spectra, ${ }^{46}$ the participants were asked to normalize the predictions to a span of $0.0254 \mathrm{~m}$ (i.e., 1 inch). In some cases, this was accomplished by limiting the spanwise width of the FWH data surface to this value and, in a few others, a coherence length of less than the spanwise domain width was assumed to scale the predictions. Based on the findings below and the fact that most participants in Category 7 were able to manage a domain width of $0.0508 \mathrm{~m}$, an updated Category 7 problem statement should prescribe the spanwise width for both the $\mathrm{FWH}^{47}$ data surface and the CFD domain, leaving the effects of the width to be studied as an optional exercise.

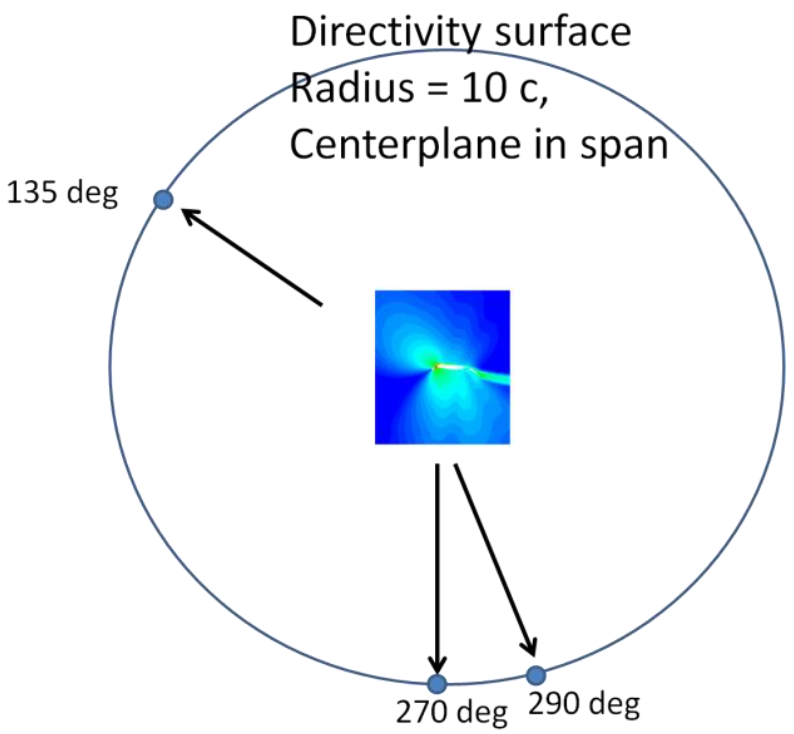

(a) Schematic of directivity surface and acoustic observer locations.

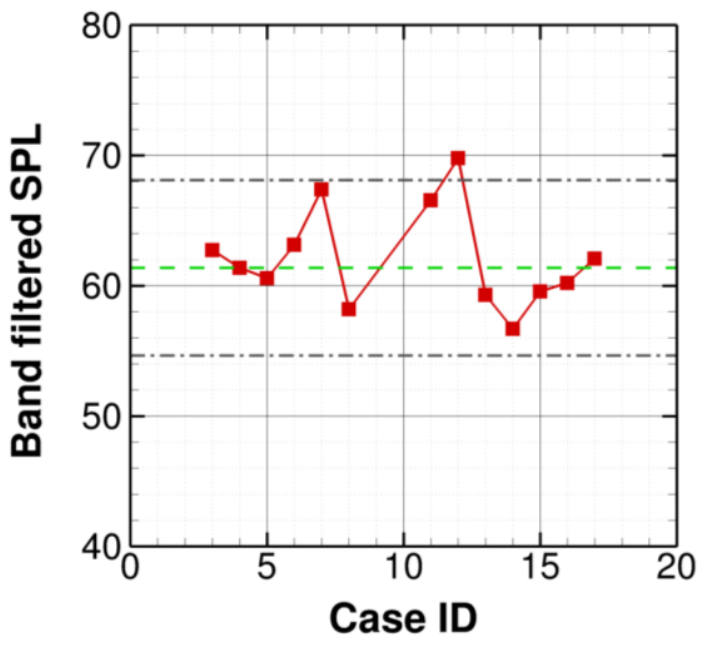

(b) Band filtered SPL (dB) at 270 deg observer location from the schematic in part (a).

Figure 17. Comparison of farfield acoustic amplitudes (SPL). The green dashed line indicates the median of the entire set of submissions, and the two dash-dot lines bound a confidence interval.

The frequency spectra at the same distance but for a neighboring observer location of 290 deg orientation are shown in Fig. 18. Spectra for Submissions 07, 11, and 12 indicate rather intense NBPs, consistent with the trend in the nearfield acoustic spectra. The acoustic spectra for Submissions 13 and 14 correspond to lower PSD values than the other submissions, but also indicate moderately strong NBPs at high frequencies near $7 \mathrm{kHz}$. The remaining spectra (Submissions 03, 04, 05, 06, 08, 15, 16, and 17) are relatively similar, with the exception of a few differences such as additional NBPs below $1 \mathrm{kHz}$ in Submissions 05 and 15 that were not observed in the surface pressure spectra. Furthermore, the farfield acoustic spectrum of Submission 16 exhibits a very strong NBP near $7.7 \mathrm{kHz}$ that approximately corresponds to a planar waveguide mode (associated with acoustic propagation in the computational domain, i.e., an empty "test section" with periodic boundary conditions) such that its wavelength is equal to the spanwise width of the computational domain. ${ }^{35,36}$ Analogous peaks that may also be related to the spanwise domain width are seen at $f>6 \mathrm{kHz}$ frequencies in a few other Submssions such as 03,04, 05, 13, 14, and 17. The contributions from these peaks have influenced the comparison of band-pass SPLs in Fig. 17(b). The low pass limit of 
this band was set to filter out the anticipated frequency of vortex shedding from the slat trailing edge; however, a reduced limit of around $5 \mathrm{kHz}$ may be more appropriate during future comparisons of band filtered SPL. The intense waveguide mode in Submission 16 would have resulted in the farfield SPL in Fig. 17(b) being significantly larger than that of other submissions with relatively similar PSD values at lower frequencies, so the upper limit for the band-pass filter was reduced to $7.4 \mathrm{kHz}$. The strength (and even the very presence) of this waveguide mode appears to be a strong function of the flow solver and the degree of artificial dissipation associated with the numerical scheme. The occurrence of this waveguide resonance introduces an interesting quirk in regard to the effect of spanwise domain width on the fidelity of the simulation. In general, one expects virtually monotonic increase in the fidelity as the domain width is increased. However, that is not necessarily the case in the present range of spanwise domain widths. For example, had the spanwise domain width been larger by a factor of 3 , the waveguide mode would have surfaced right in the vicinity of the NBPs associated with the physical part of the spectrum. This is another reason why it is important to examine the sensitivity of the simulation results to the spanwise domain width.

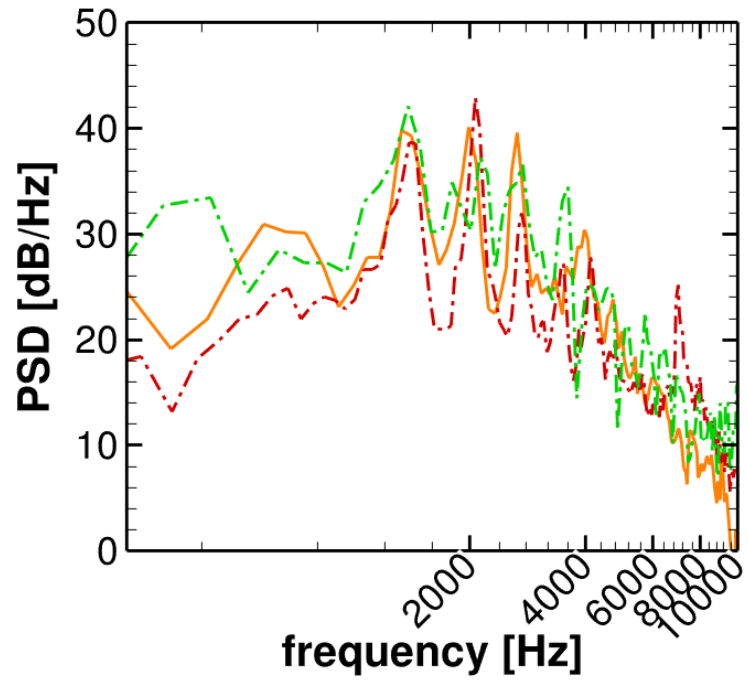

(a) Submissions 12, 7, 11.

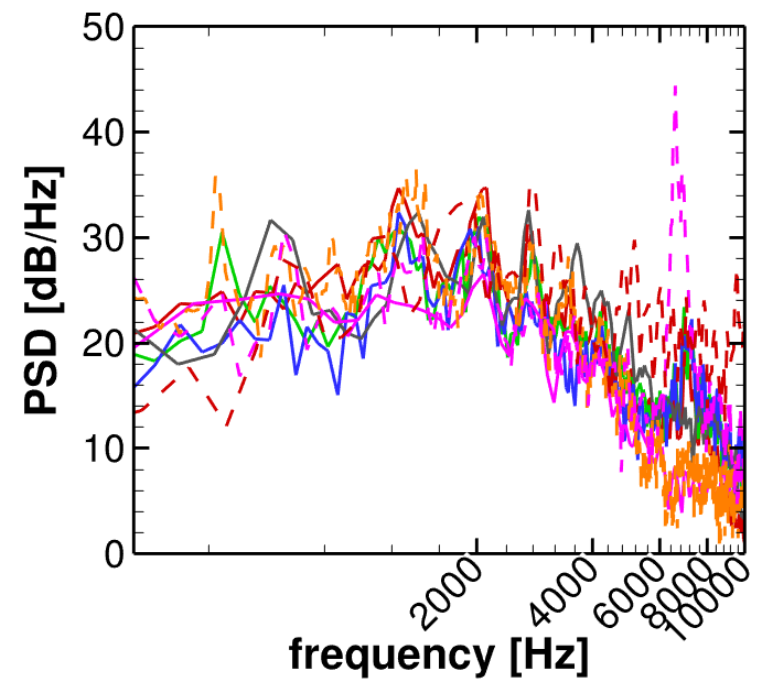

(b) Submissions 03, 04, 05, 06, 08, 15, 16, 17.

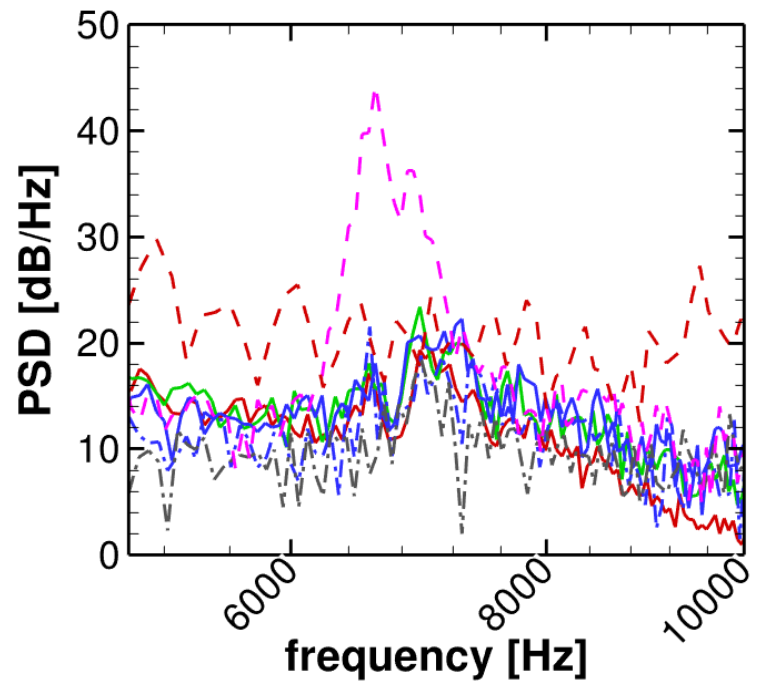

(c) Submissions 03, 04, 05, 13, 14, 16, 17.

Figure 18. Farfield acoustic spectra at 290 deg observer location from Fig. 17(a). 
The acoustic spectra analyzed above were obtained using solid surface data from the nearfield solution. Only one participant (Submission 11) provided acoustic predictions based on nearfield data on both the solid surface and a permeable $\mathrm{FWH}^{47}$ surface, and they showed good agreement between the two predictions, indicating that the corrections due to quadrupole contributions from off-body turbulent eddies are modest, at best, for the present slat noise configuration.

\section{J. Acoustic Directivity}

A comparison of the computationally predicted broadband directivity patterns (band filtered to $(256 \mathrm{~Hz}$, $10 \mathrm{kHz}$ ) range to focus on slat cove noise) is shown in Fig. 19. To allow an assessment of the directivity pattern separately from the underlying values of the OASPL (which was plotted in Fig. 17(b)), the different curves have been normalized by their respective values measured at the overhead position (i.e., 270 deg location in Fig. 17(a)).

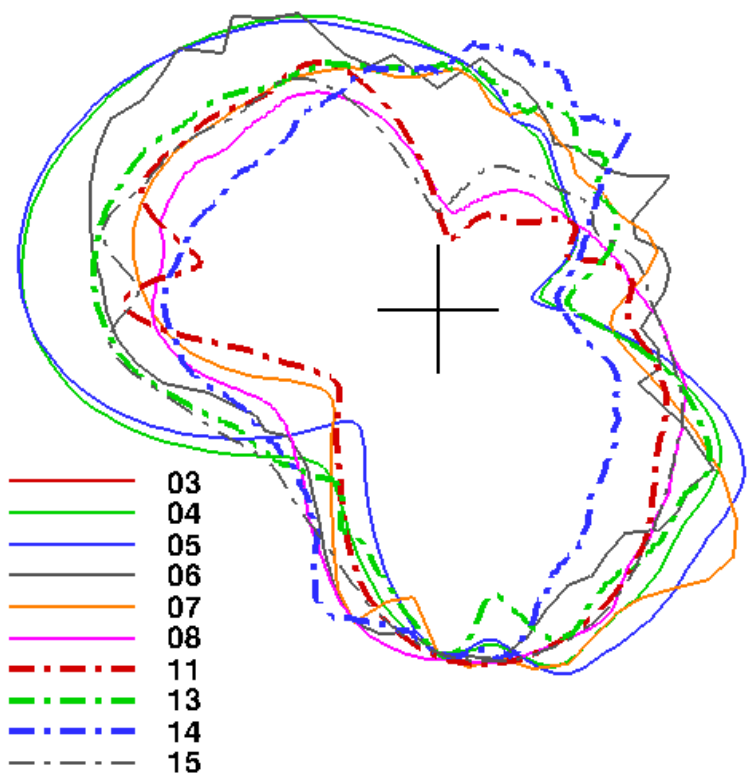

Figure 19. Band filtered $(256 \mathrm{~Hz}$ to $10 \mathrm{kHz})$ directivity patterns normalized to overhead observer location.

With the exception of Submission 16 (not shown), the broadband directivity in the $x y$ plane is similar in all datasets, and is reminiscent of the directivity of a dipole as originally suggested by Dobrzynski and PottPollenske $^{48}$ on the basis of experimental measurements. Thus, there are two main peaks and two clear valleys in the directivity pattern. Similar to the directivity pattern of a dipole, the valley regions are approximately opposite to each other (i.e., displaced by $180 \mathrm{deg}$ ). However, such is not the case for the relative orientations of the two peak regions. While the peak within the lower half plane is located within the rear arc, the main peak within the forward sector of the upper half plane corresponds to a smaller inclination with the horizontal direction. Both peaks are relatively broad and their precise directions vary somewhat from one solution to another. A number of solutions also exhibit additional secondary peaks (presumably indicative of the interference pattern caused by reflections off the 3-element airfoil surface) including a modest peak within the rear arc of the upper half plane. The overall scatter in directivity levels near the lower lobe appears to be weaker than in the forward direction (which is also of less practical significance as an annoyance metric for obvious reasons).

The band-filtered SPL values at the overhead observer location (270 deg) in Fig. 17(b) indicate a spread of $10 \mathrm{~dB}$ after discounting the outlier (Submission 12) and the coarse grid result from Submission 07 for which a prediction based on a finer grid is available (Submission 06). However, besides Submission 11 (which, again, corresponds to a coarser grid than some others) and Submission 14 (which yields the lowest 
noise level among the set), all remaining predictions are clustered within a $\pm 3 \mathrm{~dB}$ band. Such differences are probably comparable to the uncertainties introduced by the different (and rather modest) values of the span of the computational domains. The predicted acoustic quantities are the outcome of a complex set of phenomena associated with the nearfield flow solution, the acoustic filter intrinsic to the simulation process, and the acoustic analogy solver that identifies the filtered acoustic component of the nearfield solution and propagates it to the far field. Since the contributed solutions are expected to show differences in each of these factors, the differences found above are not surprising. However, the dominant cause of the observed differences in OASPL levels (Fig. 17(b)) and/or the directivity patterns (Fig. 19) cannot be easily pinpointed.

\section{K. Narrow-band peaks (NBPs) in Nearfield and Acoustic Spectra}

As mentioned in the Submission Description (section II), slat cove aeroacoustics associated with nominally 2-D high-lift configurations at subscale Reynolds numbers is known to exhibit NBPs superimposed on an otherwise broadband frequency spectrum. The NBP frequencies based on a spectral analysis of the unsteady surface pressure and farfield acoustics are shown in Fig. 20. The relative intensity of the NBPs strongly depends on the observer location and the participants were allowed to use their own criteria to determine the peak frequencies. A majority of them used the surface pressure spectrum at probe location P1 on the main leading edge, whereas a few of them also used the acoustic spectrum in addition to the surface pressure spectrum. One participant used the surface pressure spectrum at probe locations P2 and P3.

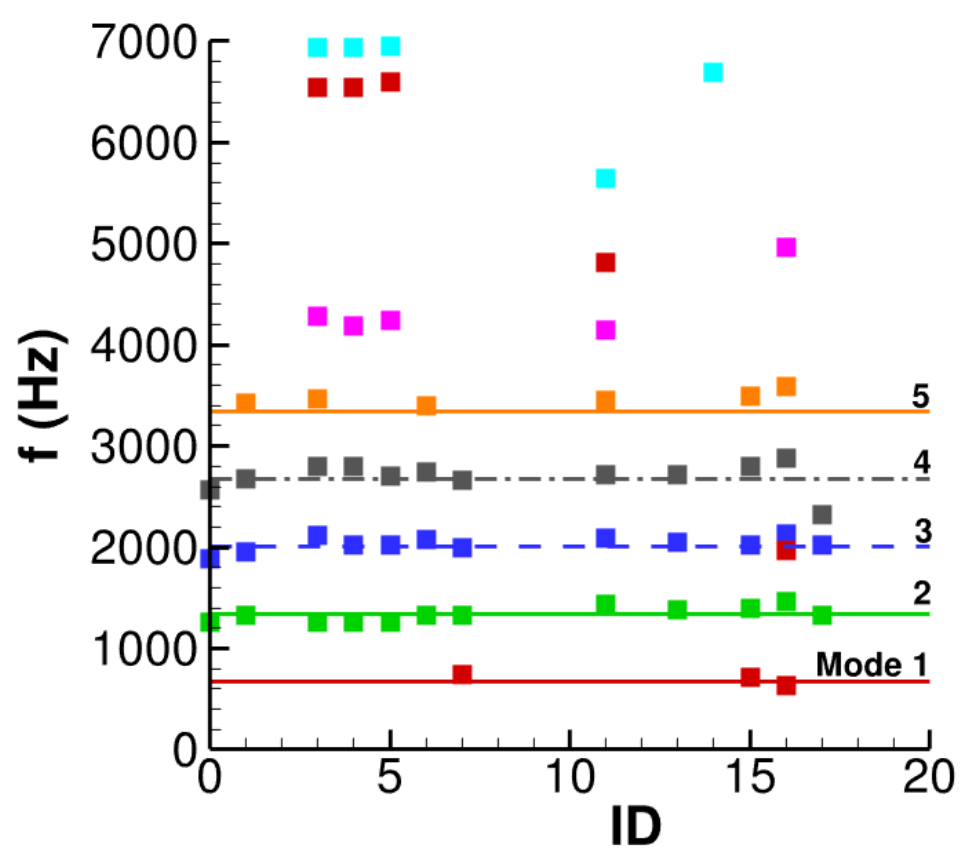

Figure 20. Frequencies corresponding to NBPs

Recent information from the literature ${ }^{49-52}$ supports the idea that physical mechanisms analogous to the Rossiter ${ }^{53}$ modes of a rectangular, open cavity are also responsible for the slat-cove NBPs. Several models have been proposed to predict the frequencies of feedback tones associated with rectangular open cavities in a flat surface. Typically, these models require the phase change across the feedback loop (i.e., the argument of the complex open loop gain) to be an integer multiple of $2 \pi$, which leads to an infinite sequence of NBPs corresponding to frequencies of $f_{n}=(n+C) f_{1}$, where $n=1,2, \ldots$ and $0<C<1$. In a number of low-speed cavity configurations, $C$ is sufficiently small so that the sequence of NBPs reduces to approximately integer multiples of the fundamental frequency $f_{1}$. Despite recent attempts ${ }^{49-52}$ to apply cavity resonance models to slat-cove NBPs with appropriately selected parameters of the slat-cove flow field, we note that due to the increased geometric complexity and the presence of multiple feedback paths, simple cavity models are not 
likely to provide accurate quantitative predictions for NBP frequencies for a broad class of high-lift configurations. Rather than estimating the model constants with heuristic arguments that may or may not be entirely valid, we adopt a pragmatic approach to anchor the comparison of NBPs. Instead of predicting the fundamental mode frequency on the basis of geometry and flow parameters for the high-lift configuration, we simply fit the abovementioned empirical expression to the measured data under the assumption that $C=$ 0 , based on the findings of the FSU experiment. ${ }^{19}$ The resulting fits are shown as NBP modes 1 through 5 in Fig. 20.

Due to the stochastic nature of the PSD estimates and the modest number of averages underlying the spectrum evaluation (especially when using the limited time history from the computations), the expected uncertainty in the estimated NBP frequencies is significantly greater than one half of the bin width of the discrete spectral estimates. In light of this uncertainty, the agreement among the various simulations and the experimental data is rather striking. Indeed, the agreement on the scale of Fig. 20 extends across all contributors who provided NBP data for comparison. Mode 1 has not surfaced in a majority of the computations. Due to the lower amplitude of the mode 1 peak in cases where it was present, the type of spectrum used to determine the NBPs may have played a role in this mode not being picked in the other simulations. For Submissions 03, 04, and 05, the mesh resolution has not had a significant impact on the frequency of mode 2 , which actually corresponds to the first peak (i.e., lowest frequency) in the computed spectra because of mode 1 not being observed in those simulations.

\section{Grid Sensitivity Studies}

Establishment of grid converged aeroacoustic predictions for airframe noise configurations has been an elusive goal to date, especially for the problems addressed under the BANC Workshops that are pushing the state of the art in terms of the difficulty of the underlying problem and, hence, stretching the limits of easily available computational resources. In view of this fact, seeing that multiple participants made an attempt to examine the sensitivity of the requested solution metrics to grid resolution is gratifying. After setting aside the preliminary attempts (Submissions 02, 09, and 10) and considering the simulations in Submissions 11, 12, and 17 as a single group in view of their sharing a common family of grids (as well as noting that the Univ. of Manchester group also performed computations on an unstructured grid but did not have long enough output samples to compute reliable statistics), we observe that a majority of contributors in Table 1 provided solutions on more than a single grid. For the simulations that involved just two grids, significant overall differences have been observed between the two predictions as noted in the previous subsections. However, the community has recognized the importance of this task and is making some inroads into establishing the sensitivity of the different metrics to the grid resolution, at least for the grid sizes that are currently feasible for these problems in the context of BANC related efforts. A few encouraging trends may be noted from the CFL3D computations employing two levels of a factor-of-2 grid refinement (Submissions 03, 04, and 05), and also from Submission 15 simulations at 3 different grid levels.

The overall findings from the results described in previous subsections indicate that the peak TKE levels tend to increase with grid resolution, but the RMS pressure fluctuations decrease on the finer grids. The effect of grid resolution is most apparent in the level of NBPs, which become less prominent on the finer grids. The simulations from Submission 15 demonstrate that results for Category 7 metrics that are relatively insensitive to the grid resolution can be achieved within the limitations of the underlying computational methodology (i.e., LBM with a Cartesian octree grid and surface elements). This finding should be interpreted rather loosely and at a pragmatic level, as it does not mean that grid converged predictions have been obtained for every single metric. The boundary layer resolution remains rather limited and there is considerable scope for further grid refinement before the ostensibly VLES results would asymptote to the level of a DNS prediction.

As an illustration of the relative insensitivity to the grid resolution, Fig. 21 shows the nearfield acoustic spectra at probe location P6, obtained with the baseline grid (denoted as the fine grid) and a grid that is 1.5 times coarser (the medium grid). Shear layer profiles on these two grids, along with those on a third grid that is coarser than the medium by a factor of 2, are shown in Fig. 22. The $\mathrm{TKE}_{2 \mathrm{D}}$ shows a modest sensitivity to grid refinement, especially for the L2 and L4 profiles; however, the mean velocity and even the vorticity profiles are nearly the same between the medium and the fine grid simulations. A comparison of the instantaneous vorticity contours (Fig. 23) indicates that the flow structures continue to develop fine scales in the process of grid refinement, but those scales have a relatively weak effect on the metrics shown in Figs. 21 and 22. 


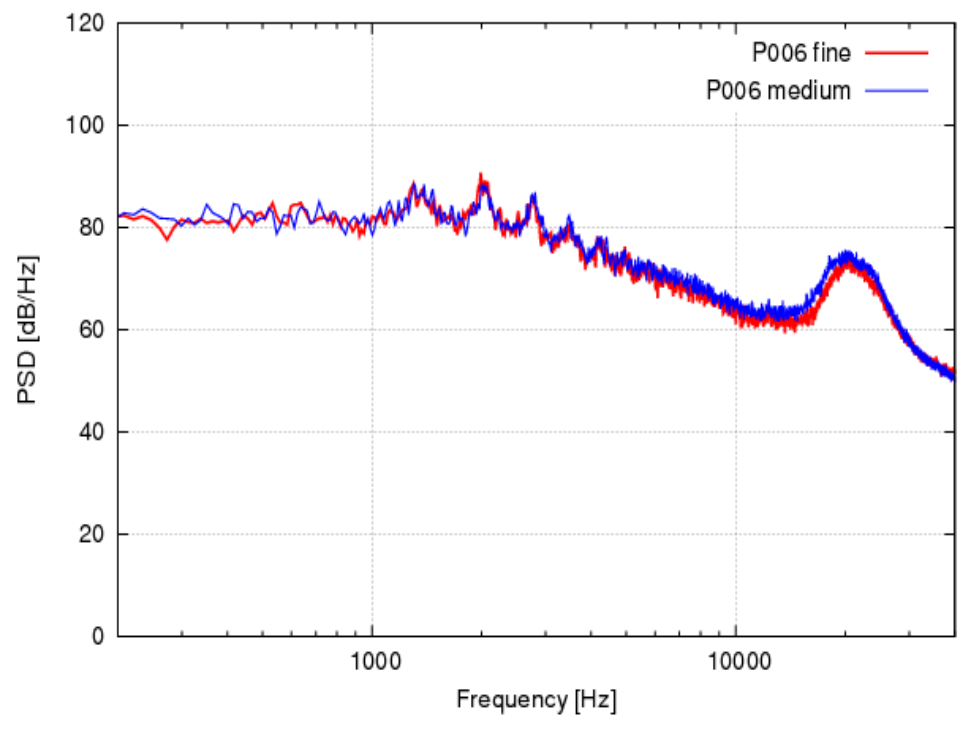

Figure 21. Nearfield pressure spectrum at probe location $\mathbf{P 6}$ for a family of successively refined grids (Submission 15).

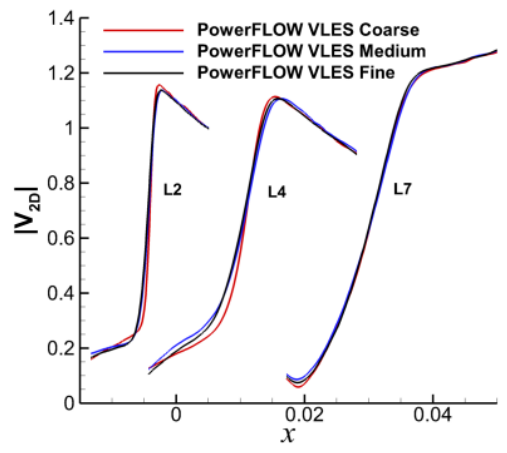

(a) Velocity magnitude

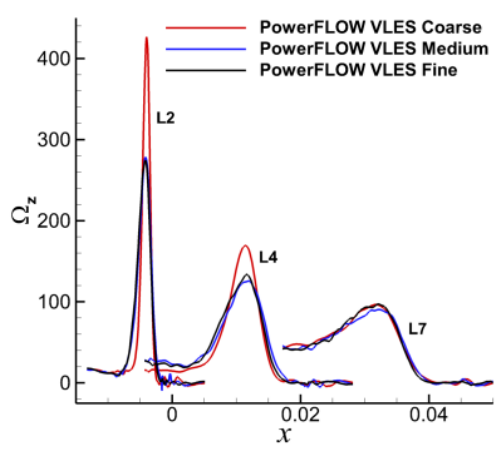

(b) Spanwise vorticity.

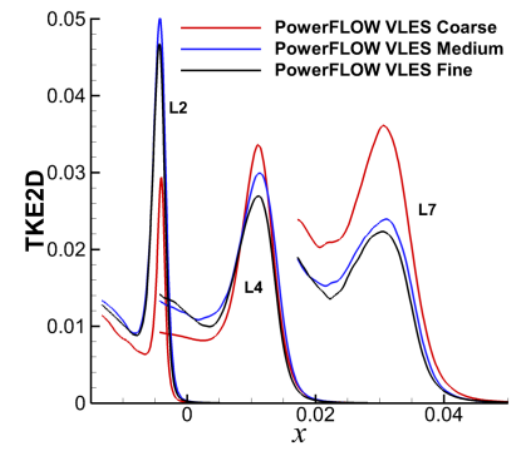

(c) TKE $2 \mathrm{D}$.

Figure 22. Shear layer profiles for a family of successively refined grids (Submission 15).

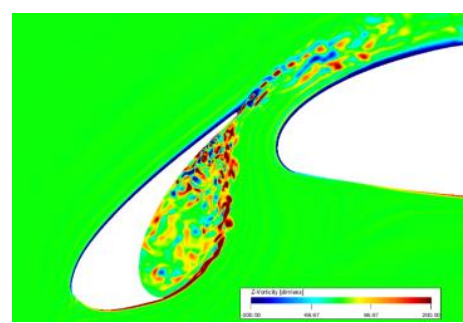

(a) Coarse grid (grid -2).

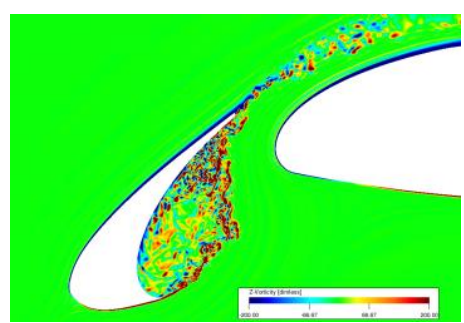

(b) Medium grid (grid -1).

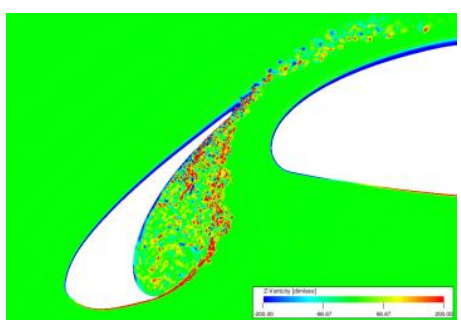

(c) Fine grid (grid 0).

Figure 23. Contours of instantaneous spanwise vorticity for a family of successively refined grids (Submission 15).

\section{Open Ended Component of Category 7 Problem Statement}

The Category 7 problem statement included an open ended component that suggested several options for supplementary simulations that would contribute to the technical objectives of this category. The optional computations were intended to help fill in the gaps that could not have been addressed on a common basis, 
such as more in depth studies of grid convergence, effects of spanwise domain width, flow Reynolds number, angle of attack, as well as end-wall effects in wind tunnel experiments. Additionally, participants were encouraged to analyze the rich database provided by their numerical simulations so as to improve the understanding of noise generation mechanisms related to slat-cove noise (Fig. 1). Given the requirements of performing the simulations and the required elements of post-processing, the expectations concerning the optional add-ons were rather low at this stage. Nonetheless, a few teams rose to the challenge and contributed useful insights that are summarized below in the hope of encouraging a deeper analysis during future efforts. The details of the analysis underlying these findings are deferred to future publications by the individual participants.

Analysis of the simulation database by team 08 allowed them to confirm the suspected role of fluctuations near the shear layer impingement location as a dominant contributor to slat cove noise. Extra computations accompanying Submission 19 (which was the sole contribution based on a reduced-order model for the slat-cove noise) provided further insights into the noise generation process by indicating two separate physical mechanisms with comparable contributions to the farfield acoustics. These two mechanisms include: a scattering of hydrodynamic fluctuations near the slat trailing edge and the acceleration of unsteady flow structures through the gap between the slat trailing edge and the main-wing leading edge.

The grid refinement study in Submissions 03 to 05 revealed an apparent discrepancy between the resolved frequencies in the near- and farfield. Although deviations could be observed in the nearfield spectra at a frequency $f_{l}$, the farfield spectra were in good agreement until a much higher frequency $f_{2} \sim 5 f_{1}$. They conjectured that the nonlinear nature of noise generation and the large difference in wavelength between hydrodynamic and acoustic waves in low speed flows could explain the observations.

Supplementary postprocessing by team 15 provided further details of the flow structures and acoustic radiation patterns associated with the NBPs. As an example, Fig. 24 reveals how the number of vortex wavelengths along the shear layer trajectory increases by one across each successively higher NBP. The lowest NBP (which actually corresponds to mode 2 from Fig. 20) leads to a dipole-like acoustic radiation pattern (Fig. 25(a)), but the peak radiation angle in the upper half plane shifts increasingly towards the forward direction as the NBP frequency increases (Fig. 25(b) through 25(d)). Higher-order spectral analysis of the unsteady pressure history near the reattachment location was used to assess the possibility of nonlinear interactions between the unsteady flow structures associated with the NBPs. Bicoherence peaks in Fig. 26 indicate a weak but statistically significant interaction between the multiple narrow-band peaks.

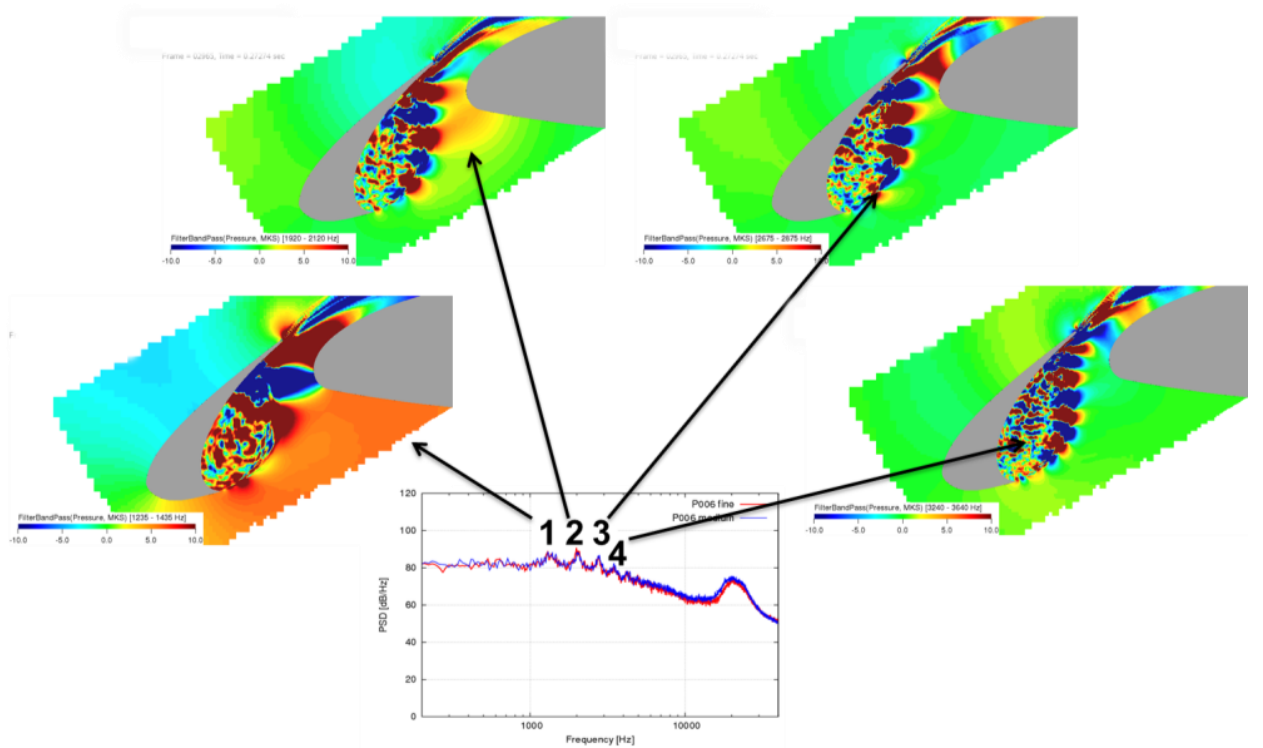

Figure 24. Band filtered nearfield pressure distribution corresponding to NBPs. 


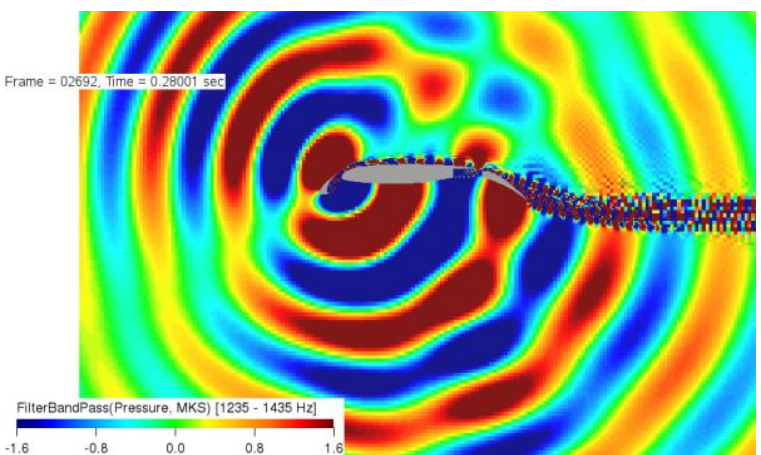

(a) NBP 1.

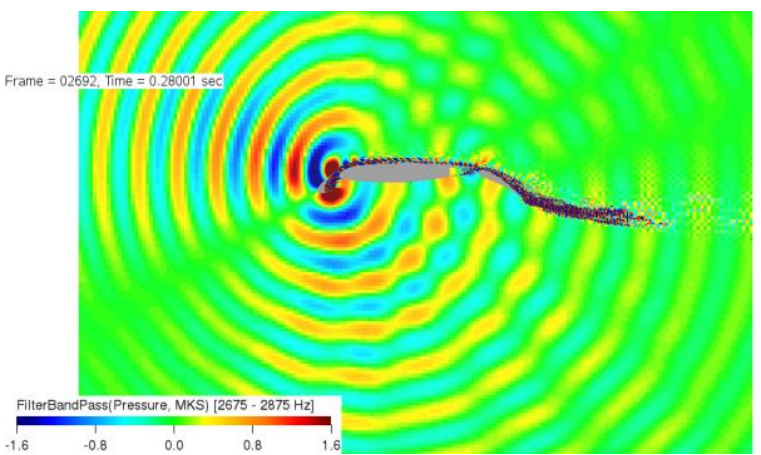

(c) NBP 3.

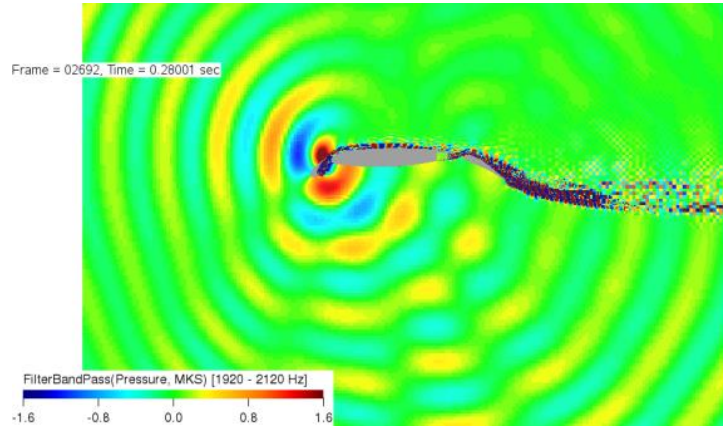

(b) NBP 2.

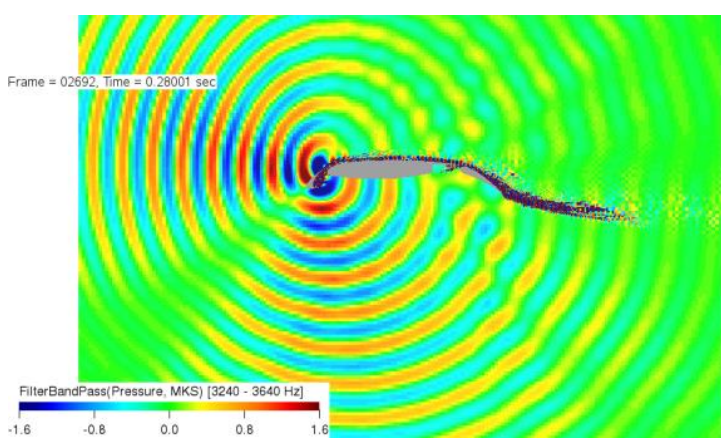

(d) NBP 4.

Figure 25. Band filtered acoustic radiation patterns associated with NBPs 1 through 4 from Fig. 24.

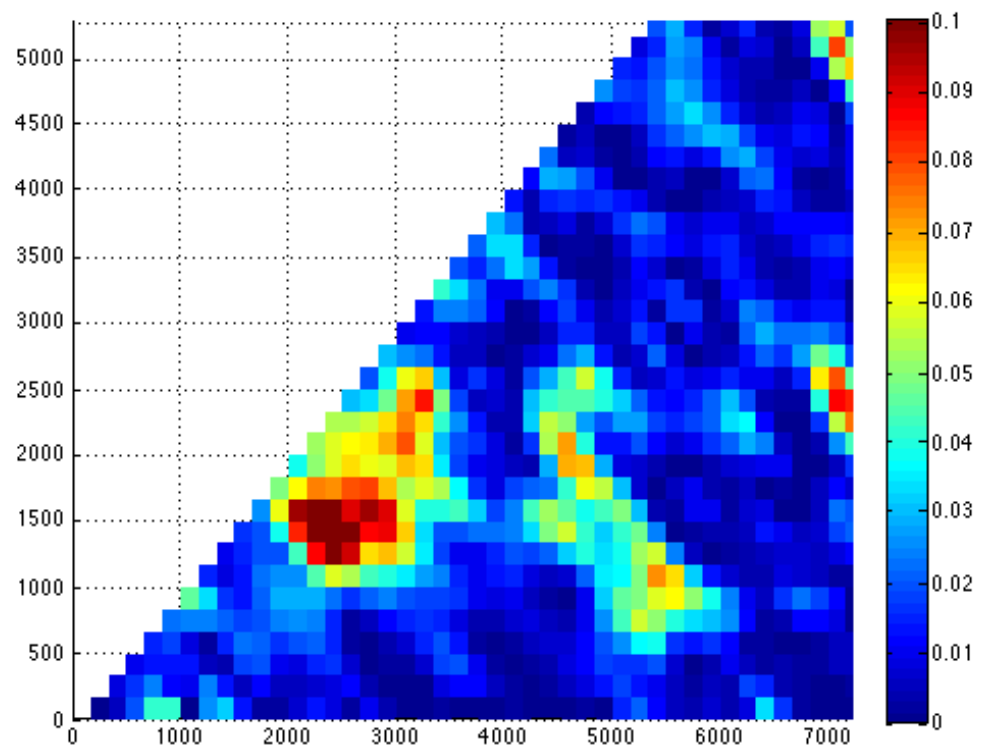

Figure 26. Bicoherence peaks in pressure signal at reattachment location. 


\section{Summary and Concluding Remarks}

Category 7 of the BANC-III Workshop focused on the slat-cove aeroacoustics of the generic, unswept 30P30N 3-element high-lift configuration with slightly modified slat contour. The experimental contributions to this category provided mean and unsteady surface pressures, limited surface flow visualization, off-body velocity field measurements using stereoscopic particle image velocimentry, and microphone-array based acoustic measurements in a conventional facility. Nearly twenty computational solutions covered a broad spectrum of methodologies in terms of numerical algorithms (finite-volume, second-order and higher-order finite-difference, and the lattice Boltzmann method), turbulence modeling (large eddy simulations (LES), hybrid Reynolds averaged Navier-Stokes (RANS)-LES, and delayed detached eddy simulations (DDES)), and meshing techniques (structured grid with contiguous, patched, or overset interfaces between adjacent blocks, Cartesian meshes coupled with immersed boundary techniques or surface elements, and unstructured grids). One of the contributions was related to reduced-order modeling based on a stochastic source model combined with CAA propagation. Modeling contributions of this type signify a welcome addition to Category 7 , and the initial contribution during BANC-III should spur additional submissions of this type during the follow-on workshops.

This paper focused on a comparison of the computational predictions submitted by the BANC-III participants. Some of the comparisons involved solution metrics that were common to both experiments and simulations, whereas a few others were restricted to the computational datasets alone. Grid sensitivity studies presented by multiple BANC-III participants demonstrated a relatively consistent trend of reduced surface pressure fluctuations, higher levels of turbulent kinetic energy in the flow, and lower levels of both narrow band peaks and the broadband component of unsteady pressure spectra in the nearfield and farfield. The computational datasets also allowed a limited assessment of user to user differences in the context of a fixed flow solver as well as of the effects of using different flow solvers with the same computational mesh.

Specific features of a number of computational solutions on the finer grids compared reasonably well with the initial measurements from the FSU and JAXA facilities and/or with each other. However, no single solution (or a subset of solutions) could be identified as clearly superior to the remaining solutions. The differences with the measured data were to be expected in view of the challenges in slat-noise prediction (particularly the mixed, tonal and broadband character of the acoustic spectra and the quasi-2-D geometry) as well as in the measurement of slat noise, the practical limitations on the spanwise width of the computational domain, and the finite end effects in both experiments from BANC-III.

The relatively consistent findings across the contributed set of computational solutions include a likely effect of turbulence model on the mean lift and surface Cp distribution, an increase in TKE levels at higher grid resolutions, and, yet, an accompanying decrease in the RMS surface pressure fluctuations and the predicted farfield acoustic intensity. Grid resolutions had a particularly significant impact on the narrowband peaks (NBPs) that became more intense at the coarse grid resolutions. For second-order CFD discretizations and the typical span lengths used during the BANC-III contributions, an overall grid size of 10 million cells or less is not likely to yield acceptable predictions (at least in terms of allowing a fair assessment of the underlying methodology and/or the overall computational process), although certain features such as the NBP frequencies may be captured reasonably well in some of these cases.

The agreement for nearfield surface pressure spectra between the two facilities and also among the various computations indicates larger differences than those previously seen for the similarly quasi-twodimensional tandem cylinder configuration used for Category 2 of the BANC-I and BANC-II workshops. However, there is a good chance that the above difference will be narrowed by using a common (or, at least, similar) set of grids as well as a prescribed spanwise domain during future studies.

The variations in NBP amplitudes appear to be greater than those in the broadband component of the nearfield (i.e., surface pressure) and the farfield spectra. In contrast, the overall agreement between the predicted NBP frequencies is rather encouraging. Even in experiments, the limited measurements acquired at JAXA suggest that the NBP amplitudes are rather sensitive to boundary layer trips. These observations lead us to conclude that limiting the comparison to the broadband component of noise spectra may provide more robust comparisons, especially in the near term. The comparisons further indicate that the variability in the nearfield acoustic spectra is less than that in the unsteady pressure spectra near the impingement location. Therefore, additional comparisons related to the sensitivity of PSD levels to the probe location near the reattachment would be helpful.

A few noteworthy accomplishments of the Category 7 at BANC-III are: assessment of slat noise prediction using a broad set of methodologies, a promising start to investigating the sensitivity of the 
computational predictions to numerical parameters including grid resolution, and a limited initial success towards relatively grid-insensitive aeroacoustic predictions. On the experimental side, a useful start was made to lay the foundation for a more comprehensive aeroacoustic dataset in the near future.

Integration between simulations and experiments has been a critical ingredient in facilitating the BANC goal of enabling substantial collaborative advances in physics based predictions of airframe noise. Category 7 has been consistent with the holistic focus on measurements targeted in the BANC workshops, seeking an eventual characterization of the significant links between flow turbulence and the final metric of interest in the form of farfield acoustics. The multi-faceted comparison will provide increased confidence into the reliability of the simulation process as well as a better understanding of the physics of noise generation, which is critical to the development of reduced-order prediction models for design cycle applications as well as robust yet efficient noise reduction techniques. Furthermore, the simpler benchmarks such as Category 7 will provide valuable lessons for the measurement and simulation of more complex airframe noise configurations. Yet, several opportunities still remain to improve the computational and experimental methodologies and those would be addressed during the future B ANC workshops.

The contributions to the BANC-III Workshop have suggested several improvements for the follow-on BANC-IV Workshop. To enable more meaningful comparisons, the computational grids developed for BANC-III studies (along with additional grids derived from those) will be made available for future Category 7 investigations, along with stricter guidelines concerning the spanwise domain width for nearfield and farfield predictions. An additional, complementary test condition involving a larger angle of attack will be included in the problem statement to allow an assessment specifically for the broadband component of slat noise.

Unlike the previously investigated quasi-2-D configuration of tandem Cylinders, which was envisioned as a prototype for the BANC effort and, hence, a comprehensive database was acquired prior to the workshops, the Category 7 represents an effort to evolve a complex problem with group effort. As such, the experimental efforts are evolving concurrently with the computational simulations, enabling a unique platform for meaningful interactions between computations and measurements. A major focus of the future measurements with the $30 \mathrm{P} 30 \mathrm{~N}$ configuration will include an improved set of acoustic measurements in a Kevlar-wall wind tunnel and an improved strategy for processing the microphone array data for comparison with acoustic predictions from narrow-span computations. The Kevlar-wall setup has the advantage of reproducing an aerodynamic loading that is very close to that in a closed-wall test section and an acoustic farfield that can still be measured without the major effects associated with acoustic reverberation in the closed-wall test section. Additional measurements are planned that will provide unsteady PSP measurements and, potentially, direct measurements of the state of the boundary layer near the slat cusp, providing a more in-depth characterization of the effects of boundary layer tripping.

Despite the differences in computations and measurements noted in this paper, the overall set of contributions to the BANC-III workshop indicate a positive prognosis for the BANC-IV Workshop, provided that the model installation effects in the wind tunnel can be addressed satisfactorily. In closing, we hope that this summary has provided a useful baseline to evaluate future progress and that it will be useful to interested participants in building upon the existing contributions to this problem category (as well as the related Category 6 under the BANC series of workshops ${ }^{54}$ ), and also to the broader airframe noise community.

\section{Acknowledgments}

This work was performed as part of the Acoustics discipline under the Advanced Air Transportation Technologies (AATT) project of NASA's Fundamental Aeronautics Program. The authors would like to acknowledge the contributions of all Category 7 participants in the BANC-III Workshop in Atlanta, GA, during June 14-15, 2014. Particular thanks are due to the JAXA team (Drs. Murayama, Nakakita, Yamamoto, Ura, and Ito) and the personnel at Florida State University (Mr. Pascioni and Prof. Cattafesta) who performed the aerodynamic and/or aeroacoustic measurements of the 30P30N high-lift configuration. Thanks are also due to various personnel at NASA Langley who supported the experiments in the BART facility (Mr. Jenkins, Mr. Neuhart, Dr. Khorrami, Ms McGinely, Drs. Lin and Yao) and others (Mr. Weber, Mr. Cagle, Mr. Hall, and members of the NASA Langley fabrication shop) who played an important role in hardware design and fabrication of the instrumented slat model that was used for testing in the FSU facility. The authors also acknowledge Dr. Pieter Buning for his assistance in enabling an assessment of domain-size and turbulence-model effects on the mean lift coefficient. Finally, any success achieved by Category 7 of 
the BANC-III Workshop is entirely due to the dedicated efforts of the various participants (listed in Table 1 of the paper) and all of them are acknowledged as honorary coauthors of this summary report. However, the first author assumes all responsibility for the inferences drawn from the comparison presented herein as well as for any errors in processing the data provided by the participants.

\section{References}

1 Third AIAA Workshop on Benchmark Problems for Airframe Noise Computations (BANC-III), URL: https://info.aiaa.org/tac/ASG/FDTC/DG/BECAN files /BANCIII.htm [cited 15 April 2015].

21 st AIAA CFD Drag Prediction Workshop, Anaheim, CA, June 2001, URL: http://aaac.larc.nasa.gov/tsab/cfdlarc/aiaa-dpw/Workshop1/workshop1.html [cited 15 April 2015].

3 Levy, D. W., Vassberg, J. C., Wahls, R. A., Zickuhr, T., Agrawal, S., Pirzadeh, S., and Hemsch, M. J., "Summary of data from the first AIAA CFD Drag Prediction Workshop," Journal of Aircraft, 40(5):875-882, Sep-Oct 2003.

4 2nd AIAA CFD Drag Prediction Workshop, Orlando, FL, June 2003, URL: http://aaac.larc.nasa.gov/tsab/cfdlarc/aiaa-dpw/Workshop2/workshop2.html [cited 15 April 2015].

5 Laflin, K. R., Vassberg, J. C., Wahls, R. A., Morrison, J. H., Brodersen, O., Rakowitz, M., Tinoco, E. N., and Godard, J., "Summary of Data from the Second AIAA CFD Drag Prediction Workshop," Journal of Aircraft, Vol. 42, No. 5, pp. 1165-1178, 2005.

6 3rd AIAA CFD Drag Prediction Workshop, San Francisco, CA, June 2006, URL: http://aaac.larc.nasa.gov/tsab/cfdlarc/aiaa-dpw/Workshop3/workshop3.html [cited 15 April 2015].

7 Vassberg, J. C., Tinoco, E. N., Mani, M., Brodersen, O. P., Eisfeld, B., Wahls, R. A., Morrison, J. H., Zickuhr, T., Laflin, K. R., and Mavriplis, D. J., "Abridged Summary of the Third AIAA CFD Drag Prediction Workshop,” Journal of Aircraft, Vol. 45, No. 3, pp. 781-798, May-June 2008.

8 4th AIAA CFD Drag Prediction Workshop, San Antonio, TX, June 2009, URL: http://aaac.larc.nasa.gov/tsab/cfdlarc/aiaa-dpw/Workshop4/workshop4.html, [cited 15 April 2015].

9 Vassberg, J., Tinoco, E., Mani, M., Rider, B., Zickuhr, T., Levy, D., Broderson, O., Eisfeld, B., Crippa, S., Wahls, R., Morrison, J., Mavriplis, D., Murayama, M., "Summary of the Fourth AIAA CFD Drag Prediction Workshop," AIAA Paper 2010-4547, 2010.

10 Choudhari, M. and Yamamoto, K., "Integrating CFD, CAA, and Experiments towards Benchmark Datasets for Airframe Noise Problems," Proceedings of 5th Symposium on Integration CFD and Experiments in Aerodynamics (Integration 2012), October 2012.

11 Proceedings of the First AIAA Workshop on Benchmark Problems for Airframe Noise Computations (BANCI), Stockholm, Sweden, June 2010.

12 Proceedings of the Second AIAA Workshop on Benchmark Problems for Airframe Noise Computations (BANC-II), Colorado Springs, CO, June 2012.

13 Herr, M. and Kamruzzaman, M., "Benchmarking of Trailing-Edge Noise Computations|Outcome of the BANCII Workshop," AIAA-Paper 2013-2123, May 2013.

14 Lockard. D.P., "Summary of the Tandem Cylinder Solutions from the Benchmark problems for Airframe Noise Computations-I Workshop," AIAAPaper 2011-353, 2011.

15 Spalart, P. R. and Mejia, K. M., "Analysis of Experimental and Numerical Studies of the Rudimentary Landing Gear, AIAA Paper 2011-355, 2011.

16 Spalart, P. R. and Wetzel, D., "Rudimentary Landing Gear Results at the 2012 BANC-II Airframe Noise Workshop,” Int. J. Aeroacoustics, Nos.1-2, Vol. 14, 2015, pp. 193-216.

17 NASA Slat Noise Configuration, URL: https://info.aiaa.org/tac/ASG/FDTC/DG/BECAN_files_/BANCII_category 7, [cited 15 April 2015].

18 Pascioni, K., Cattafesta, L. N., and Choudhari, M. M., "An Experimental Investigation of the 30P30N MultiElement High-Lift Airfoil,” AIAA Paper 2014-3062, 2014.

19 Murayama, M., Nakakita, K., Yamamoto, K., Ura, H., Ito, Y., and Choudhari, M. M., "Experimental Study on Slat Noise from 30P30N hree-Element High-Lift Airfoil at JAXA Hard-Wall Lowspeed Wind Tunnel," AIAA Paper 2014-2080, 2014.

20 Khorrami, M. R. and Van de Ven, T., "Summary of Contributions to PDCC-NLG Category of BANC-II Workshop," Proceedings of AIAA BANC-II Workshop, Colorado Springs, CO, June 2012.

21 Manoha, E. and Caruelle, B., "Summary of the LAGOON Solutions from the Benchmark Problems for Airframe Noise Computations-III Workshop," To be presented at the $21^{\text {st }}$ AIAA/CEAS Aeroacoustics Conference, Dallas, TX, June 2015.

22 Lopes, L., Redonnet, S., Imamura, T., Ikeda, T., Zawodnyi, N., and Cunha, "Variability in the Propagation Phase of CFD-Based Noise Prediction: Summary of Results from Category 8 of the BANC-III Workshop," To be presented at the $21^{\text {st }}$ AIAA/CEAS Aeroacoustics Conference, Dallas, TX, June 2015.

23 Dobrzynski, W., "Almost 40 Years of Airframe Noise Research: What Did We Achieve?," Journal of Aircraft, Vol. 47, No. 2, 2010. 
24 Slotnick, J. P., Hannon, J. A., and Chaffin, M., "Overview of the First AIAA CFD High Lift Prediction Workshop (Invited)," AIAA Paper 2011-862, January 2011.

25 Rumsey, C. L., Slotnick, J. P., Long, M., Stuever, R. A., and Wayman, T. R., "Summary of the First AIAA CFD High-Lift Prediction Workshop," Journal of Aircraft, Vol. 48, No. 6, 2011, pp. 2068-2079.

26 Klausmeyer, S. M., Lin, J. C., Comparative Results From a CFD Challenge Over a 2D Three-Element High-Lift Airfoil," NASA TM 112858, May 1997.

27 Paschal, K., Jenkins, L., and Yao, C., "Unsteady Slat-Wake Characteristics of a High-Lift Configuration," AIAA Paper 2000-0139, January 2000.

28 Jenkins, L. N., Khorrami, M. R., and Choudhari, M. M., "Characterization of Unsteady Flow Structures Near Leading-Edge Slat: Part I. PIV Measurements,” AIAA Paper 2004-2801, 2004.

29 Khorrami, M. R., Choudhari, M. M., and Jenkins, L. N., "Characterization of Unsteady Flow Structures Near Leading-Edge Slat: Part II. 2-D Computations,” AIAA Paper 2004-2802, 2004.

30 Choudhari, M. and Khorrami, M. R., "Effect of Three-Dimensional Shear-Layer Structures on Slat Cove Unsteadiness," AIAA J., Vol. 45, No. 9, 2007, pp. 2174-2186,.

31 Lockard, D. P. and Choudhari, M., "Noise Radiation from a Leading-Edge Slat," AIAA Paper 2009-3101, 2009.

32 Lockard, D. P. and Choudhari, M., "The Effect of Cross Flow on Slat Noise," AIAA Paper 2010-3835, 2010.

33 Lockard, D. P. and Choudhari, M., "Variation of Slat Noise with Mach and Reynolds Numbers," AIAA Paper 2011-2910, 2011.

34 Choudhari, M. M., Lockard, D. P., Khorrami, M. R., and Mineck, R. E., "Slat Noise Simulations: Status and Challenges," Proceedings of Inter-Noise 2011, ed. Hiroyuki Imaizumi, Osaka, Japan, Sept. 4-7, 2011.

35 Lockard, D. P., Choudhari, M. M., and Buning, P. G., "Grid Sensitivity Study for Slat Noise Simulations," AIAA Paper 2014-2627, 2014.

36 Lockard, D. P., Choudhari, M. M., and Buning, P. G., "Influence of Spanwise Boundary Conditions on Slat Noise Simulations," To be presented at the $21^{\text {st }}$ AIAA/CEAS Aeroacoustics Conference, Dallas, TX, June 2015.

37 Third International Workshop on High-Order CFD Methods (C3.1 Turbulent flow over a multi-element airfoil), Kissimmee, Florida, January 3-4, 2015, URL: https://www.grc.nasa.gov/hiocfd/ [cited 15 April 2015].

38 C3.1 Turbulent flow over a multi-element airfoil (1/30/2011), mceze@umich.edu, URL: https://www.grc.nasa.gov/hiocfd/wp-content/uploads/sites/22/case_c3.1.pdf [cited 15 April 2015].

39 Rumsey, C., Lee-Rausch, E., and Watson, R., "Three-dimensional effects in multi-element high lift computations," Computers and Fluids, Vol. 32, No. 5, 2003, pp. 631-657.

40 Bres, G., Freed, D., Wessels, M., Noelting, S., and Perot, F., "Flow and Noise Predictions for the Tandem Cylinder Aeroacoustic Benchmark," Phys. Fluids, Vol. 24, 036101, 2012; doi: 10.1063/1.3685102.

41 Tinetti, A. F. and Dunn, M. H. , "Acoustic Simulations of an Installed Tandem Cylinder Configuration," AIAA Paper 2009-3158, 2009.

42 Redonnet, S., Lockard, D.P., Khorrami, M.R., and Choudhari, M., "CFD-CAA Coupled Calculations of a Tandem Cylinder Configuration to Assess Facility Installation Effects,” AIAA Paper 2011-2841, 2011.

43 Choudhari, M., Khorrami, M.R., Lockard, D.P., Atkins, H., and Lilley, G., "Slat Cove Noise Modeling: A Posteriori Analysis of Unsteady RANS Simulations," AIAA Paper 2002-2468, 2002.

44 Hemsch, M. J., "Statistical Analysis of CFD Solutions from the Drag Prediction Workshop," J. Aircraft, Vol. 41, No. 1, 2004, pp. 95-103.

45 Morrison, J. H., "Statistical Analysis of CFD Solutions from the Fourth AIAA Drag Prediction Workshop," AIAA Paper 2010-4673, 2010.

46 Seo, J. H., Chang, K. W., and Moon, Y. J., “Aerodynamic Noise Prediction for Long-Span Bodies,” AIAA Paper 2006-2573, 2006.

47 FfowcsWilliams, J. E. and Hawkings, D. L., "Sound Generation by Turbulence and Surfaces in Arbitrary Motion," Philosophical Transactions of the Royal Society, Vol. A264, No. 1151, 1969, pp. 321-342.

48 Dobrzynski, W. and Pott-Pollenske, M., "Slat Noise Source Studies for Farfield Noise Prediction," AIAA Paper 2001-2158, 2001.

49 Roger, M. and Perennes, S., "Low-Frequency Noise Sources in Two-Dimensional High-Lift Devices," AIAA Paper 2000-1972, 2000.

50 Pott-Pollenske, M., Alvarez-Gonzalez, J., and Dobrzynski, W., "Effect of Slat Gap/Overlap on Farfield Radiated Noise," AIAA Paper 2003-3228, 2003.

51 Terracol, M., Manoha, E., and Lemoine, E., "Investigation of the Unsteady Flow and Noise Sources Generation in a Slat Cove: Hybrid Zonal RANS/LES Simulation and Dedicated Experiment," AIAA Paper 2011-3203, 2011.

52 Deck, S. and Laraufie, R., "Numerical Investigation of the Flow Dynamics Past a Three-element Aerofoil," Journal of Fluid Mechanics, Vol. 732, 2013, pp. 401-444.

53 Rossiter, J., "Wind-Tunnel Experiments on the Flow over Rectangular Cavities at Subsonic and Transonic Speeds," Aeronautical Research Council Reports and Memoranda 3438, 1966.

54 LEISA slat-noise configuration

URL: https://info.aiaa.org/tac/ASG/FDTC/DG/BECAN_files_BANCII_category6 [cited 15 April 2015].

43

American Institute of Aeronautics and Astronautics 